Paula Hanna Valdujo

\title{
Diversidade e distribuição de anfíbios no \\ Cerrado: o papel dos fatores históricos e dos gradientes ambientais
}

São Paulo

2011 
Paula Hanna Valdujo

\title{
Diversidade e distribuição de anfíbios no Cerrado: o papel dos fatores históricos e dos gradientes ambientais
}

\author{
Diversity and distribution of anurans in \\ Brazilian Cerrado: the role of historical factors \\ and environmental gradients
}

Tese apresentada ao Instituto de Biociências da Universidade de São Paulo, para a obtenção de Título de Doutor em Ciências, na Área de Ecologia.

Orientador: Prof. Marcio Martins

Co-orientadora: Dra. Catherine H. Graham

São Paulo

2011 


\section{Ficha Catalográfica}

\section{Valdujo, Paula Hanna}

Diversidade e distribuição de anfíbios no Cerrado: o papel dos fatores históricos e dos gradientes ambientais

Número de páginas: 125

Tese (Doutorado) - Instituto de Biociências da Universidade de São Paulo. Departamento de Ecologia.

1. Biogeografia 2. Anura 3. Beta diversidade I. Universidade de São Paulo. Instituto de Biociências. Departamento de Ecologia.

\section{Comissão Julgadora:}

$\operatorname{Prof}(a)$. Dr(a).

$\operatorname{Prof}(a) . \operatorname{Dr}(a)$
Prof(a). Dr(a).

Prof(a). Dr(a).

Prof. Dr. Marcio Roberto Costa Martins

Orientador(a) 
Dedico essa tese aos bons professores que me inspiraram ao longo da vida. Este trabalho tem um pouco de cada um deles 


\section{Agradecimentos}

Como tudo começou...

A ideia deste trabalho surgiu em 2006, quando percebi que as bases de dados espaciais de anfíbios eram mais incompletas que as de outros vertebrados, o que comprometia a representatividade do grupo no planejamento para a conservação da biodiversidade no Cerrado. Agradeço à querida Débora Silvano por ter topado na mesma hora a parceria que deu origem a esta tese. Agradeço também ao Cristiano Nogueira, por ter me inspirado com seu belo trabalho com os lagartos do Cerrado e pelos palpites na proposta inicial, e ao Harry Greene, que mesmo achando que eu deveria voltar a estudar serpentes, me encorajou a seguir com minhas ideias.

E assim eu deixei os interflúvios e seus lagartos diurnos para "tentar a sorte" à noite nas poças, nos brejos, nas veredas e nos riachos. Como quase tudo era novidade para mim, contei com a ajuda de diversas pessoas para me entender melhor com o sistema e o grupo de estudo, e aprender o que é importante nessa vida quando se é um sapo! Vários colegas, professores e pesquisadores sempre estiveram dispostos a compartilhar seus conhecimentos a respeito da taxonomia, relações filogenéticas, e história natural das muitas espécies... Muito obrigada Julian, Dill, Carlos Alberto, Chris Strussmann, Reuber, Adrian, Renata Cecília, Vanessa, Felipe Leite, Vanda Ferreira, Antoine. Contei com a gentileza também dos que forneceram dados de ocorrência das espécies quando eu descobri que precisava aumentar muito a precisão dos meus pontos de coleta... Obrigada à Cinthia Brasileiro, Kátia Kopp, Vanessa Verdade, Mauro Teixeira Junior, Carlos Cândido, Cybele Araújo, Henrique Caldeira Costa, Cristiano Nogueira, Felipe Leite, Ricardo Kawashita Ribeiro, Mariana Mira, Reuber Brandão, Adriano Lima Silveira. Ao querido Hebert Ferrarezzi pelas discussões ao vivo e via $m s n$, pelas sugestões, e pelas aulas de biogeografia histórica - você foi fantástico como sempre!

Agradeço ao meu orientador Marcio Martins pela confiança e por darme liberdade, além da oportunidade de colaborar com pessoas que acrescentaram muito a esta tese e à minha forma de trabalhar. Agradeço imensamente por ter aceitado a ideia meio maluca desse projeto, por viabilizar todas as visitas às coleções, apoiar minhas decisões e por estar 
sempre disposto a ajudar no que eu precisei. Mas isso não é uma despedida, pois ainda temos muito o que fazer! Agradeço ao meu assessor da Fapesp por todas as sugestões e pelo entusiasmo que demonstrou com os resultados deste trabalho.

Em sala de aula eu agradeço...

Aos excelentes professores com quem tive a satisfação de aprender em classe. Especialmente ao Glauco, Paulo Inácio, Alê Adalardo, Jean Paul e Miúdo. À equipe e alunos do curso de campo de 2008, de 2009 e... sim, de 2010 ! Virei fã da disciplina e espero poder colaborar com a equipe por muitos e muitos anos! Obrigada por me ensinarem tanto e me darem a oportunidade de repassar um pouquinho do conhecimento para os que vieram depois. Ter sido aluna desse curso me fez crescer muito, ter sido monitora foi uma experiência incrível! A recompensa vale cada gota de suor e de chuva... Agradecimentos especiais aos companheiros de monitoria Billy, Camila Castanho, Marie, Marcel e Murilo.

No campo...

Tive a oportunidade de trabalhar no campo em 11 localidades diferentes e aprender com pessoas fantásticas. Meu super-mega-max especial agradecimento ao Recoder, que foi literalmente aos quatro cantos do Cerrado comigo - do Paraná ao Maranhão, e do Mato Grosso à Bahia. Aos meus companheiros de campo, fossem eles assistentes, parceiros ou chefes: Mauro, Agustin, Mara, Renan, Gabriel Costa, Cristiano, Rê Moretti, KK, Johnny, Cardoso, Mari Mira, Xexa, Santos, Miguel Rodrigues, Tamí, Magno e Bianca. Agradeço pela oportunidade de trabalhar, aprender e conviver com vocês, pelo companheirismo, pelas memoráveis noites nos brejos, lagoas e riachos, e por me ajudarem a coletar os dados mais suados, molhados e divertidos deste trabalho.

Pela fase de estudos nas coleções, eu agradeço...

Aos curadores que prontamente permitiram-me acessar os espécimes e registros de campo: Hussam Zaher, Guarino Colli, Marcos Carvalho, Rogério Bastos, Felipe Toledo, Célio Haddad, Luciana Nascimento, Denise Rossa-Feres, Franco Leandro de Souza, José P. Pombal Junior, Ulisses Caramaschi, e Ulisses Galatti. Também aos alunos, estagiários e técnicos por 
facilitarem muito meu trabalho na coleção, especialmente à querida Carol Mello, que foi parceira incrível no meu desafio de examinar uma quantidade insana de espécimes no MZUSP. Agradecimentos especiais também à Mari Gomes, estagiária do projeto "Conservação dos Anfíbios do Cerrado", pela paciência infinita em passar todos os registros das planilhas Excel para o banco de dados do Biota, e ao Robert Cowell por estar sempre disponível para converter o arquivo entre as versões PC e Mac do Biota.

E chega o momento das análises...

Agradeço ao Miltinho e aos colegas da CGZAM-IBAMA, Zuca, Jailton e Guilherme, por tudo o que me ensinaram na arte dos sistemas de informação geográfica. Agradeço ao Paulo Inácio e ao Alê por me iniciarem no mundo maravilhoso do "R". Mais uma vez, ao Paulo Inácio pelos ensinamentos sobre modelagem estatística - sim, foi um turning point na minha vida acadêmica, pode acreditar!

So I decided to go abroad and learn some more...

Ana Carnaval was super-helpful with the arrangements for my shortterm visit in the US. Ana contacted Dr. Catherine Graham in Stony Brook and also invited me to go to Berkeley for a few days. The partnership with these two amazing researchers was just perfect, and I am glad to share plans for the next couple years with them. After Ana's recommendation, Dr. Catherine Graham kindly accepted me as a visiting student, and was extremely helpful and supportive during the period that I was in the US. I also thank all the staff of the Department of Ecology and Evolution, Stony Brook University, especially Iris Roth, Martha Nolan, Dona DiGiovanni, and Fumio Aoki. They were super-helpful with paperwork, mail and computer stuff. I thank Juan Parra for helping me to figure how to run the GDM commands. Juan, I would have never made it without you, thank you so much! I thank Jessie for revising my writing so many times, taking me to school and back home on rainy-snowy-freaking-cold-days, and for being such an amazing friend to hang out with! Thank you for making my days so much nicer, Jessie! I thank my friends Phil, Dan, Sarah, Lee, Boris, Mayte, Jorge, Norah, and Pedro Peloso for their support, rides, and for being around!

E na hora de escrever... 
Tive a felicidade de contar com o apoio de pessoas fantásticas que mudaram dramaticamente o meu estilo de escrever. A primeira emoção foi descobrir que existe técnica na produção de uma introdução... Antes tarde do que nunca... Obrigada mestre Glauco por mais essa! Também vou me lembrar para sempre da Catherine dizendo "Paula, you have to pretend you are telling a story! Let's start this text with 'once upon a time..."' - acho que funcionou!

E muitas vezes ao longo dos últimos quatro anos eu agradeço...

À minha parceiríssima de projeto Débora, por compartilhar todas as etapas e quase todos os momentos desse processo. Não preciso dizer que não teria a menor chance de chegar a essa base de dados gigante super organizada sem você, né? Pela paciência em todas as vezes que resolvi alterar as minhas perguntas e os meus capítulos, e por não ter me jogado na janela quando eu mudava de idéia... Pela oportunidade de colaborar na coordenação do Plano de Ação e por sempre me incluir em tudo. Adorei compartilhar todos esses trabalhos com você. Que venham os próximos!

Aos labvertianos atuais, pretéritos e fósseis: Lourinha, Mandi, Irina, Cinthia, Ricardo e Crinog pelas sugestões, revisões e pela companhia. Especialmente à minha little sis Má Gaiarsa, por todas as revisões de manuscritos, ensaios de disciplina, apresentações... Por fazer milhares de coisas chatas por mim quando eu não estava em SP, como imprimir e levar meus relatórios pra Fapesp, buscar extrato da reserva técnica no banco e histórico escolar na secretaria...

E por falar em secretaria, agradeço...

Ao Departamento de Ecologia da USP e seus funcionários Dalva, Bernadete, Socorro, Luís, Welington, e aos funcionários da seção de pós Cynthia, Erika, Helder, Roberta e Vera. Aos coordenadores da Pós Astrid e Paulo Inácio por todo o trabalho que tiveram e tem para manter o programa.

À Fapesp pela concessão da bolsa (processo 2007/51956-6) e pelo auxílio concedido ao meu orientador (06/58011-4), que possibilitou todas as visitas às coleções. À Fundação Boticário de Proteção à Natureza pelo financiamento parcial da expedição ao Jalapão (TO), algumas das visitas às coleções e todas as atividades relacionadas à construção do banco de dados (projetos 0747_20071 e 0798_20082). À Conservação Internacional por 
parte do financiamento das expedições ao Jalapão e ao Parque Nacional da Chapada das Mesas (MA; projetos CP FY 08/017 e CP FY 08/018). À Pequi Pesquisa e Conservação do Cerrado pela gestão dos projetos e pela L-200. À Natural Grasslands Conservancy pelo small grant que ajudou a viabilizar a expedição para o Oeste da Bahia.

Por fim, mas não menos importante, agradeço...

Aos amigos que estiveram por perto (ou não tão perto) nestes quatro anos, e que de alguma forma tornaram meus dias de doutorado mais fáceis e divertidos. Obrigada à Rê Faria, Moki, Laureta, Sassá Outeda Jorge, Leandro Tambosi, Mari Vidal, Alê Igari, Mari Zatz, Gustavo Alemão, Isa Schmidt, Bel Figueiredo, Mari Napolitano. Agradecimentos especialíssimos à Rê Moretti, que é tão minha amiga que não quis me deixar sozinha e foi pra NY também! Mina, você foi 100\%: fez música pra mim (haha!), me fez rir, me sugeriu seguir formiguinha, me deu conselho quando eu precisei, beliscão quando eu mereci, e ainda me ajudou na revisão do texto da tese.

Aos meus pais, Heloisa e Wagner, pelo carinho, pelo apoio incondicional e por serem meu "hd externo" para armazenar as informações básicas sobre a vida real que a minha cabeça teima em não guardar... Aos meus irmãos, Cássio e Fernando (ordem alfabética), pelo carinho e por aceitarem de bom grado o papel de help-desk sempre que necessário. Aos meus avós, tios e primos por todo apoio e todo o carinho. À Inês, Yago e Mariana, em nome da (cada vez maior) família Zatz, pelo carinho e por estarem sempre dispostos a ajudar com o que eu precisasse. Agradeço ao Leandro por me mostrar como é bom compartilhar, e por fazer de mim uma pessoa melhor e muito mais feliz. Vocês todos sempre foram e sempre serão o meus exemplos! 


\section{Índice}

$\begin{array}{ll}\text { Resumo } & 01\end{array}$

$\begin{array}{ll}\text { Abstract } & 02\end{array}$

Introdução Geral 03

Capítulo 1. Anuran species composition and distribution patterns in $\begin{array}{ll}\text { Brazilian Cerrado, a Neotropical hotspot } & 14\end{array}$

$\begin{array}{ll}\text { Abstract } & 15\end{array}$

1.1. Introduction 15

$\begin{array}{ll}\text { 1.2. Methods } & 17\end{array}$

1.3. Results 19

1.4. Discussion 25

1.5. References 29

1.5. Appendix 34

Capítulo 2. A historical perspective on the distribution of anuran amphibians in Brazilian Cerrado 40

Abstract 41

1.1. Introduction 41

1.2. Methods 45

1.3. Results 49

1.4. Discussion 53

1.5. References 57

Capítulo 3. Environmental and historical correlates of anuran $\begin{array}{ll}\text { betadiversity in Brazilian Cerrado } & 60\end{array}$

$\begin{array}{ll}\text { Abstract } & 61\end{array}$

1.1. Introduction 61

1.2. Methods 64

1.3. Results 70

1.4. Discussion 76

1.5. References 81 
Discussão Geral e Conclusões

Anexos e Apêndices 


\section{Resumo}

A integração de fatores contemporâneos locais e procesos biogeográficos fornece uma visão ampla e promissora a respeito da diversidade de espécies e seus padrões de diversidade. Utilizando ferramentas recentes para análises espaciais, eu integro fatores históricos e contemporâneos para analisar a distribuição e beta diversidade espécies de anuros do Cerrado. Forneço informações atualizadas a respeito da composição e distribuição das espécies de anuros no Cerrado, com base em um extensivo levantamento em coleções zoológicas e estudos de campo. Analiso a distribuição e beta diversidade em um contexto histórico, enfocando as relações do Cerrado com seus domínios vizinhos, e suas condições ambientais. Registrei 204 espécies de anuros no Cerrado, das quais 50\% são endêmicas.

Espécies que ocorrem no Cerrado e mais um domínio apresentam alta estruturação espacial, na qual espécies amazônicas estão restritas à porção noroeste e as espécies atlânticas estão restritas à porção sudeste do Cerrado. Registrei espécies endêmicas em quase todas as localidades e em todas as regiões, enquanto espécies de distribuição restrita ocorrem apenas em regiões montanhosas no centro, sudeste e sudoeste do Cerrado. Gêneros originado na Mata Atlântica e Amazonia estão distribuídos em um padrão de "tabuleiro de xadrez" dentro do Cerrado, e co-ocorrem menos do que seria esperado ao acaso. Essa estruturação espacial no Cerrado é influenciada pela ação combinada das condições ambientais e restrições históricas: gêneros atlânticos estão predominantemente distribuídos em

áreas de montanha, com baixa precipitação e próximas ao limite com a Mata Atlântica, enquanto gêneros amazônicos estão distribuídos em vales mais próximos ao limite com a amazônia. Da mesma forma, os padrões de beta diversidade no Cerrado parece estar fortemente influenciados pelos gradientes ambientais, uma vez que metade da dissimilaridade na composição de espécies entre taxocenoses foi explicada por estes preditores. Espécies endêmica responderam às condições ambientais regionais de forma mais intensa que todas as espécies em conjunto. A resposta das espécies endêmicas parece estar relacionada na similaridade do ambiente em que ocorrem em relação aos domínios vizinhos, uma vez que suas espécies-irmãs estão principalmente distribuídas por estas regiões. Meus resultados reforçam a importância de se considerar a história biogeográfica das linhagens nas análises dos padrões regionais de disversidade. Demonstro também que a heterogeneidade na distribuição das espécies de anuros pode ter uma base histórica, que interage com restrições atuais, como o clima, disponibilidade de habitat e interações ecológicas na montagem de comunidades. 


\section{Abstract}

Integrating local contemporary factors and biogeographic processes allows a promising and broad view on species diversity and distribution patterns. Building on the development of new tools for spatial analysis, I integrate historical and contemporary factors that may explain species distribution and beta diversity patterns of anuran amphibians in Brazilian Cerrado. Especifically, I update information about species composition and distribution of anurans in the Cerrado, based on an extensive search in zoological collections and fieldwork. I analyze distribution and beta diversity in a historical framework, focusing on the relationship of the Cerrado with its adjoining domains, and its environmental conditions. I found 204 anuran species occurring within the Cerrado, from which $50 \%$ are endemics. Species occurring in the Cerrado and one more domain present a highly structured spatial pattern, in which Amazonian species are restricted to the northwestern part of the Cerrado and Atlantic species are restricted to the southeastern part. I found Cerrado endemics in most of the localities, in all regions, whereas narrow endemics are restricted to mountain ranges in central, southeastern, and southwestern Cerrado. Cerrado anuran genera originated in Atlantic Forest and Amazon are distributed in a checkerboard pattern, and co-occur less than it would be expected by chance. This spatial structure within the Cerrado is influenced by the interplay of environmental conditions and historical constrains: Atlantic genera are mostly distributed in mountainous and upland areas, with low precipitation and closer to the boundaries of Atlantic Forest, whereas Amazonian genera are distributed in valleys closer to the boundaries of the Amazon. Similarly, patterns of beta diversity in the Brazilian Cerrado appear to be strongly influenced by the environmental gradients, since half of the dissimilarity in species composition was explained by these predictors. Endemic species responded to regional environmental conditions stronger than all species. Endemics may be responding to environment based on how similar they are to the conditions of adjoining phytogeographical domains, since their sister-species are mostly distributed in these regions. My results reinforce the importance of taking biogeographical history into account when analyzing spatial patterns of species diversity at a regional scale. I also show that the heterogeneity in anuran distribution in the Cerrado may have a historical basis, which interacts with present-day constraints, such as climate, habitat availability and ecological interactions, to shape local and regional assemblages. 


\section{Introdução Geral}

Análises de processos ecológicos que geram os padrões de distribuição de grupos monofiléticos em uma dada região são úteis para o entendimento dos condicionantes da diversidade regional de espécies e de seus padrões de distribuição (Ricklefs \& Schluter 1993; Wiens \& Donoghue 2004; Cavender-Bares et al. 2009). Para isso, é necessário que sejam aumentadas tanto as escalas espaciais e temporais quanto filogenéticas, o que permite a interpretação dos fatores locais contemporâneos, dos processos biogeográficos de longo prazo e das conexões entre ambos. Entende-se por fatores locais contemporâneos as relações das espécies com o ambiente, incluindo as condições climáticas locais e a disponibilidade de microhabitats, bem como as relações com outras espécies, sejam elas competidores, presas ou predadores. Já os processos biogeográficos referem-se à história das linhagens às quais as espécies pertencem, bem como a seus eventos de dispersão, especiação e extinção ao longo do tempo. Embasando-se no desenvolvimento de novas ferramentas para análises espaciais, esse estudo integra fatores históricos e contemporâneos para explicar a distribuição de espécies e os padrões de beta-diversidade de anfíbios anuros no Cerrado brasileiro.

Os organismos diferem quanto a seus limites de tolerância ecofisiológica e requisitos relacionados ao habitat, o que resulta em limitações em suas distribuições (Schluter \& Ricklefs 1993). Informações a respeito das condições ambientais ao longo de toda a extensão da distribuição de uma espécie e até mesmo de suas espécies-irmãs podem ser úteis para compreender seus requisitos e limites ecofisiológicos. Conhecendo essas características, podem-se compreender os motivos pelos quais a espécie ocorre em determinadas áreas e não ocorre em outras. Essas informações são úteis para interpretar as dissimilaridades na composição de espécies entre comunidades ao longo de gradientes ambientais, nos quais diferentes espécies ficam limitadas a partes distintas do gradiente (Ferrier et al. 2007; Buckley \& Jetz 2008; Graham \& Fine 2008).

Além dos fatores contemporâneos, representados pelas respostas ecofisiológicas e comportamentais às condições ambientais, a distribuição 
das espécies é também influenciada por fatores ligados à história biogeográfica de suas linhagens (Buckley \& Jetz 2007, 2008). As restrições históricas estão relacionadas ao fato das espécies compartilharem características morfológicas e ecofisiológicas com outras espécies filogeneticamente relacionadas a elas, como resultado de sua ancestralidade comum (Felsenstein 1985; Brooks \& McLennan 1991). Dessa forma, esperase que espécies-irmãs ocupem ambientes de características climáticas e topográficas semelhantes, conservando o nicho ambiental de seus ancestrais (Wiens \& Graham 2005). Ainda, a forma pela qual as espécies respondem aos gradientes ambientais depende dos padrões de especiação e extinção que determinam o pool regional de espécies (Ricklefs 2004; Harrison \& Cornell 2007).

A ação combinada das restrições ambientais e históricas explica a distribuição global da riqueza de anfíbios (Buckley \& Jetz 2007) e aves (Hawkins et al. 2003). Entretanto, pelo fato dos contingentes históricos terem sido avaliados indiretamente, representados pelas zonas biogeográficas, 0 papel seu papel confunde-se aos fatores ambientais, dificultando a elaboração de predições a respeito da variação na diversidade de espécies em diferentes partes do mundo (Whittaker \& Field 2000). Especificamente no caso dos anfíbios, a combinação entre disponibilidade de energia e de água foi o fator ambiental mais importante para a determinação dos padrões de riqueza de espécies (Hawkins et al. 2003). Além disso, este fator combinado com a classificação em províncias biogeográficas, forneceu a melhor explicação possível para os gradientes de riqueza (Buckley \& Jetz 2007), evidenciando a importância dos fatores históricos e ambientais. A dissimilaridade entre taxocenoses de anfíbios em escala global aumenta com a dissimilaridade ambiental, mas não é dependente dela, pois pares de localidades com condições ambientais semelhantes podem apresentar alta dissimilaridade, reforçando o papel dos condicionantes históricos (Buckley \& Jetz 2008).

Na América do Sul, a riqueza e o endemismo de anfíbios apresentam forte estruturação espacial (IUCN 2010). A maior parte da riqueza concentrase nas florestas tropicais, especialmente na Mata Atlântica e na Amazônia (Buckley \& Jetz 2007; figura 1A). Este padrão é consistente com a hipótese 
de que a pluviosidade é um importante fator determinante dos padrões de riqueza. Por outro lado, o endemismo de anfíbios é mais alto nas regiões de topografia mais acentuada, tais como a cordilheira dos Andes e a Mata Atlântica, onde as espécies estão sujeitas a um maior isolamento (Duellman 1988; figura 1B). A alta riqueza de espécies endêmicas e sua concentração em determinadas áreas resulta em alta beta-diversidade nessas áreas, considerando aqui beta-diversidade como a dissimilaridade entre células adjacentes em um grid de $100 \times 100 \mathrm{~km}$ sobre o continente americano (McKnight et al. 2007).
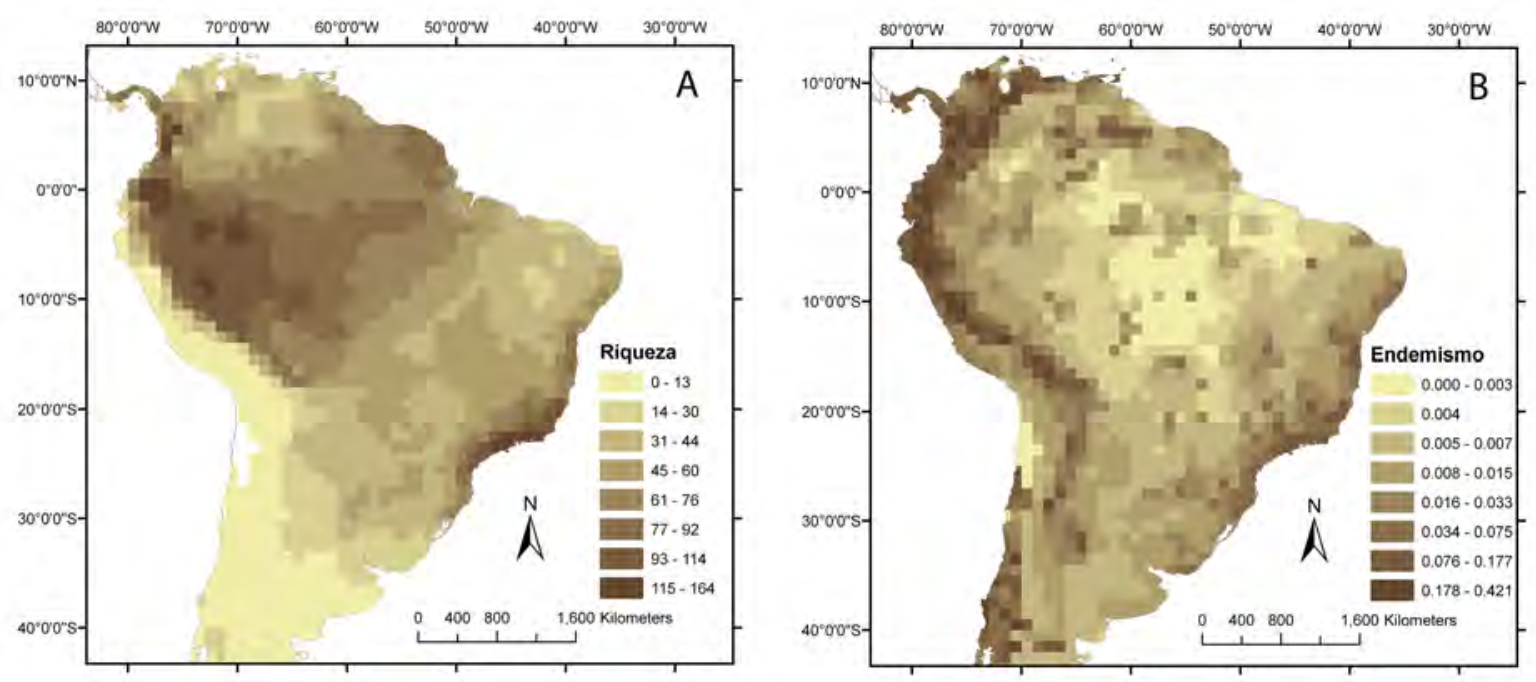

Figura 1: Valores de riqueza $(A)$ e endemismo ponderado $(B)$ por célula de $1^{\circ}$ por $1^{\circ}$ calculados a partir dos mapas disponíveis em IUCN (2010). O endemismo ponderado é calculado como o somatório do número de espécies que ocorre em cada célula do gride ponderadas pelo inverso do tamanho de sua distribuição. Para eliminar o efeito da riqueza, o valor obtido é dividido pelo número de espécies em cada célula (Crisp et al. 2001).

Os anfíbios tem distribuição mais restrita em relação a outros vertebrados terrestres por serem mais limitados pelas condições ambientais (Feder \& Burggren 1992; Buckley \& Jetz 2007) e por apresentarem capacidade de dispersão limitada (Smith \& Green 2005). Diversas adaptações morfológicas, fisiológicas e comportamentais, combinadas com a 
grande diversidade de modos reprodutivos, permitem que os anfíbios ocorram em diversos tipos de ambientes (Duellman \& Trueb 1986; Duellman 1988; Haddad \& Prado 2005). A região Neotropical, especialmente nas florestas pluviais, apresenta a maior diversidade de anuros no mundo (IUCN 2010). Essa diversidade diminui das zonas mais úmidas para as mais secas e das zonas mais baixas para as mais altas, sendo também influenciadas pela história dos grupos filogenéticos (Duellman 1988; Buckley \& Jetz 2007). As espécies de anuros que ocorrem no Neotrópico podem ser divididas em dois principais grupos ecológicos: um grande grupo de espécies restritas a florestas úmidas e um grupo menor de espécies típicas de formações abertas, que ocorrem nos domínios fitogeográficos do Cerrado, do Chaco e da Caatinga, bem como em áreas de vegetação aberta dos demais domínios (Heyer 1988).

A anurofauna do Cerrado compreende um grande número de espécies endêmicas do domínio (Bastos 2007), espécies compartilhadas com domínios vizinhos e algumas espécies amplamente distribuídas (Colli et al. 2002). A distribuição da riqueza total de espécies e da riqueza de espécies endêmicas é heterogênea dentro do bioma (Diniz-Filho et al. 2004; DinizFilho et al. 2007; Diniz-Filho et al. 2008) sendo que os dois índices apresentam maiores valores na porção sudeste do Cerrado. Apesar do esforço de amostragem ser historicamente mais intenso na porção sul do Cerrado (Diniz-Filho et al. 2005; Diniz-Filho et al. 2008), esse viés não pode ser reconhecido como o principal fator determinante da heterogeneidade do bioma, uma vez que localidades bem amostradas em diferentes partes do Cerrado diferem substancialmente em composição e riqueza (Eterovick \& Sazima 2000; Brasileiro et al. 2005; Vaz-Silva et al. 2007; Brasileiro et al. 2008; Valdujo et al. 2009). Além da heterogeneidade espacial e ambiental do Cerrado (Silva et al. 2006), diferenças em sua composição de espécies devem ter sido favorecidas pelo contato com quatro grandes domínios fitogeográficos da América do Sul: Amazônia, Mata Atlântica, Caatinga e Chaco (Joly et al. 1999). Historicamente, outra fonte de diversidade são as espécies e linhagens típicas dos domínios vizinhos que podem ter sido recrutadas destes domínios e evoluído in situ, como já foi descrito para quatro linhagens de plantas (Simon et al. 2009). 
Vários aspectos fazem do Cerrado um sistema-modelo de interesse para estudos biogeográficos. Em primeiro lugar, sua posição central, estabelecendo contato com cinco outros domínios, determina um alto potencial para trocas de espécies ao longo da história biogeográfica das linhagens, favorecendo a alta diversidade de espécies e de linhagens na escala regional. A heterogeneidade ambiental dentro do bioma também oferece habitats e condições diversas para espécies com diferentes requisitos ecofisiológicos. As paisagens no Cerrado são dominadas por formações savânicas e campestres, porém matas de galeria e manchas de florestas deciduais e semideciduais são frequentes e espalhadas pelo domínio, e representam uma importante conexão com os domínios florestais vizinhos, pelo menos para grupos de plantas (Oliveira-Filho \& Ratter 1995) e pequenos mamíferos (Costa 2003). Apesar do papel das manchas de florestas para a distribuição dos anuros nunca ter sido sistematicamente investigado, sabe-se que algumas espécies típicas de outros domínios, tanto abertos quanto florestais, podem ocorrer marginalmente ou profundamente no Cerrado, especialmente nas florestas de galeria (Colli et al. 2002). Por outro lado, os interflúvios planálticos tendem a funcionar como filtros ambientais, devido à menor hetetogeneidade e umidade dos ambientes.

A história do Cerrado e sua biota ainda está longe de ser revelada. Estudos paleoflorísticos (Cole 1986; Romero 1993) indicaram que o Cerrado é bastante antigo e originou-se a partir de um tipo de vegetação de clima mais seco e frio que ocorria na porção sul da América do Sul no Cretáceo Superior, após a separação entre a América do Sul e a África. Esta hipótese contradiz a visão de Rizzini (1976) de que as áreas atualmente cobertas pelo Cerrado originaram-se de formações florestais que foram se tornando mais secas e abertas por meio de processos naturais, como o fogo. A hipótese de Cole (1986) foi corroborada por dois estudos realizados com fauna de formações abertas. Pascual \& Jareziguar (1990) demonstraram que durante o Cenozóico a maior parte da fauna de mamíferos da América do Sul era adaptada à savana, indicando que esse tipo de ambiente já cobria a porção central do continente. As áreas abertas tiveram grande importância na diversificação da avifauna do Cerrado, uma vez que a grande maioria das espécies consideradas "paleoendêmicas" são habitantes de formações 
campestres ou savânicas (Silva \& Bates 2002). Por outro lado, Simon et al. (2009) demonstram que diversas espécies de plantas endêmicas do Cerrado com adaptações ao fogo, pertencentes a quatro irradiações independentes, são componentes recentes dentro das linhagens, e apresentam origens em diferentes domínios fitogeográficos. Estudos que combinem hipóteses filogenéticas e análises espaciais de espécies endêmicas podem ajudar a desvendar o papel das múltiplas dispersões a partir de domínios vizinhos e da diversificação in situ para a montagem das taxocenoses no Cerrado.

A dinâmica de dispersão dos organismos e a especiação estão ligadas também à história da distribuição dos domínios fitogeográficos ao longo dos ciclos de flutuação climática, que provocaram expansões e contrações dos domínios florestais na América do Sul. Modelos de paleoclima, validados por registros palinológicos, indicam que a zona de transição do Cerrado com a Mata Atlântica sofreu deslocamento para o interior durante os períodos mais úmidos, que ocorreram no Holoceno, e para o litoral, com severa fragmantação, durante o Pleistoceno, quando houve períodos mais frios e secos. (Carnaval \& Moritz 2008). De maneira análoga, registros palinológicos e simulações de cobertura vegetal baseadas em fatores climáticos indicam que o mesmo processo de expansão e contração da floresta ocorreu na transição do Cerrado com a Amazônia (Mayle et al. 2004), favorecendo a dispersão e subsequente isolamento de organismos entre os dois domínios. Contudo, muitas espécies tem história de diversificação anterior ao Pleistoceno e portanto não explicada pelas flutuações climáticas dos últimos 20 mil anos. Estudos moleculares indicam que tanto eventos ocorridos no Terciário quanto no Quaternário foram importantes para a diversificação das espécies de anfíbios no Neotrópico (Brunes et al. 2010)

Além da história da vegetação da região hoje coberta pelo Cerrado e da presença tanto de espécies antigas quanto recentes, a dinâmica da topografia tem um papel potencialmente importante sobre a diversidade e a distribuição das espécies no Cerrado. Apesar de haver pouca informação disponível a respeito dos processos orogenéticos localizados ocorridos na América do Sul, sabe-se que o Terciário foi, de uma maneira geral, um período marcado por eventos de soerguimento em várias regiões (Petri \& Fúlfaro 1983). Pode ser citada como exemplo a paleocordilheira do 
Espinhaço, aplainada entre o Cretáceo e o Eoceno e re-entalhada por um soerguimento no decorrer do Cenozóico (Ab'Saber 2000). Assim, modificações pós-cretácicas ocorridas no relevo da região central do Brasil (Ab'Saber 2000) podem ter isolado populações de espécies ancestrais, que teriam dado origem às espécies endêmicas observadas atualmente. Deste modo, espera-se que a distribuição das espécies de anuros do Cerrado responda não só ao gradiente climático, como também à topografia e às relações com os domínios vizinhos.

Nenhum dos estudos publicados tratando de anuros do Cerrado interpretou os padrões de diversidade em um contexto histórico, incorporando informações a respeito da distribuição das linhagens e grupos-irmãos, e enfocando sistematicamente a influência dos domínios vizinhos. Este projeto foi proposto no intuito de preencher uma lacuna no conhecimento da anurofauna do Cerrado por meio de inventários exaustivos em coleções zoológicas e em campo. As informações obtidas foram sistematizadas em um banco de dados abrangente e preciso dos pontos de vista taxonômico e geográfico. O principal objetivo consistiu em compreender os condicionantes históricos dos padrões atuais de distribuição das espécies por meio de análises biogeográficas integrativas que também incorporaram aspectos ecológicos e, sempre que possível, temporais.

De forma bastante ampla, eu testei as seguintes hipóteses:

1. A distribuição das linhagens de anuros no Cerrado é explicada pela distância aos domínios vizinhos e pelos gradientes altitudinais;

2. A beta diversidade entre comunidades de anuros no Cerrado é explicada pelas condições ambientais locais e pela história biogeográfica das linhagens.

Esta tese está dividida em três capítulos, escritos na forma de artigos para publicação em revistas internacionais. O primeiro capítulo traz uma descrição detalhada do sistema de estudo, apresentando a lista de espécies atualizada à luz dos novos inventários e descrevendo os padrões de distribuição recorrentes entre diferentes espécies, com um enfoque histórico. O segundo capítulo analisa a distribuição geográfica das diferentes linhagens que compõem a anurofauna do Cerrado, buscando resgatar contingentes históricos que restrinjam a distribuição de espécies endêmicas relacionadas a 
linhagens diversificadas em domínios vizinhos. O terceiro capítulo explora os condicionantes ambientais e históricos dos gradientes de dissimilaridade de espécies entre comunidades no Cerrado. Por fim, são apresentados no Apêndice dois artigos resultantes de levantamentos de campo realizados no âmbito deste projeto, na região de São Desidério, BA e do Jalapão, BA / TO.

\section{Referências}

Ab'Saber, A. N. (2000). Summit surfaces in Brazil. Revista Brasileira de Geociencias 30: 515-516.

Bastos, R. P. (2007). Anfíbios do Cerrado. Herpetologia no Brasil II. L. B. Nascimento and M. E. O. Oliveira. Belo Horizonte, Sociedade Brasileira de Herpetologia: 87-100.

Brasileiro, C. A., R. J. Sawaya, M. C. Kiefer \& M. Martins (2005). Amphibians of an open Cerrado fragment in southeastern Brazil. Biota Neotropica 5: 93-109.

Brasileiro, C. A., E. M. Lucas, H. M. Oyamaguchi, M. T. C. Thomé \& M. Dixo (2008). Anurans, northern Tocantins River Basin, states of Tocantins and Maranhão, Brazil. Check List 4: 185-197.

Brooks, D. \& D. A. McLennan (1991). Phylogeny, Ecology and Behavior - A Research Program in Comparative Biology. Chicago, The University of Chicago Press.

Brunes, T., F. Sequeira, C. F. B. Haddad \& J. Alexandrino (2010). Gene and species trees of a Neotropical group of treefrogs: genetic diversification in the Brazillian Atlantic Forest and the origin of a polyploid species. Molecular Phylogenetics and Evolution 57: 1120-1133.

Buckley, L. B. \& W. Jetz (2007). Environmental and historical constraints on global patterns of amphibian richness. Proceedings of the Royal Society B: Biological Sciences 274: 1167-1173.

Buckley, L. B. \& W. Jetz (2008). Linking global turnover of species and environments. Proceedings of the National Academy of Sciences 105: $17863-17841$.

Carnaval, A. C. \& C. Moritz (2008). Historical climate modelling predicts patterns of current biodiversity in the Brazilian Atlantic forest. Journal of Biogeography 35: 1187-1201.

Cavender-Bares, J., K. H. Kozak, P. V. A. Fine \& S. W. Kembel (2009). The merging of community ecology and phylogenetic biology. Ecology Letters 12: 693-715.

Cole, M. M. (1986). The Savannas: Biogeography and Geobotany. London, Academic Press.

Colli, G. R., R. P. Bastos \& A. A. Araujo (2002). The Character and Dynamics of the Cerrado Herpetofauna. The Cerrados of Brazil: ecology and natural history of a neotropical savanna. P. S. Oliveira and R. J. Marquis. New York, Columbia University Press: 223-239.

Costa, L. P. (2003). The historical bridge between the Amazon and the Atlantic Forest of Brazil: a study of molecular phylogeography with small mammals. Journal of Biogeography 30: 71-86. 
Crisp, M. D., S. W. Laffan, H. P. Linder \& A. Monro (2001). Endemism in the Australian flora. Journal of Biogeography 28: 183-198.

Diniz-Filho, J. A. F., L. M. Bini, C. M. Vieira, M. C. Souza, R. P. Bastos, D. Brandão \& L. G. Oliveira (2004). Spatial patterns in species richness and priority areas for conservation of anurans in the Cerrado region, Central Brazil. Amphibia-Reptilia 25: 63-75.

Diniz-Filho, J. A. F., L. M. Bini, M. P. Pinto, T. F. L. V. B. Rangel, P. Carvalho, S. L. Vieira \& R. P. Bastos (2007). Conservation biogeography of anurans in Brazilian Cerrado. Biodiversity and Conservation 16: 9971008.

Diniz-Filho, J. A. F., L. M. Bini, C. M. Vieira, D. Blamires, L. Terribile, R. Bastos, G. de Oliveira \& B. Barreto (2008). Spatial patterns of terrestrial vertebrate species richness in the Brazilian Cerrado. Zoological Studies 47: 146-157.

Diniz-Filho, J. A. F., R. P. Bastos, T. F. L. V. B. Rangel, L. M. Bini, P. Carvalho \& R. J. Silva (2005). Macroecological correlates and spatial patterns of anuran description dates in the Brazilian Cerrado. Global Ecology and Biogeography 14: 469-477.

Duellman, W. E. \& L. Trueb (1986). Biology of Amphibians. New York, McGraw-Hill Publishing Company.

Duellman, W. E. (1988). Patterns of species diversity in anuran amphibians in the American tropics. Annals of the Missouri Botanical Garden 75: 79104.

Eterovick, P. C. \& I. Sazima (2000). Structure of an anuran community in a montane meadow in southeastern Brazil: effects of seasonality, habitat, and predation. Amphibia-Reptilia 21: 439-461.

Feder, M. E. \& W. W. Burggren (1992). Environmental Physiology of the Amphibians. Chicago, University of Chicago Press.

Felsenstein, J. (1985). Phylogenies and the comparative method. American Naturalist 125: 1-15.

Ferrier, S., G. Manion, J. Elith \& K. Richardson (2007). Using generalized dissimilarity modelling to analyse and predict patterns of beta diversity in regional biodiversity assessment. Diversity and Distributions 13: 252264.

Graham, C. H. \& P. V. A. Fine (2008). Phylogenetic beta diversity: linking ecological and evolutionary processes across space in time. Ecology Letters. 11: 1265-1277.

Haddad, C. F. B. \& C. P. A. Prado (2005). Reproductive modes in frogs and their unexpected diversity in the Atlantic Forest of Brazil. BioScience 55: 207-217.

Harrison, S. \& H. V. Cornell (2007). Introduction: Merging evolutionary and ecological approaches to understanding geographic gradients in species richness. The American Naturalist 170: S1-S4.

Hawkins, B. A., E. E. Porter \& J. A. F. Diniz-Filho (2003). Productivity and history as predictors of the latitudinal diversity gradient of terrestrial birds. Ecology 84: 1608-1623.

Heyer, W. R. (1988). On frog distribution patterns east of the Andes. Proceedings of a Workshop on Neotropical Distribution Patterns.

IUCN (2010). IUCN Red List of Threatened Species. Version 2010.4. <http://www.iucnredlist.org >. Retrieved 3 January 2011, 2011. 
Joly, C. A., M. P. M. Aidar, C. A. Klink, D. G. McGrath, A. G. Moreira, P. Moutinho, D. C. Nepstad, A. A. Oliveira, A. Pott, M. J. N. Rodal \& E. V. S. B. Sampaio (1999). Evolution of the Brazilian phytogeography classification systems: implications for biodiversity conservation. Ciencia e Cultura 51: 331-348.

Mayle, F. E., D. Beerling, W. D. Gosling \& M. B. Bush (2004). Responses of Amazonian ecosystems to climatic and atmospheric carbon dioxide changes since the last glacial maximum. Philosophical Transactions of the Royal Society of London. Series B: Biological Sciences 359: 499.

McKnight, M. W., P. S. White, R. I. McDonald, J. F. Lamoreux, W. Sechrest, R. S. Ridgely \& S. N. Stuart (2007). Putting beta-diversity on the map: broad-scale congruence and coincidence in the extremes. PLoS Biology. 5: 2424-2432.

Oliveira-Filho, A. T. \& J. A. Ratter (1995). A study of the origin of central Brazilian forests by the analysis of plant species distribution patterns. Edinburgh Journal of Botany 52: 141-194.

Pascual, R. \& E. O. Jaureziguar (1990). Evolving climates and mammal faunas in Cenozoic South American. Journal of Human Evolution 19: 23-60.

Petri, S. \& V. J. Fúlfaro (1983). Geologia do Brasil. São Paulo, Editora da Universidade de São Paulo.

Ricklefs, R. E. \& D. Schluter (1993). Species Diversity in Ecological Communities, Historical and Geographical Perspectives. Chicago, The University of Chicago Press.

Ricklefs, R. E. (2004). A comprehensive framework for global patterns in biodiversity. Ecology Letters 7: 1-15.

Rizzini, C. T. (1976). Tratado de Fitogeografia do Brasil. São Paulo, Editora de Humanismo, Ciê ncia e Tecnologia / Editora da Universidade de São Paulo.

Romero, E. J. (1993). South American paleofloras. Biological Relationships Between Africa and South America. P. Goldblatt. New Haven and London, Yale University Press: 62-85.

Schluter, D. \& R. E. Ricklefs (1993). Convergence and the regional component of species diversity. Species diversity in ecological communities: Historical and geographical perspectives R. E. Ricklefs and D. Schluter. Chicago, The University of Chicago Press: 230-240.

Silva, J. F., M. R. Fariñas, J. M. Felfili \& C. A. Klink (2006). Spatial heterogeneity, land use and conservation in the cerrado region of Brazil. Journal of Biogeography 33: 536-548.

Silva, J. M. C. \& J. M. Bates (2002). Biogeographic patterns and conservation in the South American Cerrado: a tropical savanna hotspot. Bioscience 52: 225-234.

Simon, M. F., R. Grether, L. P. De Queiroz, C. Skema, R. T. Pennington \& C. E. Hughes (2009). Recent assembly of the Cerrado, a neotropical plant diversity hotspot, by in situ evolution of adaptations to fire. Proceedings of the National Academy of Sciences 106: 20359-20364.

Smith, M. A. \& d. M. Green (2005). Dispersal and the metapopulation paradigm in amphibian ecology and conservation: are all amphibian populations metapopulations? Ecography 28: 110-128. 
Valdujo, P. H., R. S. Recoder, M. M. Vasconcellos \& A. S. Portella (2009). Amphibia, Anura, São Desidério, western Bahia uplands, northeastern Brazil. Check List 5: 903-911.

Vaz-Silva, W., A. G. Guedes, P. L. Azevedo-Silva, F. F. Gontijo, R. S. Barbosa, G. R. Aloísio \& F. C. G. Oliveira (2007). Herpetofauna, Espora hydroelectric power plant, state of Goiás, Brazil. Check List 3: 338-345.

Whittaker, R. J. \& R. Field (2000). Tree species richness modelling: an approach of global applicability? Oikos 89: 399-402.

Wiens, J. J. \& M. J. Donoghue (2004). Historical biogeography, ecology and species richness. Trends in Ecology \& Evolution 19: 639-644.

Wiens, J. J. \& C. H. Graham (2005). Niche conservatism: integrating evolution, ecology, and conservation biology. Annual Review of Ecology Evolution and Systematics 36: 519-539. 


\title{
Capítulo 1
}

\author{
Anuran species composition and distribution \\ patterns in Brazilian Cerrado, a Neotropical hotspot
}

Valdujo, P. H., D. L. Silvano, M. Martins \& G. R. Colli. Manuscrito em fase final de preparação que será submetido ao periódico South American Journal of Herpetology 


\title{
Anuran species composition and distribution patterns in Brazilian Cerrado, a Neotropical hotspot
}

\begin{abstract}
Species distribution patterns result from a combined action of multiple factors acting over different spatial and temporal scales. Here we analyze the distribution patterns of amphibians within the Cerrado domain of South America in a historical framework. First, we provide and updated list of species based on an extensive research in zoological collections and fieldwork. We then explore patterns of endemism and the distribution of species occurring both in the Cerrado and each of the adjoining domains, providing directions for future hypotheses test. We found 204 anuran species occurring in localities within the Cerrado. From these, 145 are primarily associated with the Cerrado, and 59 are typical of one of the adjoining domains that marginally occur within the Cerrado. Widespread species account for only $14.5 \%$ of the Cerrado typical species. Species occurring in the Cerrado and one more domain present a highly structured spatial pattern, in which Amazonian species are restricted to the northwestern part of the Cerrado, the Atlantic species are restricted to the southeastern part, the Caatinga species occur on the northeastern part, and the Chaco species occurr in the southwestern part. We found Cerrado endemics in most of the localities, in all regions, whereas narrow endemics are restricted to mountain ranges in central, southeastern, and southwestern Cerrado. The limited distribution of the species that are shared with one of the six adjoining domains, highlights the influence of independent species pools that are more associated with other domains.
\end{abstract}

\section{Introduction}

Studies of the geographical distribution of may provide insights into the history of regional scale species assembly. One promising approach for defining the geographical extent of regional scale studies is the subdivision of global terrestrial surface into ecoregions, which are defined as units containing unique assemblages of natural communities and species (Olson et al. 2001). However, species ranges vary significantly, even between closely related species, and are not necessarily restricted to one ecoregion or 
phytogeographical domain (McDonald et al. 2005). For instance, some species are distributed across entire continents, while others are associated primarily with a specific ecoregion but may slightly extend their range into an adjoining ecoregion. At least three mechanisms are known to generate and maintain wide species ranges: i) wide ecophysiological tolerances, leading to high dispersal ability and the maintenance of genetic and population flows, which prevent isolation and further speciation (Lester et al. 2007); ii) structurally similar habitats (e.g. vegetation physiognomy) occurring in both ecoregions, leading to increased dispersal (Costa 2003); or iii) shared biogeographic history related to the occurrence of forest refugia in more subtropical areas, due to climatic fluctuations in the past (Carnaval \& Moritz 2008). The systematization and description of species distribution patterns in regions with high biodiversity is the first step towards understanding the relative importance of mechanisms by which these biotas are assembled.

The spread of worldwide species databases has supported regional and continental scale analysis of biodiversity (Buckley \& Jetz 2007; Rahbek et al. 2007; Diniz-Filho et al. 2008) and the development of a wide range of tools to describe and analyze geographic patterns (Guo \& Liu 2010; Laffan et al. 2010; Rangel et al. 2010). Therefore, in spite of some caveats and limitations, biodiversity databases assembled from natural history museum data are becoming increasingly useful (Graham et al. 2004). Electronic databases such as GBIF (Global Biodiversity Information Facility) and CRIA's Species Link (Centro de Referência em Informação Ambiental, an information system that integrates data from Brazilian biological collections) facilitate access to occurrence data. However, these databases are not error-proof, and checking the taxonomic identity of specimens and the accuracy of the spatial information through physical examination of specimens and field notes are mandatory (Graham et al. 2004). Continuous collecting efforts, taxonomic revisions and the discovery of new species make the systematization of biodiversity data a highly dynamic activity, especially in the tropics, where knowledge about any taxonomic group is far from complete (Silvano \& Segalla 2005). The increasing amount of information available for regional or continental scale analysis is allowing improved and better-supported conclusions over time. 
Recent studies based on occurrence data of museum specimens from the Brazilian Cerrado (Diniz-Filho et al. 2004; Diniz-Filho et al. 2007; DinizFilho et al. 2008) described heterogeneity in anuran richness and endemism within the domain; with both reaching higher values in the southeastern portion. Although it is recognized that there is a sampling bias towards the southern part of the Cerrado (Diniz-Filho et al. 2005; Diniz-Filho et al. 2008), this bias cannot be acknowledged as the main reason for the heterogeneity in richness and endemism, since well-sampled sites located in different parts of the domain substantially differ in species composition and richness and confirm this trend (Eterovick \& Sazima 2000; Brasileiro et al. 2005; Vaz-Silva et al. 2007; Brasileiro et al. 2008; Valdujo et al. 2009). Apart from the spatial ecological and environmental heterogeneity of the Cerrado (Silva et al. 2006), differences in species composition may be also favored by contacts with four major South American phytogeographic domains: Amazon, Atlantic Forest, Caatinga and Chaco (Joly et al. 1999).

In this paper we describe patterns in anuran species geographic distribution within the Cerrado regarding both endemism and faunal interchange with neighbor phytogeographyc domains, based on an extensive database. We present an updated list of species occurring in the Cerrado, and define which of them are typical Cerrado representatives and which are typical from adjoining domains and occur marginally in the Cerrado. We explore patterns of species composition and distribution from a historical perspective and provide directions for testing more specific hypotheses about historical and contemporary factors influencing species composition and distribution in the Cerrado.

\section{Methods}

We assembled a database on anuran distribution in the Brazilian Cerrado from museum specimen surveys, taxonomic literature records, and field surveys. Museum surveys consisted of two steps: first we assessed collection records either from books or electronic databases, when available. We compiled field data referring to any species collected in a Cerrado locality (Olson et al. 2001), as well as to any Cerrado typical species occurring either inside or outside this domain. We defined as Cerrado typical all species that 
have at least $30 \%$ of its range within the Cerrado or occur in at least $30 \%$ of the Cerrado range. The second step consisted of physical examination of each specimen, comparison with type specimens whenever possible, and with the taxonomic literature, to check species identities. To allow comparisons of specimens housed in different museums, some individuals, especially typespecimens or topotypes, were photographed. Since collections tend to organize their specimens taxonomically, we could make sure that all available specimens from each species considered a priori a "Cerrado typical" were examined. This double-checking procedure enabled us to improve the inference of endemisms. All records gathered from taxonomical literature were included in the database, irrespective of the examination of specimens, since specimens referred in these papers had their identities determined by specialists.

We examined 31,024 specimens collected in Cerrado localities and deposited in the following zoological collections: Museu de Zoologia da Universidade de São Paulo (MZUSP), Museu Nacional do Rio de Janeiro (MNRJ), Coleção Herpetológica da Universidade de Brasília (CHUNB), Coleção Herpetológica da Universidade Federal de Goiás (ZUFG), Museu Paraense Emílio Goeldi (MPEG), Coleção Zoológica da Universidade Federal do Mato Grosso (ZUFMT), Coleção Zoológica da Universidade Federal do Mato Grosso do Sul (ZUFMS), Museu de História Natural Adão Cardoso Universidade Estadual de Campinas (ZUEC), Coleção Célio Haddad Universidade Estadual Paulista, Campus Rio Claro (CFBH), Coleção Herpetológica do Museu de Ciências Naturais da PUC-Minas (MCN), Coleção do Departamento de Zoologia e Botânica (DZSJRP) housed at UNESP, São José do Rio Preto. Since this paper presents only a broad description of distribution patterns, all locations are represented by municipality centroid, irrespective of the exact location where specimens were collected.

In order to fill some of the gaps in Cerrado anuran surveys, from 2007 to 2009 we performed 101 days of fieldwork in eleven Cerrado localities in the states of Mato Grosso, Paraná, Bahia, Tocantins, and Maranhão. We sampled anurans mostly by active search (Crump \& Scott Jr. 1994), and registered any species found visually or by their calls. Since we aimed simply to find as many species as possible, all surveys were made during the rainy 
season, when most species were active and breeding. We sampled all different landscapes that we could detect and no quantification was made. Voucher specimens were deposited in Brazilian zoological collections (MZUSP, CHUNB and CFBH).

We produced distribution maps based on the centroids of municipalities obtained both in the museums and in the field. Species in the genus Pseudopaludicola and the marmoratus group of genus Leptodactylus, except for L. martinezi, were not mapped due to taxonomic problems that would bias the results. We then classified all listed species into one of the following categories: (1) endemic to the Cerrado - any species restricted to the Cerrado (Figure 1); (2) near endemic - any species highly associated to the delimited area that marginally occurs in any other domain; (3) widespread - generalist species that consistently occur in more than two domains, including both open and forest domains; (4) Chaco-Cerrado - species that occur in both Chaco and Cerrado domains; (5) Caatinga-Cerrado - species that occur in both Caatinga and Cerrado domains; (6) diagonal belt of all open domains species that occur in the Caatinga, the Cerrado, and the Chaco domains; (7) Amazon-Cerrado - species that occur in both Amazon and Cerrado domains; (8) Atlantic Forest-Cerrado - species that occur in both Atlantic Forest and Cerrado domains; (9) Southern Brazil-Cerrado - species that occur in both the southern Atlantic Forest (including the Araucaria forest) and the Cerrado domains. Furthermore, the same species were classified as either typical or marginal, representing respectively species that consistently occur in the Cerrado or species typical from other domains that can sporadically occur in the Cerrado, usually related to transition zones. According to our definition, all endemic, near endemic and widespread are typical, whereas species in the other six categories were considered typical only if more than $30 \%$ of its distribution is located within the Cerrado.

\section{Results}

The Cerrado has high species richness, including endemic, widespread, and marginal species. We recorded 204 species, including typical and marginal species, sampled from at least one locality within the Cerrado (see appendix 1). From these, we recognized 145 species as typical, 
because they consistently occur in the Cerrado, and 59 as marginal, since they are primarily associated to other domains and are known only from ecotonal zones within the Cerrado. One hundred and two species are endemic or near endemic. Twelve out of the 145 typical species are undescribed but easily diagnosable taxa found either in visited collections or during fieldwork. A number of examined specimens could not be assigned to any described species, but due to taxonomic problems involving many Neotropical anuran taxa, we adopted a more conservative criterion and did not list them as multiple undescribed taxa.

A high proportion of the listed species is restricted or strongly associated to the Cerrado, while a smaller proportion are widespread. Endemic and near endemic species pooled accounted for $70.3 \%$ of the species that are primarily associated to the Cerrado (typical). Only $14.5 \%$ of typical and $10.8 \%$ of all species are widespread. Most species that occur both in the Cerrado and in one other domain are actually marginal to the Cerrado, especially those occurring in the Atlantic Forest (table 1).

Table 1. Number of anuran species typical and marginal to the Cerrado classified according to the phytogeographical domains where they occur.

\begin{tabular}{lccc}
\hline Distribution pattern & Typical & Marginal & Pooled \\
\hline Endemic & 72 & - & 72 \\
Near endemic & 30 & - & 30 \\
Widespread & 21 & 1 & 22 \\
Chaco / Cerrado & 3 & 7 & 10 \\
Caatinga / Cerrado & 4 & 6 & 10 \\
Diagonal belt of open domains & 5 & 0 & 5 \\
Amazonia / Cerrado & 5 & 9 & 14 \\
Atlantic Forest / Cerrado & 3 & 30 & 33 \\
Southern Brazil / Cerrado & 2 & 6 & 8 \\
\hline Total & 145 & 59 & 204
\end{tabular}


Species occurring in the Cerrado and one other domain (including typical and marginal species) are distributed within this domain in a strongly structured pattern. Species occurring in the Cerrado and the Amazon are concentrated in the western and northern part of the Cerrado (figure 1A), whereas species occurring in the Atlantic Forest and the Cerrado are concentrated in the southern and eastern Cerrado (figure 1B). Caatinga and Chaco species show a strongly non-overlapping distribution within the Cerrado, occurring respectively on the northeastern and southwestern portions (figures $2 \mathrm{~A}$ and $2 \mathrm{~B}$ ). Although the pooled set of endemic species is distributed throughout the entire domain (figure $3 \mathrm{~A}$ ), the narrow endemics (range size up to $60.000 \mathrm{Km}^{2}$ ) are restricted to certain areas; namely the Espinhaço mountain range, Canastra mountain range, Central plateau, upper Araguaia river basin, Guimarães mountain range and Bodoquena mountain range (figure 3B). 

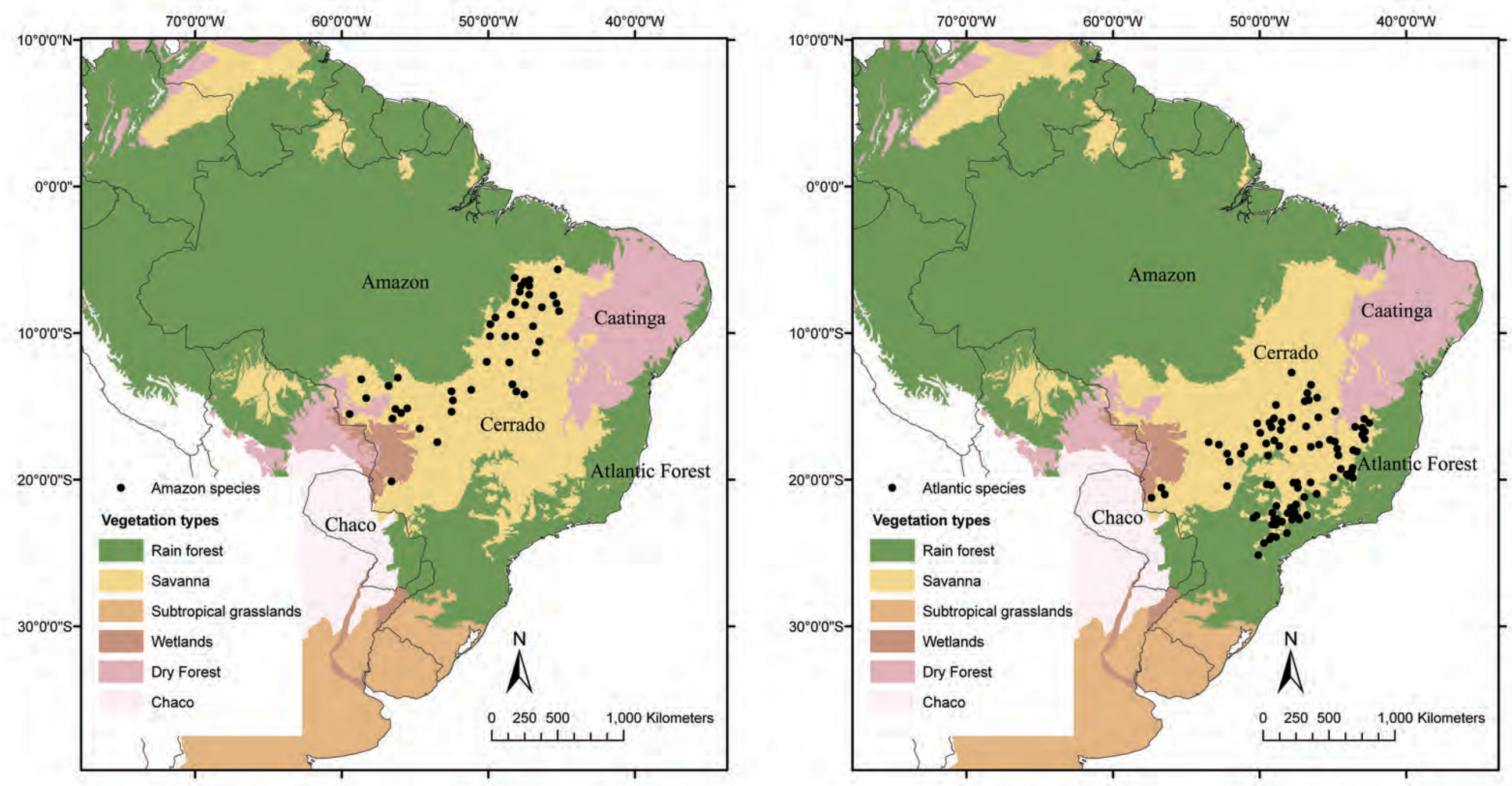

Figure 1. Distribution of anuran species within the Cerrado. A - Distribution of species occurring in the Amazon and the Cerrado; $B$ - distribution of anuran species occurring in the Atlantic Forest and the Cerrado. Colors on the map represent the main vegetation types in South America (modified from Olson et al. 2001) 

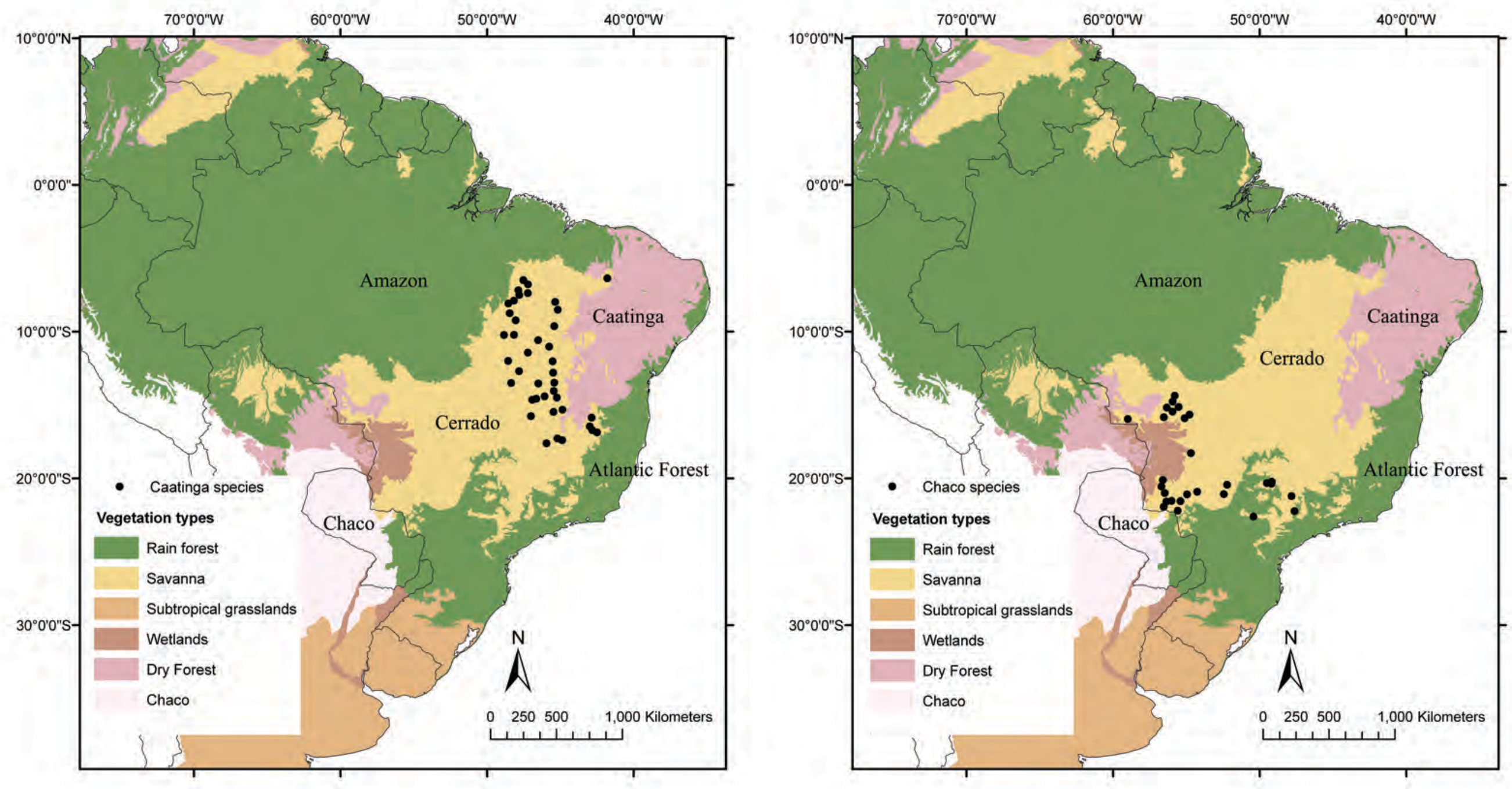

Figure 2. Distribution of anuran species within the Cerrado. A - Distribution of species occurring in the Caatinga and the Cerrado; $B$ - distribution of anuran species occurring in the Chaco and the Cerrado. Colors on the map represent the main vegetation types in South America (modified from Olson et al. 2001) 

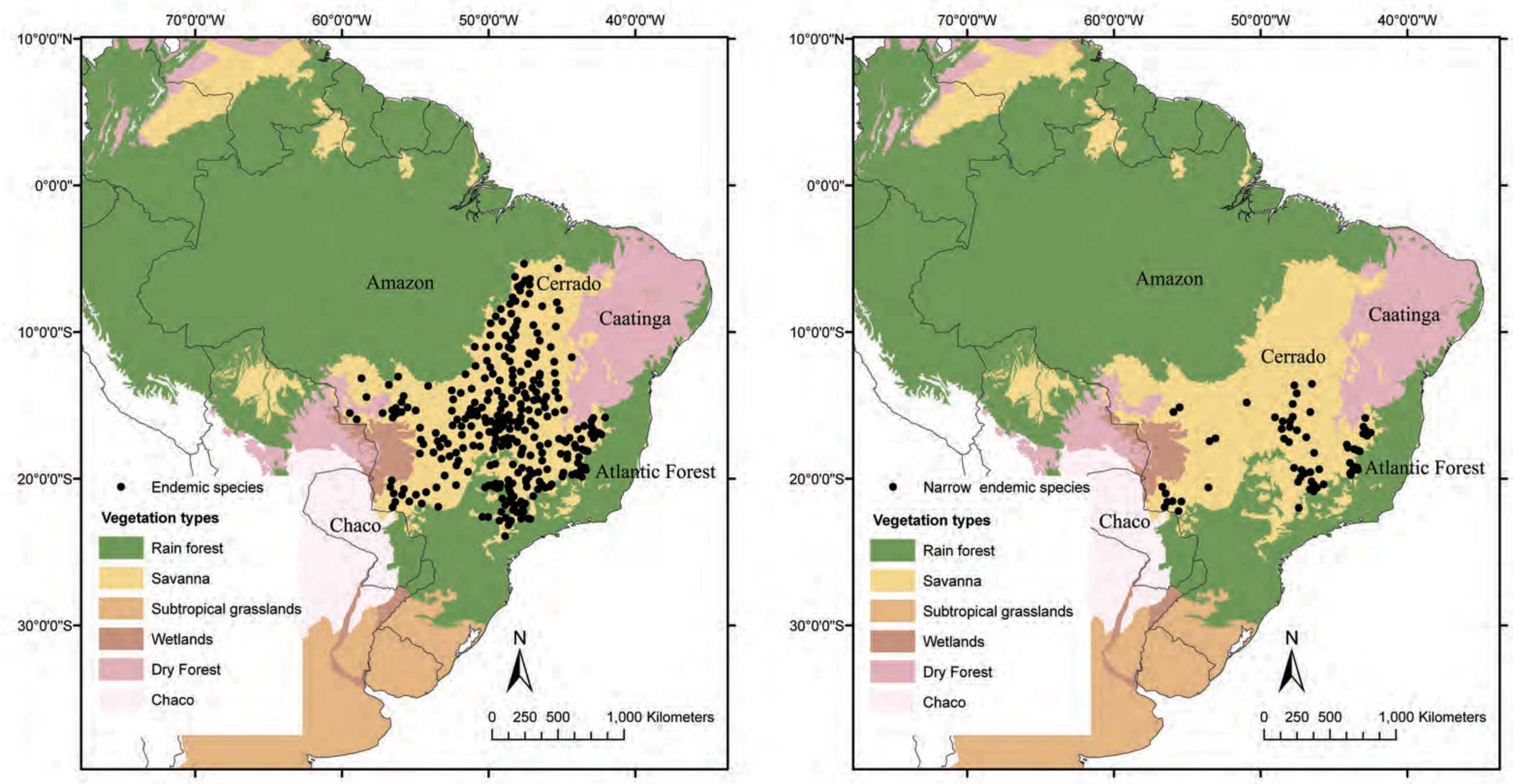

Figure 3. A - Distribution of anuran species endemic to the Cerrado; B - distribution of narrow endemic anuran species within the Cerrado. Colors on the map represent the main vegetation types in South America (modified from Olson et al. 2001) 


\section{Discussion}

The distribution patterns described here suggest that many anurans in the Cerrado likely have overlapping biogeographic histories. Although testing biogeographic hypotheses is beyond the scope of this paper, our results reveal some interesting patterns that are worth future testing within a phylogenetic framework. Cerrado species that are shared with adjoining domains have a remarkable distribution pattern within the Cerrado, in that they are restricted to regions close to their original domains. The limited distribution of these species, shared with one of the six adjoining domains, highlights the influence of independent species pools that are more associated with other domains. This pattern is similar to that described for forest birds in the Cerrado (Silva 1996). Anuran species occurring both in Cerrado and Atlantic Forest do not co-occur with species occurring both in Cerrado and Amazonia. Similarly, anuran species occurring both in Cerrado and Chaco do not co-occur with species occurring both in Cerrado and Caatinga, although there are some other species distributed along all the diagonal belt of open formations in South America. Besides, narrow endemics also have a structured distribution, what is not so evident when all endemics are pooled together. All these geographic restrictions represent a remarkable historical component that contributes to species turnover among Cerrado localities, and seem to be influenced by the low dispersal ability and higher ecophysiological constraints of anurans (Hillman et al. 2009).

The geographic patterns described here are recurrent for species belonging to many unrelated lineages. For instance, the distribution of Chiasmocleis albopunctata (Microhylidae) and Eupemphix nattereri (Leiuperidae) are strongly associated to the limits of the Cerrado. Another pattern is represented by the Amazonian Rhaebo guttatus (Bufonidae) and Osteocephalus taurinus, (Hylidae), which have partially coincident ranges in northeastern Cerrado. A third pattern is represented by the Atlantic Rhinella ornata (Bufonidae) and Hypsiboas albomarginatus (Hylidae), which are restricted to the southeastern margin of the Cerrado. Most of the species occurring in two domains, especially those shared between the Atlantic Forest and the Cerrado, are restricted to the transition areas in the Cerrado. The only Atlantic species that occurs deep within the Cerrado is Aplastodiscus 
perviridis, which seems to show a disjunct distribution. Pseudis bolbodactyla has a unique distribution, since most of its range is located within the Cerrado, but it also occurs deep within Atlantic Forest (Garda \& Cannatella 2007).

\section{Database improvements}

Our results differ in terms of both species composition and rate of endemism from the lists published in previous Cerrado anuran assessments (Colli et al. 2002; Diniz-Filho et al. 2004; Bastos 2007). The use of an extensive database, based on examined specimens, allowed us to access more detailed information covering a greater part of the Cerrado than previous studies. We found no evidence for the occurrence of 18 species listed in at least one of the previous studies. These differences are due to some species being currently under different names, under synonymy, or restricted to Pantanal, Atlantic Forest or Amazon. We added 70 described species not cited by any of the former lists, including recent descriptions and recent records in the Cerrado. These differences are a result of recent collecting efforts in the Cerrado and of the inclusion in our database of specimens obtained from eleven zoological collections, which cover different parts of the domain.

We strongly believe that the total number of species we described does not reflect the actual regional richness in the Cerrado. The recent description of new species in the Cerrado partially corroborates the pattern forecasted by Diniz-Filho et al. (2005), of new species expected to be small-bodied, rangerestricted, and distributed in northern Cerrado. Although species from southern and southeastern Cerrado are still being described (e.g. Caramaschi et al. 2010; Toledo 2010), most recently described species are small bodied and range restricted (e.g. Pugliese et al. 2009; Cardoso \& Pombal-Jr 2010; Nunes et al. 2010). Furthermore, apart from species recently discovered in the field, recent taxonomical revision papers have revealed new species in northern Cerrado (Caramaschi 2010). Although we far surpassed the regional species richness of 160 species forecasted by Diniz-Filho et al. (2005), we believe that the number of recognized species will increase even more in the next few years, given the high number of undescribed species that we found in collections and during fieldwork. 


\section{Centers of Endemism}

The geographic ranges of endemic and near endemic species vary greatly in size and location. Some species are widely distributed within the domain, and also occur in transition areas in adjoining domains (e.g. Eupemphix nattereri and Chiasmocleis albopunctata); others are restricted to a river basin, but are still widely distributed (e.g. Dendropsophus anataliasiasi and Pseudis tocantins in Araguaia / Tocantins river basins); and others are restricted to a specific region (e.g. Rhinella veredas, in the western plateau of Bahia); or restricted to a very specific location (e.g. Physalaemus deimaticus, and Hylodes otavioi, in Serra do Cipó). With a few exceptions of very poorly known species, usually described from long-preserved museum specimens (e.g. Chiasmocleis centralis, Dendropsophus rhea, D. cerradensis, and D. araguaya), restricted range species are mostly distributed in mountainous regions. Several species were described from and are restricted to Serra do Cipó (e.g. Bokermannohyla saxicola and Hypsiboas cipoensis), Serra do Cabral (e.g. Scinax cabralensis), Serra da Canastra (e.g. Bokermannohyla ibitiguara and Scinax maracaya), Chapada dos Veadeiros (e.g. Hypsiboas ericae and Leptodactylus tapiti), and Chapada dos Guimarães (e.g. Phyllomedusa centralis, Pristimantis crepitans).

Eight out of the 30 species considered near endemic to the Cerrado are endemic to the Espinhaço mountain range, a transition zone between both domains. The Espinhaço range is covered by Atlantic Forest on its eastern slope, by Cerrado on its western slope, and by open montane vegetation on its summit; this latter vegetation type is known as campo rupestre and campo de altitude, and is associated with rocky outcrops and sandy soil (Safford 1999). The Espinhaço range is recognized as an area of endemism for plants (Giulietti \& Pirani 1988; Safford 1999; Calió et al. 2008), birds (Silva \& Bates 2002), and more recently for amphibians (Leite et al. 2008). Heyer (1999) stated that many Cerrado endemic species, or species shared with highlands on eastern slopes of the Espinhaço, where enclaves of open vegetation within Atlantic Forest can be found, are related to Atlantic Forest lineages. 


\section{$\underline{\text { Phylogenetic relationships }}$}

Phylogenetic relationships among species occurring in Cerrado are still poorly known, since most Cerrado species were not included in the most recent comprehensive phylogenies (Faivovich et al. 2005; Frost et al. 2006; Grant et al. 2006; Heinicke et al. 2007). A great exception is the recently published phylogeny of Phyllomedusidae (Faivovich et al. 2010), which reveals some interesting distribution patterns, described below. However, for unresolved groups it is possible to interpret phylogenetic relatedness in a preliminary way, since most of the taxonomic arrangements have been corroborated in published phylogenies (Faivovich et al. 2005).

Some Cerrado endemics seem to be more related to taxa distributed in other biomes, such as the Amazon, the Atlantic Forest and the Andes. Species that either belong to Atlantic Forest clades or have their sister-group in the Atlantic Forest include Pseudis tocantins, which is the sister-species to P. fusca (Garda \& Cannatella 2007); Proceratophrys cururu, the sisterspecies to $P$. renalis (Amaro et al. 2009); Phyllomedusa megacephala, the sister-species to $P$. rhodei (Faivovich et al. 2010); Phasmahyla jandaia, the sister-species to all other Atlantic endemic Phasmahyla species; Ischnocnema penaxavantinho, from I. verrucosa species series (Giaretta et al. 2007); Physalaemus deimaticus, belonging to the Atlantic $P$. deimaticus group (Nascimento et al. 2005); and Thoropa megatympanum, which belongs to an Atlantic genus. Cerrado endemics belonging to Amazonian and/or Andean clades include: Scinax constrictus, from the Amazonian S. rostratus group (Faivovich et al. 2005); Barycholus ternetzi, the sister-species to the transAndean B. pulcher (Hedges et al. 2008); and Oreobates heterodactylus, from a mostly Amazonian/Andean clade (Padial et al. 2008).

On the other hand, some Cerrado endemic species seem to be a product of in situ diversification. Some small clades are entirely composed of species endemic to the Cerrado, such as the three species in the clade of Ameerega flavopicta (Grant et al. 2006), the four species in Bokermannohyla pseudopseudis group (Faivovich et al. 2005), and the four species in the clade of Phylomedusa ayeaye (Faivovich et al. 2010). What is especially interesting about these clades is that they are partially sympatric, and species are restricted to areas with steeper relief and higher altitudes. This pattern 
reveals the importance of mountain ranges to Cerrado anuran in situ diversification. With the exception of $A$. flavopicta, all species show restricted ranges and are found on one mountaintop or mountain range. It is possible that future taxonomic and phylogenetic studies will reveal similar patterns in other clades, since many other species with restricted ranges were not included in the available phylogenies.

\section{Future directions}

This paper is a first approach to the description of the distribution patterns of Cerrado anurans. This system shows great potential for testing ecological and historical biogeographic hypotheses, due to its high richness and endemism and the occurrence of assemblages composed of representatives of different lineages; probably originating either in open domains or in adjoining tropical rain forests. Apart from the historical component, represented by multiple species pools and geographic barriers, we expect that climatic and elevation gradients, microhabitat availability, and species interactions may also be important factors determining species distribution and assemblage composition in the Cerrado. Testing these relationships may be a promising way of better understanding factors driving local and regional scale faunal assembly.

\section{References}

Amaro, R. C., D. Pavan \& M. T. Rodrigues (2009). On the generic identity of Odontophrynus moratoi Jim \& Caramaschi, 1980 (Anura, Cycloramphidae). Zootaxa 2071: 61-68.

Bastos, R. P. (2007). Anfíbios do Cerrado. Herpetologia no Brasil II. L. B. Nascimento and M. E. O. Oliveira. Belo Horizonte, Sociedade Brasileira de Herpetologia: 87-100.

Brasileiro, C. A., R. J. Sawaya, M. C. Kiefer \& M. Martins (2005). Amphibians of an open Cerrado fragment in southeastern Brazil. Biota Neotropica 5: 93-109.

Brasileiro, C. A., E. M. Lucas, H. M. Oyamaguchi, M. T. C. Thomé \& M. Dixo (2008). Anurans, northern Tocantins River Basin, states of Tocantins and Maranhão, Brazil. Check List 4: 185-197.

Buckley, L. B. \& W. Jetz (2007). Environmental and historical constraints on global patterns of amphibian richness. Proceedings of the Royal Society B: Biological Sciences 274: 1167-1173. 
Calió, M. F., J. R. Pirani \& L. Struwe (2008). Morphology-based phylogeny and revision of Prepusa and Senaea (Gentianaceae: Helieae) - rare endemics from eastern Brazil. Kew Bulletin 63: 169-191.

Caramaschi, U. (2010). Notes on the taxonomic status of Elachistocleis ovalis (Schneider, 1799) and description of five new species of Elachistocleis Parker, 1927 (Amphibia, Anura, Microhylidae). Boletim do Museu Nacional: 1-32.

Caramaschi, U., C. A. G. Cruz \& M. V. Segalla (2010). A new species of Hypsiboas of the $H$. polytaenius clade from the state of Paraná, Southern Brazil (Anura: Hylidae). South American Journal of Herpetology 5: 169-174.

Cardoso, M. W. \& J. P. P. Pombal-Jr (2010). A new species of small Scinax Wagler, 1830 (Amphibia, Anura, Hylidae) of the Scinax ruber clade from Cerrado of central Brazil. Amphibia-Reptilia 31: 411-418.

Carnaval, A. C. \& C. Moritz (2008). Historical climate modelling predicts patterns of current biodiversity in the Brazilian Atlantic forest. Journal of Biogeography 35: 1187-1201.

Colli, G. R., R. P. Bastos \& A. A. Araujo (2002). The Character and Dynamics of the Cerrado Herpetofauna. The Cerrados of Brazil: ecology and natural history of a neotropical savanna. P. S. Oliveira and R. J. Marquis. New York, Columbia University Press: 223-239.

Costa, L. P. (2003). The historical bridge between the Amazon and the Atlantic Forest of Brazil: a study of molecular phylogeography with small mammals. Journal of Biogeography 30: 71-86.

Crump, M. L. \& N. J. Scott Jr. (1994). Visual Encounter Surveys. Measuring and Monitoring Biodiversity: Standard Methods for Amphibians. W. R. Heyer, M. A. Donnelly, R. W. McDiarmid, L.-A. C. Hayek and M. S. Foster. Washington, Smithsonian Institution Press.

Diniz-Filho, J. A. F., L. M. Bini, C. M. Vieira, M. C. Souza, R. P. Bastos, D. Brandão \& L. G. Oliveira (2004). Spatial patterns in species richness and priority areas for conservation of anurans in the Cerrado region, Central Brazil. Amphibia-Reptilia 25: 63-75.

Diniz-Filho, J. A. F., L. M. Bini, M. P. Pinto, T. F. L. V. B. Rangel, P. Carvalho, S. L. Vieira \& R. P. Bastos (2007). Conservation biogeography of anurans in Brazilian Cerrado. Biodiversity and Conservation 16: 9971008.

Diniz-Filho, J. A. F., L. M. Bini, C. M. Vieira, D. Blamires, L. Terribile, R. Bastos, G. de Oliveira \& B. Barreto (2008). Spatial patterns of terrestrial vertebrate species richness in the Brazilian Cerrado. Zoological Studies 47: 146-157.

Diniz-Filho, J. A. F., R. P. Bastos, T. F. L. V. B. Rangel, L. M. Bini, P. Carvalho \& R. J. Silva (2005). Macroecological correlates and spatial patterns of anuran description dates in the Brazilian Cerrado. Global Ecology and Biogeography 14: 469-477.

Eterovick, P. C. \& I. Sazima (2000). Structure of an anuran community in a montane meadow in southeastern Brazil: effects of seasonality, habitat, and predation. Amphibia-Reptilia 21: 439-461.

Faivovich, J., C. F. B. Haddad, P. C. A. Garcia, D. R. Frost, J. A. Campbell \& W. C. Wheeler (2005). Systematic review of the frog family Hylidae, with special reference to Hylinae: phylogenetic analysis and taxonomic 
revision. Bulletin of the American Museum of Natural History 294: 1240.

Faivovich, J., C. F. B. Haddad, D. Baêta, K.-H. Jungfer, G. F. R. Álvares, R. A. Brandão, C. Sheil, L. S. Barrientos, C. L. Barrio-Amorós, C. A. G. Cruz \& W. C. Wheeler (2010). The phylogenetic relationships of the charismatic poster frogs, Phyllomedusinae (Anura, Hylidae). Cladistics 26: 227-261.

Frost, D. R., T. Grant, J. Faivovich, R. H. Bain, A. Haas, C. F. B. Haddad, R. O. de Sa, A. Channing, M. Wilkinson, S. C. Donnellan, C. J. Raxworthy, J. A. Campbell, B. L. Blotto, P. Moler, R. C. Drewes, R. A. Nussbaum, J. D. Lynch, D. M. Green \& W. C. Wheeler (2006). The amphibian tree of life. Bulletin of the American Museum of Natural History: 1-370.

Garda, A. A. \& D. C. Cannatella (2007). Phylogeny and biogeography of paradoxical frogs (Anura, Hylidae, Pseudae) inferred from $12 \mathrm{~S}$ and 16S mitochondrial DNA. Molecular Phylogenetics and Evolution. 44: 104-114.

Giaretta, A. A., D. Toffoli \& L. E. Oliveira (2007). A new species of Ischnocnema (Anura: Eleutherodactylinae) from open areas of the Cerrado Biome in southeastern Brazil. Zootaxa 1666: 43-51.

Giulietti, A. M. \& J. R. Pirani (1988). Patterns of geographic distribution of some plant species from the Espinhaço range, Minas Gerais and Bahia, Brazil. Workshop on Neotropical Distribution Patterns Rio De Janeiro, Academia Brasileira de Ciências.

Graham, C. H., S. Ferrier, F. Huettman, C. Moritz \& A. Peterson (2004). New developments in museum-based informatics and applications in biodiversity analysis. Trends in Ecology \& Evolution 19: 497-503.

Grant, T., D. R. Frost, J. P. Caldwell, R. Gagliardo, C. F. B. Haddad, P. J. R. Koko, D. B. Means, B. P. Noonan, W. E. Schargel \& W. C. Wheeler (2006). Phylogenetic systematics of dart-poison frogs and their relatives (Amphibia: Athesphatanura: Dendrobatidae). Bulletin of the American Museum of Natural History 299: 1-262.

Guo, Q. \& Y. Liu (2010). ModEco: an integrated software package for ecological niche modeling. Ecography 33: 1-6.

Hedges, S. B., W. E. Duellman \& M. P. Heinicke (2008). New World directdeveloping frogs (Anura : Terrarana): Molecular phylogeny, classification, biogeography, and conservation. Zootaxa: 1-182.

Heinicke, M. P., W. E. Duellman \& S. B. Hedges (2007). Major Caribbean and Central American frog faunas originated by ancient oceanic dispersal. Proceedings of the National Academy of Sciences of the United States of America. 104: 10092-10097.

Heyer, W. R. (1999). A new genus and species of frog from Bahia, Brazil (Amphibia: Anura: Leptodactylidae) with comments on the zoogeography of brazilian campos rupestres. Proceedings of the Biological Society of Washington 112: 19-39.

Hillman, S. S., P. C. Withers, R. C. Drewes \& S. D. Hillyard (2009). Ecological and Environmental Physiology of Amphibians. New York, Oxford University Press Inc.

Joly, C. A., M. P. M. Aidar, C. A. Klink, D. G. McGrath, A. G. Moreira, P. Moutinho, D. C. Nepstad, A. A. Oliveira, A. Pott, M. J. N. Rodal \& E. V. 
S. B. Sampaio (1999). Evolution of the Brazilian phytogeography classification systems: implications for biodiversity conservation. Ciencia e Cultura 51: 331-348.

Laffan, S. W., E. Lubarsky \& D. F. Rosauer (2010). Biodiverse, a tool for the spatial analysis of biological and related diversity. Ecography: 643-647.

Leite, F. S. F., F. A. Juncá \& P. C. Eterovick (2008). Status do conhecimento, endemismo e conservação de anfíbios anuros da Cadeia do Espinhaço, Brasil. Megadiversidade 4: 182-200.

Lester, S. E., B. I. Ruttenberg, S. D. Gaines \& B. P. Kinlan (2007). The relationship between dispersal ability and geographic range size. Ecology Letters 10: 745-758.

McDonald, R., M. McKnight, D. Weiss, E. Selig, M. O'Connor, C. Violin \& A. Moody (2005). Species compositional similarity and ecoregions: Do ecoregion boundaries represent zones of high species turnover? Biological Conservation 126: 24-40.

Nascimento, L. B., U. Caramaschi \& C. A. G. Cruz (2005). Taxonomic review of the species groups of the genus Physalaemus Fitzinger, 1826 with revalidation of the genera Engystomops Jiménez-de-la-Espada, 1872 and Eupemphix Steindachner, 1863 (Amphibia, Anura, Leptodactylidae). Arquivos do Museu Nacional 63: 297-320.

Nunes, I., R. R. Carvalho-Jr. \& E. G. Pereira (2010). A new species of Scinax Wagler (Anura: Hylidae) from Cerrado of Brazil. Zootaxa 2514: 24-34.

Olson, D. M., E. Dinerstein, E. D. Wikramanayake, N. D. Burgess, G. V. N. Powell, E. C. Underwood, J. A. D'Amico, I. Itoua, H. E. Strand, J. C. Morrison, C. J. Loucks, T. F. Allnut, T. H. Ricketts, Y. Kura, J. F. Lamoreux, W. W. Wettengel, P. Hedao \& K. R. Kassem (2001). Terrestrial ecoregions of the world: a new map of life on earth. BioScience 51: 933-938.

Padial, J. M., J. C. Chaparro \& I. de-la-Riva (2008). Systematics of Oreobates and the Eleutherodactylus discoidalis species group (Amphibia, Anura), based on two mitochondrial DNA genes and external morphology. Zoological Journal of the Linnean Society 152: 737-773.

Pugliese, A., D. Baêta \& J. P. Pombal-Jr (2009). A new species of Scinax (Anura: Hylidae) from rocky montane fields in southeastern and central Brazil. Zootaxa 2269: 53-64.

Rahbek, C., N. J. Gotelli, R. K. Colwell, G. L. Entsminger, T. F. L. V. B. Rangel \& G. R. Graves (2007). Predicting continental-scale patterns of bird species richness with spatially explicit models. Proceedings of the Royal Society B: Biological Sciences 274: 165-174.

Rangel, T. F. L. V. B., J. A. F. Diniz-Filho \& L. M. Bini (2010). SAM: a comprehensive application for spatial analysis in macroecology. Ecography 33: 46-50.

Safford, H. F. (1999). Brazilian Paramos I. An introduction to the physical environment and vegetation of the campos de altitude. Journal of Biogeography 26: 693-712.

Silva, J. F., M. R. Fariñas, J. M. Felfili \& C. A. Klink (2006). Spatial heterogeneity, land use and conservation in the cerrado region of Brazil. Journal of Biogeography 33: 536-548. 
Silva, J. M. C. (1996). Distribution of Amazonian and Atlantic Forest Birds in Gallery Forests of the Cerrado Region, South America. Ornitologia Neotropical 7: 1-18.

Silva, J. M. C. \& J. M. Bates (2002). Biogeographic patterns and conservation in the South American Cerrado: a tropical savanna hotspot. Bioscience 52: 225-234.

Silvano, D. L. \& M. V. Segalla (2005). Conservation of Brazilian amphibians. Conservation Biology 19: 653-658.

Toledo, L. F. (2010). Description of a new species of Pseudopaludicola Miranda-Ribeiro, 1926 from the state of São Paulo, Southeastern Brazil (Anura, Leiuperidae). Zootaxa 2681: 47-56.

Valdujo, P. H., R. S. Recoder, M. M. Vasconcellos \& A. S. Portella (2009). Amphibia, Anura, São Desidério, western Bahia uplands, northeastern Brazil. Check List 5: 903-911.

Vaz-Silva, W., A. G. Guedes, P. L. Azevedo-Silva, F. F. Gontijo, R. S. Barbosa, G. R. Aloísio \& F. C. G. Oliveira (2007). Herpetofauna, Espora hydroelectric power plant, state of Goiás, Brazil. Check List 3: 338-345. 
Appendix. Anuran species distributed in Cerrado, degree of association to the domain (typical or marginal), voucher specimen or citation, and collection locality. Abreviations: Assoc - degree of association with the Cerrado, Distrib distribution pattern, E - Cerrado endemic, NE - near endemic, O - open domains, W - widespread, T - typical, AT - species that occur both in Atlantic Forest and Cerrado, AM - species that occur both in Amazonia and Cerrado, $\mathrm{CA}$ - species that occur both in Caatinga and Cerrado, $\mathrm{CH}$ - species that occur in chaco and / or panatanal, and Cerrado, S - species with meridional distribution occurring in Cerrado. Asterisks indicate species not referred by previous assessments. Species marked with "n/d" are still to be deposited in a zoological collection.

\begin{tabular}{|c|c|c|c|c|}
\hline Espécie & Assoc & Distrib & Voucher / citation & Locality \\
\hline \multicolumn{5}{|l|}{ Aromobatidae } \\
\hline & & & & Chapada dos \\
\hline Allobates brunneus* & $\mathrm{T}$ & $E$ & ANSP 11241 & Guimarães, MT \\
\hline Allobates goianus & $\mathrm{T}$ & $E$ & MZUSP 73706 & Alto Paraíso, GO \\
\hline Allobates aff. brunneus* & $\mathrm{T}$ & $E$ & MZUSP 127129 & Guaraí, TO \\
\hline \multicolumn{5}{|l|}{ Brachycephalidae } \\
\hline $\begin{array}{l}\text { Ischnocnema } \\
\text { penaxavantinho* }\end{array}$ & $\mathrm{T}$ & $E$ & ZUEC 13639 & Uberlândia, MG \\
\hline Ischnocnema aff. & & & & \\
\hline paulodutrai* & $\mathrm{T}$ & $E$ & $\mathrm{n} / \mathrm{d}$ & PN Peruaçu \\
\hline \multicolumn{5}{|l|}{ Bufonidae } \\
\hline \multicolumn{5}{|l|}{ Melanophryniscus } \\
\hline fulvoguttatus* & $\mathrm{T}$ & $\mathrm{CHA}$ & CHUNB 43325 & Bodoquena, MS \\
\hline Rhaebo guttatus & $\mathrm{T}$ & AM & CHUNB 43645 & Alto Paraíso, GO \\
\hline Rhinella azarai* & $\mathrm{M}$ & $\mathrm{CHA}$ & El 1438 & Bela Vista, MS \\
\hline Rhinella cerradensis* & $\mathrm{T}$ & $\mathrm{E}$ & CHUNB 13790 & Mineiros, GO \\
\hline Rhinella granulosa & $\mathrm{M}$ & W & MZUSP 105782 & Pirapora, MG \\
\hline Rhinella icterica* & $\mathrm{M}$ & AT & MZUSP 28389 & Botucatu, SP \\
\hline Rhinella major* & $\mathrm{M}$ & AM & MZUSP 128246 & $\begin{array}{l}\text { Cuiabá, MT } \\
\text { Chapada dos }\end{array}$ \\
\hline Rhinella margaritifer & $\mathrm{T}$ & W & CHUNB 15589 & Guimarães, MT \\
\hline Rhinella mirandaribeiroi* & $\mathrm{T}$ & NE & MZUSP 71308 & Serra da Mesa, GO \\
\hline Rhinella ocellata & $\mathrm{T}$ & NE & CHUNB 28128 & Alcinópolis, MS \\
\hline Rhinella ornata & $\mathrm{M}$ & AT & MZUSP 13848 & Botucatu, SP \\
\hline Rhinella pombali & $\mathrm{M}$ & AT & MNRJ 22234 & Grão Mogol, MG \\
\hline Rhinella rubescens & $\mathrm{T}$ & $\mathrm{NE}$ & CHUNB 32249 & Brasília, DF \\
\hline Rhinella schneideri & $\mathrm{T}$ & W & CHUNB 14237 & Brasília, DF \\
\hline Rhinella scitula* & $\mathrm{T}$ & $E$ & MNRJ 25894 & Bonito, MS \\
\hline Rhinella aff. pombali* & $\mathrm{T}$ & $E$ & CHUNB 51110 & São Desidério, BA \\
\hline Rhinella veredas* & $\mathrm{T}$ & $E$ & CHUNB 38652 & Cocos, BA \\
\hline \multicolumn{5}{|l|}{ Centrolenidae } \\
\hline Vitreorana aff. eurygnata & $\mathrm{M}$ & AT & ZUEC 2242 & Santana do Riacho, MG \\
\hline Vitreorana uranoscopa* & $\mathrm{M}$ & AT & ZUEC 3679 & Botucatu, SP \\
\hline \multicolumn{5}{|l|}{ Ceratophrydae } \\
\hline Ceratophrys aurita* & M & AT & CHUNB 38786 & Buritizeiro, MG \\
\hline
\end{tabular}




\begin{tabular}{|c|c|c|c|c|}
\hline Espécie & Assoc & Distrib & Voucher / citation & Locality \\
\hline \multicolumn{5}{|l|}{ Odontophrynus } \\
\hline americanus & M & S & CHUNB 49294 & Bonito, MS \\
\hline Odontophrynus cultripes & $\mathrm{T}$ & NE & NHMW 16522 & Lagoa Santa, MG \\
\hline Odontophrynus salvatori & $\mathrm{T}$ & $E$ & MNRJ 15870 & Alto Paraíso, GO \\
\hline Proceratophrys boiei* & M & AT & Leite et al (2009) & Cristália, MG \\
\hline Proceratophrys & & & Amaro et al & \\
\hline concavitympanum & $\mathrm{T}$ & AM & $(2009)$ & Palmas, TO \\
\hline Proceratophrys cururu & $\mathrm{T}$ & $E$ & ZUEC 9557 & Serra do Cipó, MG \\
\hline Proceratophrys goyana & $\mathrm{T}$ & $E$ & MNRJ 296 & Serra da Mesa, GO \\
\hline $\begin{array}{l}\text { Proceratophrys moratoi } \\
\text { Thoropa }\end{array}$ & $\mathrm{T}$ & $E$ & CFBH 12854 & Itirapina, SP \\
\hline megatympanum & $\mathrm{T}$ & NE & MZUSP 56761 & Jaboticatubas, MG \\
\hline Thoropa miliaris* & M & AT & Leite et al (2009) & Botumirim, MG \\
\hline \multicolumn{5}{|l|}{ Dendrobatidae } \\
\hline Adelphobates & & & & \\
\hline galactonotus* & M & AM & MZUSP 133222 & $\begin{array}{l}\text { Palmas, TO } \\
\text { Chapada dos }\end{array}$ \\
\hline Ameerega braccata & $\mathrm{T}$ & $E$ & UFMT 7700 & Guimarães, MT \\
\hline Ameerega flavopicta & $\mathrm{T}$ & $E$ & CHUNB 14925 & Brasília, DF \\
\hline Ameerega picta & $\mathrm{T}$ & NE & MNRJ 25471 & Bonito, MS \\
\hline Ameerega aff. picta* & $\mathrm{T}$ & NE & $\mathrm{n} / \mathrm{d}$ & Barra do Garças, MT \\
\hline \multicolumn{5}{|l|}{ Hylidae } \\
\hline Aplastodiscus & & & & \\
\hline leucopygius* & M & AT & MZUSP 74436 & Botucatu, SP \\
\hline $\begin{array}{l}\text { Aplastodiscus perviridis } \\
\text { Bokermannohyla }\end{array}$ & $\mathrm{T}$ & AT & CHUNB 17012 & Brasília, DF \\
\hline $\begin{array}{l}\text { alvarengai } \\
\text { Bokermannohyla }\end{array}$ & $\mathrm{T}$ & NE & MZUSP 58264 & Grão Mogol, MG \\
\hline ibitiguara & $\mathrm{T}$ & $E$ & MZUSP 72288 & Alpinópolis, MG \\
\hline Bokermannohyla & & & & \\
\hline $\begin{array}{l}\text { izecksohni* } \\
\text { Bokermannohyla }\end{array}$ & M & AT & MZUSP 50178 & $\begin{array}{l}\text { Botucatu, SP } \\
\text { Serra do Cipó (usina), }\end{array}$ \\
\hline $\begin{array}{l}\text { nanuzae } \\
\text { Bokermannohyla }\end{array}$ & $\mathrm{T}$ & NE & MZUSP 73648 & MG \\
\hline pseudopseudis & $\mathrm{T}$ & $E$ & CHUNB 43650 & Alto Paraíso, GO \\
\hline $\begin{array}{l}\text { Bokermannohyla ravida } \\
\text { Bokermannohyla }\end{array}$ & $\mathrm{T}$ & $E$ & MZUSP 68498 & Presidente Olegário, MG \\
\hline $\begin{array}{l}\text { saxicola } \\
\text { Bokermannohyla }\end{array}$ & $\mathrm{T}$ & $E$ & MZUSP 76925 & $\begin{array}{l}\text { Serra do Cipó, MG } \\
\text { São Roque de Minas, }\end{array}$ \\
\hline sazimai & $\mathrm{T}$ & $E$ & MNRJ 4149 & MG \\
\hline $\begin{array}{l}\text { Corythomantis greeningi } \\
\text { Dendropsophus }\end{array}$ & $\mathrm{M}$ & CA & CFBH 10211 & Grão Mogol, MG \\
\hline anataliasiasi & $\mathrm{T}$ & $E$ & MZUSP 73790 & Brejinho de Nazaré, TO \\
\hline $\begin{array}{l}\text { Dendropsophus anceps* } \\
\text { Dendropsophus }\end{array}$ & M & AT & CCJJ 7819 & Borebi, SP \\
\hline araguaya & $\mathrm{T}$ & $E$ & MZUSP 66796 & Alto Araguaia, MT \\
\hline $\begin{array}{l}\text { Dendropsophus branneri } \\
\text { Dendropsophus }\end{array}$ & M & AT & MCN 2831 & Avaré, SP \\
\hline cerradensis & $\mathrm{T}$ & $E$ & MNRJ 17293 & Ribas do Rio Pardo, MS \\
\hline $\begin{array}{l}\text { Dendropsophus cruzi } \\
\text { Dendropsophus }\end{array}$ & $\mathrm{T}$ & $E$ & CFBH 2939 & Silvânia, GO \\
\hline elianeae & $\mathrm{T}$ & NE & MNRJ 17297 & Bela Vista, MS \\
\hline $\begin{array}{l}\text { Dendropsophus jimi } \\
\text { Dendropsophus }\end{array}$ & $\mathrm{T}$ & NE & MNRJ 21980 & Botucatu, SP \\
\hline $\begin{array}{l}\text { leucophyllatus* } \\
\text { Dendropsophus }\end{array}$ & M & AM & CHUNB 14250 & $\begin{array}{l}\text { Palmas, TO } \\
\text { Chapada dos }\end{array}$ \\
\hline melanargyreus & $\mathrm{T}$ & W & UFMT 1409 & Guimarães, MT \\
\hline Dendropsophus & M & AT & JJ 7853 & Avaré, SP \\
\hline
\end{tabular}




\begin{tabular}{|c|c|c|c|c|}
\hline Espécie & Assoc & Distrib & Voucher / citation & Locality \\
\hline \multicolumn{5}{|l|}{ microps* } \\
\hline Dendropsophus minutus & $\mathrm{T}$ & W & CHUNB 42497 & Brasília, DF \\
\hline Dendropsophus nanus & $\mathrm{T}$ & W & ZUFG 2521 & Serranópolis, GO \\
\hline $\begin{array}{l}\text { Dendropsophus rhea } \\
\text { Dendropsophus }\end{array}$ & $\mathrm{T}$ & $E$ & MZUSP 9104 & Pirassununga, SP \\
\hline $\begin{array}{l}\text { rubicundulus } \\
\text { Dendropsophus }\end{array}$ & $\mathrm{T}$ & NE & CHUNB 52345 & Lagoa Santa, MG \\
\hline sanborni* & M & $\mathrm{S}$ & ZUEC 12040 & Itirapina, SP \\
\hline $\begin{array}{l}\text { Dendropsophus soaresi } \\
\text { Dendropsophus }\end{array}$ & $\mathrm{M}$ & $\mathrm{CA}$ & CHUNB 34060 & $\begin{array}{l}\text { Chapada Gaúcha, MG } \\
\text { Cuiabá (São Vicente - }\end{array}$ \\
\hline $\begin{array}{l}\text { tritaeniatus } \\
\text { Hypsiboas }\end{array}$ & $\mathrm{T}$ & $E$ & MZUSP 73656 & Gustavo Dutra), MT \\
\hline $\begin{array}{l}\text { albomarginatus* } \\
\text { Hypsiboas }\end{array}$ & $\mathrm{M}$ & AT & MZUSP 3068 & Pirassununga, SP \\
\hline albopunctatus & $\mathrm{T}$ & W & ZUFG 3290 & Mineiros, GO \\
\hline Hypsiboas boans* & $\mathrm{M}$ & AM & CHUNB 11143 & Palmas, TO \\
\hline Hypsiboas botumirim* & $\mathrm{T}$ & $E$ & MNRJ 40886 & Botumirim, MG \\
\hline Hypsiboas buriti & $\mathrm{T}$ & $\mathrm{E}$ & MNRJ 23476 & Buriti, MG \\
\hline Hypsiboas caingua* & M & AT & MZUSP 16149 & Botucatu, SP \\
\hline Hypsiboas cipoensis & $\mathrm{T}$ & $E$ & MNRJ 4039 & Jaboticatubas, MG \\
\hline Hypsiboas crepitans & $\mathrm{T}$ & W & CHUNB 44561 & Buritizeiro, MG \\
\hline Hypsiboas ericae & $\mathrm{T}$ & $E$ & MNRJ 15875 & Alto Paraíso, GO \\
\hline $\begin{array}{l}\text { Hypsiboas faber } \\
\text { Hypsiboas }\end{array}$ & M & AT & ZUEC 12024 & Itirapina, SP \\
\hline geographicus* & $\mathrm{T}$ & W & CHUNB 25729 & Alcinópolis, MS \\
\hline Hypsiboas goianus & $\mathrm{T}$ & $E$ & MNRJ 3235 & São João d'Aliança, GO \\
\hline Hypsiboas lundii & $\mathrm{T}$ & NE & ZUFG 3495 & Goiânia, GO \\
\hline Hypsiboas multifasciatus & $\mathrm{T}$ & AM & MNRJ 44713 & Balsas, MA \\
\hline Hypsiboas phaeopleura & $\mathrm{T}$ & $E$ & MNRJ 19893 & Alto Paraíso, GO \\
\hline Hypsiboas polytaenius & $M$ & AT & MZUSP 7832 & Botucatu, SP \\
\hline Hypsiboas prasinus & $M$ & AT & CFBH 2122 & Jaguaraiaíva, PR \\
\hline Hypsiboas punctatus & $\mathrm{T}$ & W & CHUNB 25727 & Alcinópolis, MS \\
\hline $\begin{array}{l}\text { Hypsiboas raniceps } \\
\text { Hypsiboas aff. }\end{array}$ & $\mathrm{T}$ & W & CHUNB 43635 & Brasília, DF \\
\hline $\begin{array}{l}\text { leucocheila* } \\
\text { Hypsiboas }\end{array}$ & $\mathrm{T}$ & $E$ & CHUNB 24006 & Britânia, GO \\
\hline $\begin{array}{l}\text { jaguariaivensis* } \\
\text { Hypsiboas }\end{array}$ & $\mathrm{T}$ & $E$ & MNRJ 66203 & Jaguariaiva, PR \\
\hline stenocephalus & $\mathrm{T}$ & NE & MNRJ 23474 & Sacramento, MG \\
\hline Itapotihyla langsdorffii & $M$ & AT & ZUEC 12025 & Itirapina, SP \\
\hline Lysapsus caraya & $\mathrm{T}$ & $E$ & CHUNB 42737 & Britânia, GO \\
\hline Osteocephalus taurinus & $\mathrm{T}$ & AM & CHUNB51740 & $\begin{array}{l}\text { Carolina, MA } \\
\text { Serra do Cipó, Km 126, }\end{array}$ \\
\hline Phasmahyla jandaia & $\mathrm{T}$ & NE & MZUSP 74443 & MG \\
\hline Phyllomedusa araguari & $\mathrm{T}$ & $E$ & ZUEC 12880 & Perdizes, MG \\
\hline Phyllomedusa ayeaye & $\mathrm{T}$ & NE & CFBH 15672 & Pedregulho, SP \\
\hline Phyllomedusa azurea & $\mathrm{T}$ & NE & MNRJ 27839 & Mambaí, GO \\
\hline $\begin{array}{l}\text { Phyllomedusa bahiana } \\
\text { Phyllomedusa }\end{array}$ & M & AT & CFBH 10205 & Grão Mogol, MG \\
\hline burmeisteri & M & AT & MNRJ 10094 & $\begin{array}{l}\text { Botucatu, SP } \\
\text { Chapada dos }\end{array}$ \\
\hline $\begin{array}{l}\text { Phyllomedusa centralis } \\
\text { Phyllomedusa }\end{array}$ & $\mathrm{T}$ & $\mathrm{E}$ & CFBH 15794 & Guimarães, MT \\
\hline $\begin{array}{l}\text { hypochondrialis } \\
\text { Phyllomedusa }\end{array}$ & M & AM & MNRJ 43920 & Goiatins, TO \\
\hline $\begin{array}{l}\text { megacephala } \\
\text { Phyllomedusa }\end{array}$ & $\mathrm{T}$ & $E$ & MZUSP 134313 & Serra do Cipó, MG \\
\hline nordestina* & $\mathrm{T}$ & $\mathrm{CA}$ & CHUNB 38438 & Flores de Goiás, GO \\
\hline
\end{tabular}




\begin{tabular}{|c|c|c|c|c|}
\hline Espécie & Assoc & Distrib & Voucher / citation & Locality \\
\hline Phyllomedusa oreades & $\mathrm{T}$ & $E$ & CFBH 15796 & Pirenópolis, GO \\
\hline Phyllomedusa sauvagii* & $\mathrm{T}$ & $\mathrm{CHA}$ & CFBH 14250 & Bonito, MS \\
\hline Phyllomedusa distincta & $M$ & AT & CFBH 21022 & Jaguaraiaíva, PR \\
\hline Pseudis bolbodactyla & $\mathrm{T}$ & AT & CHUNB 38380 & Flores de Goiás, GO \\
\hline Pseudis platensis & M & $\mathrm{CHA}$ & MNRJ 41616 & Corumbá, MS \\
\hline Pseudis tocantins & $\mathrm{T}$ & $E$ & MNRJ 2481 & Porto Nacional, TO \\
\hline Scinax acuminatus & $M$ & $\mathrm{CHA}$ & CFBH 14195 & Poconé, MT \\
\hline Scinax cabralensis* & $\mathrm{T}$ & $E$ & MNRJ 42883 & $\begin{array}{l}\text { Joaquim Felício, MG } \\
\text { PN Serra da Canastra, } \\
\text { São Roque de Minas, }\end{array}$ \\
\hline Scinax canastrensis & $\mathrm{T}$ & $E$ & MNRJ 4147 & MG \\
\hline Scinax centralis & $\mathrm{T}$ & $E$ & MNRJ 17465 & Silvânia, GO \\
\hline Scinax constrictus & $\mathrm{T}$ & $E$ & MNRJ 31205 & $\begin{array}{l}\text { Palmeiras, GO } \\
\text { Serra do Cipó, Alto }\end{array}$ \\
\hline Scinax curicica & $\mathrm{T}$ & $\mathrm{NE}$ & MNRJ 26327 & Palácio, MG \\
\hline Scinax fuscomarginatus & $\mathrm{T}$ & W & MNRJ 33061 & Buritizeiro, MG \\
\hline Scinax fuscovarius & $\mathrm{T}$ & W & MNRJ 19942 & Ivolândia, GO \\
\hline Scinax hiemalis* & $M$ & AT & CFBH 4188 & Botucatu, SP \\
\hline Scinax longilineus* & $M$ & AT & Leite et al (2009) & Brumadinho, MG \\
\hline Scinax lutzorum* & $\mathrm{T}$ & $E$ & MNRJ 51438 & Aragominas, TO \\
\hline Scinax machadoi & $\mathrm{T}$ & NE & CFBH 6245 & Jaboticatubas, MG \\
\hline Scinax maracaya & $\mathrm{T}$ & $E$ & CFBH 16 & Alpinópolis, MG \\
\hline Scinax nasicus* & $\mathrm{T}$ & $\mathrm{O}$ & CFBH 14246 & Bonito, MS \\
\hline Scinax pinima & $\mathrm{T}$ & $E$ & MNRJ 4999 & Jaboticatubas, MG \\
\hline Scinax rogerioi* & $\mathrm{T}$ & $E$ & CHUNB 49959 & Brasília, DF \\
\hline Scinax similis* & $M$ & AT & Silva et al 2008 & Icém, SP \\
\hline $\begin{array}{l}\text { Scinax sp gr catharinae* } \\
\text { Scinax sp2 gr }\end{array}$ & $\mathrm{T}$ & $E$ & CHUNB 40894 & Luziânia, DF \\
\hline $\begin{array}{l}\text { catharinae* } \\
\text { Scinax aff }\end{array}$ & $\mathrm{T}$ & $E$ & $\mathrm{n} / \mathrm{d}$ & \\
\hline cruentommus* & $\mathrm{T}$ & $E$ & $\mathrm{n} / \mathrm{d}$ & Barra do Garças, MT \\
\hline Scinax squalirostris & $\mathrm{T}$ & S & ZUFG 2717 & Cristalina, GO \\
\hline Scinax tigrinus* & $\mathrm{T}$ & $E$ & CHUNB 13165 & Brasília, DF \\
\hline $\begin{array}{l}\text { Scinax } x \text {-signatus } \\
\text { Sphaenorhynchus }\end{array}$ & $\mathrm{T}$ & W & MNRJ 34620 & Pirapora, MG \\
\hline caramaschii* & $M$ & AT & CCJJ 7815 & Avaré, SP \\
\hline $\begin{array}{l}\text { Trachycephalus atlas* } \\
\text { Trachycephalus }\end{array}$ & M & $\mathrm{CA}$ & MNRJ 1178 & Barreiras, BA \\
\hline $\begin{array}{l}\text { mambaiensis* } \\
\text { Trachycephalus }\end{array}$ & $\mathrm{T}$ & $E$ & MZUSP 135715 & Mambai, BA \\
\hline $\begin{array}{l}\text { nigromaculatus } \\
\text { Trachycephalus }\end{array}$ & M & AT & MNRJ 38859 & João Pinheiro, MG \\
\hline venulosus & $\mathrm{T}$ & W & ZUFG 736 & Pontalina, GO \\
\hline \multicolumn{5}{|l|}{ Hylodidae } \\
\hline $\begin{array}{l}\text { Crossodactylus } \\
\text { bokermanni } \\
\text { Crossodactylus }\end{array}$ & $\mathrm{T}$ & $E$ & JJ 6044 & Serra do Cipó, MG \\
\hline cyclospinus & $M$ & AT & MNRJ 40220 & Cristália, MG \\
\hline $\begin{array}{l}\text { Crossodactylus sp. * } \\
\text { Crossodactylus }\end{array}$ & $\mathrm{T}$ & $E$ & MNRJ 50796 & Passos, MG \\
\hline trachystomus & $\mathrm{T}$ & NE & MNRJ 38466 & $\begin{array}{l}\text { Santana do Riacho, MG } \\
\text { Jaboticatubas, Serra do }\end{array}$ \\
\hline Hylodes otavioi & $\mathrm{T}$ & $E$ & MNRJ 4163 & Cipó, MG \\
\hline \multicolumn{5}{|l|}{ Leiuperidae } \\
\hline Eupemphix nattereri & $\mathrm{T}$ & NE & MZUSP 95173 & Cuiabá, MT \\
\hline $\begin{array}{l}\text { Physalaemus albifrons* } \\
\text { Physalaemus }\end{array}$ & M & $\mathrm{CA}$ & MNRJ 1109 & Barreiras, BA \\
\hline albonotatus & M & $\mathrm{CHA}$ & MNRJ 45099 & Cuiabá, MT \\
\hline
\end{tabular}




\begin{tabular}{|c|c|c|c|c|}
\hline Espécie & Assoc & Distrib & Voucher / citation & Locality \\
\hline \multicolumn{5}{|l|}{ Physalaemus } \\
\hline biligonigerus* & $\mathrm{M}$ & $\mathrm{s}$ & & \\
\hline Physalaemus centralis & $\mathrm{T}$ & $\mathrm{NE}$ & CHUNB 16245 & Brasília, DF \\
\hline Physalaemus cicada* & $\mathrm{M}$ & CA & MZUSP & Peruaçu, MG \\
\hline $\begin{array}{l}\text { Physalaemus cuvieri } \\
\text { Physalaemus }\end{array}$ & $\mathrm{T}$ & W & CHUNB 44765 & Brasília, DF \\
\hline $\begin{array}{l}\text { deimaticus } \\
\text { Physalaemus }\end{array}$ & $\mathrm{T}$ & $\mathrm{E}$ & MZUSP 56850 & Serra do Cipó, MG \\
\hline evangelistai & $\mathrm{T}$ & $\mathrm{NE}$ & MZUSP 76570 & Serra do Cipó, MG \\
\hline $\begin{array}{l}\text { Physalaemus gracilis* } \\
\text { Physalaemus }\end{array}$ & M & $S$ & $n / d$ & Jaguaraiaíva, PR \\
\hline marmoratus & $\mathrm{T}$ & $\mathrm{NE}$ & $\begin{array}{l}\text { CHUNB } 34027 \\
\text { Teixeira et al }\end{array}$ & Chapada Gaúcha, MG \\
\hline Physalaemus olfersii ${ }^{*}$ & $\mathrm{M}$ & AT & 2008 & Botucatu, SP \\
\hline Physalaemus sp.* & $\mathrm{T}$ & $\mathrm{E}$ & $n / d$ & Barra do Garças, MT \\
\hline $\begin{array}{l}\text { Pleurodema diplolister* } \\
\text { Pseudopaludicola }\end{array}$ & $\mathrm{T}$ & $\mathrm{CA}$ & CHUNB 22061 & $\begin{array}{l}\text { Cocos, BA } \\
\text { Vila Bela de Santíssima }\end{array}$ \\
\hline $\begin{array}{l}\text { boliviana } \\
\text { Pseudopaludicola }\end{array}$ & $\mathrm{M}$ & $\mathrm{CHA}$ & MZUSP 52102 & Trindade, MT \\
\hline $\begin{array}{l}\text { falcipes } \\
\text { Pseudopaludicola }\end{array}$ & $\mathrm{M}$ & $S$ & MZUSP 27081 & Planura, MG \\
\hline $\begin{array}{l}\text { mineira } \\
\text { Pseudopaludicola }\end{array}$ & $\mathrm{T}$ & $\mathrm{E}$ & MZUSP 55449 & $\begin{array}{l}\text { Serra do Cipó, MG } \\
\text { Aldeia Velha, Serra do }\end{array}$ \\
\hline mystacalis & $\mathrm{T}$ & W & MZUSP 4400 & Roncador, MT \\
\hline $\begin{array}{l}\text { Pseudopaludicola saltica } \\
\text { Pseudopaludicola }\end{array}$ & $\mathrm{T}$ & $E$ & MZUSP 25350 & Brasília, DF \\
\hline $\begin{array}{l}\text { serrana* } \\
\text { Pseudopaludicola }\end{array}$ & $\mathrm{T}$ & $\mathrm{NE}$ & ZUEC 2323 & Santana do Riacho, MG \\
\hline ternetzi & $\mathrm{T}$ & $E$ & MZUSP 27022 & Jataí, GO \\
\hline \multicolumn{5}{|l|}{ Leptodactylidae } \\
\hline $\begin{array}{l}\text { Leptodactylus } \\
\text { bokermanni } \\
\text { Leptodactylus }\end{array}$ & $\mathrm{M}$ & AT & MZUSP 6388 & Botucatu, SP \\
\hline $\begin{array}{l}\text { camaquara } \\
\text { Leptodactylus }\end{array}$ & $\mathrm{T}$ & $\mathrm{E}$ & MZUSP 74291 & $\begin{array}{l}\text { Serra do Cipó, MG } \\
\text { Manso, Chapada dos }\end{array}$ \\
\hline $\begin{array}{l}\text { chaquensis } \\
\text { Leptodactylus }\end{array}$ & $\mathrm{T}$ & $\mathrm{CHA}$ & UFMT 1734 & Guimarães, MT \\
\hline cunicularius & $\mathrm{T}$ & NE & CFBH 782 & Santana do Riacho, MG \\
\hline Leptodactylus elenae* & $\mathrm{M}$ & $\mathrm{CHA}$ & UFMS 725 & Bonito, MS \\
\hline Leptodactylus furnarius & $\mathrm{T}$ & NE & CHUNB 13743 & Mineiros, GO \\
\hline $\begin{array}{l}\text { Leptodactylus fuscus } \\
\text { Leptodactylus }\end{array}$ & $\mathrm{T}$ & W & CHUNB 44244 & Buritizeiro, MG \\
\hline $\begin{array}{l}\text { hylaedactylus } \\
\text { Leptodactylus }\end{array}$ & $\mathrm{T}$ & AM & CFBH 11405 & Babaçulândia \\
\hline labyrinthicus & $\mathrm{T}$ & W & CHUNB 38761 & São João d'Aliança, GO \\
\hline Leptodactylus latrans & $\mathrm{T}$ & W & MZUSP 134142 & $\begin{array}{l}\text { Botucatu, SP } \\
\text { Vila Bela de Santíssima }\end{array}$ \\
\hline $\begin{array}{l}\text { Leptodactylus lineatus* } \\
\text { Leptodactylus }\end{array}$ & $\mathrm{M}$ & AM & MZUSP 91746 & Trindade, MT \\
\hline macrosternum* & $\mathrm{T}$ & $\mathrm{CA}$ & & \\
\hline $\begin{array}{l}\text { Leptodactylus martinezi } \\
\text { Leptodactylus }\end{array}$ & $\mathrm{T}$ & $\mathrm{E}$ & ZUFG 3124 & Mineiros, GO \\
\hline $\begin{array}{l}\text { mystaceus } \\
\text { Leptodactylus }\end{array}$ & $\mathrm{T}$ & W & MNRJ 38820 & João Pinheiro, MG \\
\hline mystacinus & $\mathrm{T}$ & AT & CHUNB 25036 & Brasília, DF \\
\hline $\begin{array}{l}\text { Leptodactylus petersii } \\
\text { Leptodactylus }\end{array}$ & M & AM & MZUSP 128554 & Petrolina de Goiás, GO \\
\hline plaumanni* & $M$ & $\mathrm{~S}$ & CFBH 21038 & Jaguaraiaíva, PR \\
\hline
\end{tabular}




\section{Capítulo2}

\section{A historical perspective on the distribution of anuran}

amphibians in Brazilian Cerrado

Valdujo, P. H. \& M. Martins. Manuscrito em preparação que será submetido ao periódico Journal of Biogeography 


\begin{tabular}{|c|c|c|c|c|}
\hline Espécie & Assoc & Distrib & Voucher / citation & Locality \\
\hline $\begin{array}{l}\text { Leptodactylus } \\
\text { podicipinus }\end{array}$ & $\mathrm{T}$ & $\mathrm{O}$ & MNRJ 1039 & $\begin{array}{l}\text { Barreiras, BA (at or } \\
\text { near) }\end{array}$ \\
\hline Leptodactylus pustulatus & $\mathrm{T}$ & NE & CHUNB 14922 & Barra do Garças, MT \\
\hline Leptodactylus sertanejo* & $\mathrm{T}$ & $\mathrm{E}$ & ZUEC 13657 & $\begin{array}{l}\text { Uberlândia, MG } \\
\text { UHE Manso, Chapada }\end{array}$ \\
\hline Leptodactylus syphax & $\mathrm{T}$ & $\mathrm{O}$ & MZUSP 101015 & dos Guimarães, MT \\
\hline $\begin{array}{l}\text { Leptodactylus tapiti } \\
\text { Leptodactylus }\end{array}$ & $\mathrm{T}$ & $E$ & CHUNB 49534 & Alto Paraíso, GO \\
\hline troglodytes & $\mathrm{T}$ & $\mathrm{CA}$ & CHUNB 41976 & Mateiros, TO \\
\hline Leptodactylus vastus* & $\mathrm{M}$ & $\mathrm{CA}$ & MZUSP 66620 & São Domingos, GO \\
\hline \multicolumn{5}{|l|}{ Microhylidae } \\
\hline \multicolumn{5}{|l|}{ Chiasmocleis } \\
\hline Chiasmocleis centralis & $\mathrm{T}$ & $\mathrm{E}$ & MZUSP 7547 & Aruanã, GO \\
\hline Dermatonotus muelleri & $\mathrm{T}$ & $\mathrm{O}$ & CHUNB 44333 & Buritizeiro, MG \\
\hline $\begin{array}{l}\text { Elachistocleis bicolor } \\
\text { Elachistocleis }\end{array}$ & $\mathrm{T}$ & $S$ & CHUNB 49299 & Bonito, MS \\
\hline bumbameuboi* & $\mathrm{T}$ & $\mathrm{NE}$ & CHUNB 51588 & Carolina, MA \\
\hline $\begin{array}{l}\text { Elachistocleis cesarii* } \\
\text { Elachistocleis }\end{array}$ & $\mathrm{T}$ & W & CHUNB 48356 & Brasília, DF \\
\hline $\begin{array}{l}\text { matogrosso* } \\
\text { Elachistocleis }\end{array}$ & $M$ & $\mathrm{CHA}$ & MNRJ 4812 & Cuiabá, MT \\
\hline piauiensis* & $\mathrm{T}$ & $\mathrm{O}$ & MNRJ 4814 & Cuiabá, MT \\
\hline \multicolumn{5}{|l|}{ Pipidae } \\
\hline Рipa pipa* & M & AM & MNRJ 55664 & Bonópolis, GO \\
\hline \multicolumn{5}{|l|}{ Ranidae } \\
\hline Lithobates palmipes & $\mathrm{M}$ & AM & ZUFG 4727 & Piranhas, GO \\
\hline \multicolumn{5}{|l|}{ Strabomantidae } \\
\hline $\begin{array}{l}\text { Barycholos ternetzi } \\
\text { Oreobates }\end{array}$ & $\mathrm{T}$ & $E$ & CHUNB 39090 & Brasília, DF \\
\hline heterodactylus & $\mathrm{T}$ & $E$ & MNRJ 5089 & $\begin{array}{l}\text { Cáceres, MT } \\
\text { Chapada dos }\end{array}$ \\
\hline Pristimantis crepitans & $\mathrm{T}$ & $E$ & UFMT 1383 & $\begin{array}{l}\text { Guimarães, MT } \\
\text { Chapada dos }\end{array}$ \\
\hline $\begin{array}{l}\text { Pristimantis dundeei } \\
\text { Pristimantis aff. }\end{array}$ & $\mathrm{T}$ & $E$ & UFMT 7353 & Guimarães, MT \\
\hline $\begin{array}{l}\text { gutturalis* } \\
\text { Pristimantis aff. }\end{array}$ & $\mathrm{T}$ & $\mathrm{E}$ & CHUNB 51825 & Carolina, MA \\
\hline dundeei* ${ }^{*}$ & $\mathrm{~T}$ & $E$ & $n / d$ & Alto Araguaia, MT \\
\hline
\end{tabular}




\section{Capítulo 2}

\section{A historical perspective on the distribution of anuran amphibians in Brazilian Cerrado}

Valdujo, P. H. \& M. Martins. Manuscrito em preparação que será submetido ao periódico Journal of Biogeography 


\title{
A historical perspective on the distribution of anuran amphibians in Brazilian Cerrado
}

\begin{abstract}
Expanding temporal, spatial and phylogenetic scales help providing insights into the importance of environmental requirements and historical constrains on the assembly of local and regional communities. Here we analyze the distribution of anuran genera in the Brazilian Cerrado, regarding the relationship between the Cerrado and the adjoining forest domains, namely the Amazon and the Atlantic Forest. We use co-occurrence analyses and model selection to test for spatial structure in genera distribution and determine which factors are more likely to determine genera distribution: rainfall, topography or distance to the closest forest domain. Sixteen out of the 35 genera occurring in the Cerrado have their center of diversity in forest domains. Of these, five genera occur in the Amazon and the Cerrado, seven genera occur in the Atlantic Forest and the Cerrado. Some Cerrado anuran genera are distributed in a checkerboard pattern, and co-occur less than it would be expected by chance. However, when we analyze only the five Amazonian genera, we found no checkerboard pattern, and we obtained the same result for the eight Atlantic taxa. This spatial structure within the Cerrado is influenced by the interplay of environmental conditions and historical constrains: Atlantic genera are mostly distributed in mountainous and upland areas, with low precipitation and closer to the boundaries of Atlantic Forest, whereas Amazonian genera are distributed in valleys closer to the boundaries of the Amazon. Our results suggest that the heterogeneity in anuran distribution in the Cerrado may have a historical basis, which interact with present-day constraints, such as climate, habitat availability and ecological interactions, to shape local and regional assemblages.
\end{abstract}

\section{Introduction}

During the past two decades there has been a growing concern on the importance of large scale ecological and biogeographical processes, and their connections with contemporary processes to understand species diversity and distribution patterns (Ricklefs 1987; Ricklefs \& Schluter 1993; Wiens \& Donoghue 2004). It is desirable that temporal, spatial and phylogenetic 
scales are expanded in order to get insights into the importance of environmental requirements and historical constrains on the assembly of local and regional communities, and understand why the members of a clade have dispersed to some places and not to others (Wiens \& Donoghue 2004). Biotic dispersion leaves a significant historical imprint on the evolution of species assemblages, and coupled with subsequent vicariance, these two processes result in biotas that are highly structured historically (Cracraft 1994). Thus, similar geographic pattern exhibited by different clades may indicate historically structured processes rather than a result of long-distance dispersal (Wiley 1980; Cracraft 1994). In the absence of complete phylogenies, the origin of diversity patterns can be alternatively assessed through the analysis of diversity and composition at taxonomic levels more inclusive than species, such as genera or families (Cadle \& Greene 1993; Schluter \& Ricklefs 1993).

The spatial structure of regional communities reflects the history of taxa assembly. Although vicariance biogeography studies usually take for granted that there is a non-random structure in species distribution (Hausdorf \& Hennig 2003), tests of clustered distribution based on null models have been only recently proposed and improved (Hausdorf \& Hennig 2003; Mast \& Nyffeler 2003; Giokas \& Sfenthourakis 2008). Null models of species cooccurrence (Gotelli 2000) were adapted to a vicariance biogeography framework through some modifications in the basic assumptions. In this framework, null models are used to identify biotic elements that led to the definition of areas of endemism (Hausdorf \& Hennig 2003; Mast \& Nyffeler 2003; Giokas \& Sfenthourakis 2008). Here, we take the less restrictive assumptions of the vicariance biogeography framework, rather than the species interactions framework: assemblages are ecologically diverse and defined taxonomically, rather than based on predicted interactions. Furthermore, sites are environmentally heterogeneous and likely have different assemblages due to historical and environmental factors. We take advantage of the "dilution effect" of broad-scale spatial analysis (Diamond \& Gilpin 1982), which hampers the detection of species interactions. Hence, we can examine the imprint of historical and environmental constrains on spatial structure of taxa. 
Spatial patterns of species richness are explained by environmental and historical factors for many taxa in global (Buckley \& Jetz 2007), continental (Williams \& Hero 2001; Rahbek et al. 2007) and regional scales (Diniz-Filho et al. 2008; Hawkins 2010). The combined action of rainfall, temperature, and topographic gradients seems to be constrained by the zone of origin and dispersion ability of different clades to define richness and species composition (Ricklefs 2004; Wiens \& Donoghue 2004). Further, the hypotheses of phylogenetic niche conservatism predicts that related species have similar environmental requirements and tolerances (Wiens \& Graham 2005), providing an additional imprint of history on the spatial structure of regional assemblages. Specifically, large-scale studies of amphibian richness and distribution patterns described a remarkable importance of climatic and topographic gradients, constrained by historical factors (Buckley \& Jetz 2007), as well as the existence of phylogenetic signal in species climate niches (Hof et al. 2010).

Amphibians have, in general, a narrow but variable tolerance to environmental characteristics, especially those related to water loss (Schmid 1965; Wells 2007). However, many behavioral, physiological, and morphological adaptations, combined with the greatest diversity in reproductive modes among terrestrial vertebrates (Duellman \& Trueb 1986; Haddad \& Prado 2005) allow them to occur in various habitat types. The American tropics, especially rainforests, present the greatest diversity of anurans in the world. This diversity decreases from wetter to drier regions and from lower to higher elevations, and is also highly influenced by the history of family groups (Duellman 1988). Neotropical amphibians can be divided into two major ecological groupings: a larger group of species restricted to closed canopy forests and a smaller group of open formation species occurring in drier habitats, such as the Cerrado, Chaco and Caatinga domains (Heyer 1988).

Landscapes in the Cerrado are dominated by savannas and grasslands. However, gallery forest and patches of semideciduous and deciduous forests are frequent and widespread throughout the domain, representing an important connection with neighbor forest domains, as demonstrated for some groups of plants (Oliveira-Filho \& Ratter 1995), birds 
(Silva 1997), small mammals (Costa 2003), and sphingid moths (Amorim et al. 2009). Although the role of forest habitats for anuran distribution has never been systematically investigated, it is known that some species typical of forest domains may occur deep or marginally into the Cerrado, especially in gallery forests (Colli et al. 2002). Furthermore, the extensive contact zones between the Cerrado and the Amazon as well as the Cerrado and the Atlantic Forest may have allowed species interchange through time, influencing assembly history. However, amphibians tend to have a lower dispersion ability and a highly specific microhabitat requirements compared to other groups, which result in smaller ranges, greater potential for isolation, and therefore, a very high number of endemic species (Beebee \& Griffiths 2005). Hence, taking into account that (i) there are remarkable differences in environmental traits between the Cerrado and neighbor forest domains, and (ii) forest habitats within the Cerrado may preserve some of the structural features of forest domains, we can expect that the Cerrado may act either as a filter, preventing Atlantic Forest clades to contact Amazonian species, or as a corridor connecting these two faunal groups.

Anuran species in the Cerrado have a structured distribution, in which species shared with the Atlantic Forest are distributed in the southeastern part of the biome, whereas species shared with the Amazon Forest are distributed in the northwestern part (see Chapter 1). However, endemic species belonging to many different lineages are widespread throughout the domain, and their distribution patterns have never been analyzed within a historical framework. In order to investigate the relationship of Cerrado anurans with the Atlantic Forest and Amazonian fauna under a historical standpoint, we explored the distribution of anuran lineages, rather than species, within the Cerrado. In the absence of complete phylogenies including a substantial number of Cerrado taxa, we attempted to retrieve historical biogeographical patterns through the analysis of genera distribution, assuming their internal monophyly. We specifically addressed the questions that follow: (1) Is there spatial structure in co-occurrence patterns among anuran genera in Cerrado? (2) To what extent environmental gradients and historical constrains explain distribution patterns of genera within the Cerrado? 


\section{Methods}

Study area

The Cerrado domain covers the sedimentary and crystalline plateau of central Brazil and harbors the watershed of three of the largest river basins in South America: Amazon, Paraguay and São Francisco (Brasil \& Alvarenga 1989). Together with Caatinga and Chaco, it forms the diagonal of open formations (Vanzolini 1963), which lies between the Amazon and the Atlantic Forest. The climate is characterized by a rainy season from October to April and a marked dry season from May to September (Nimer 1979). Transition zones between the Cerrado and neighbor domains are sometimes wide and have vegetation traits from both domains, what make it difficult to precisely define its boundaries. We adopted a geographic, rather than physiognomic criteria to delimit the Cerrado domain from the official Brazilian vegetation map (Veloso et al. 1991). We selected all vegetation types included in the savanna class, as well as transition zones between savanna and any other vegetation class that were continuously distributed. Patches of deciduous and semideciduous forest located within the boundaries of the delimited area were also included. We decided not to include the savanna enclaves of Amazonia, Atlantic Forest or Caatinga, neither the Pantanal floodplains, because we considered that these areas have unique histories that must be analyzed in a more specific context.

\section{Data collection}

We built a database on anuran distribution in the Brazilian Cerrado from museum specimens, taxonomic literature records, and field surveys. We examined 31,024 specimens collected in Cerrado localities and deposited in the following zoological collections: Museu de Zoologia da Universidade de São Paulo (MZUSP), Museu Nacional do Rio de Janeiro (MNRJ), Coleção Herpetológica da Universidade de Brasília (CHUNB), Coleção Herpetológica da Universidade Federal de Goiás (ZUFG), Museu Paraense Emílio Goeldi (MPEG), Coleção Zoológica da Universidade Federal do Mato Grosso (ZUFMT), Coleção Zoológica da Universidade Federal do Mato Grosso do Sul (ZUFMS), Museu de Zoologia Adão Cardoso - Universidade Estadual de Campinas (ZUEC), Coleção Célio Haddad - Universidade Estadual Paulista, 
Campus Rio Claro (CFBH), Coleção Herpetológica do Museu de Ciências Naturais da PUC-Minas (MCN), Coleção do Departamento de Zoologia e Botânica (DZSJRP) housed at UNESP, São José do Rio Preto. We also performed fieldwork in several Cerrado localities in the states of Mato Grosso, Paraná, Bahia, Tocantins and Maranhão from 2007 to 2009. Species identities were assessed through comparison with type specimens whenever possible and taxonomic literature. For each examined specimen we recorded the complete available collecting data.

From this dataset, we selected well-sampled localities, from which at least 20 species were recorded and built a matrix in which lines represent localities and columns represent genera. Each cell was filled either with 1, for presence, 0 for absence. We then classified all genera into one of four biogeographic groups according to their center of diversity (i. e. the region where each genus is more diversified): i) Atlantic group: genera which have the majority of its species distributed in the Atlantic Forest, and few in the Cerrado; ii) Amazon group: genera which have the majority of its species distributed in the Amazon, and few in the Cerrado; iii) open domain group: genera that are more diversified in the Cerrado, Chaco and/or Caatinga; iii) widespread group: genera that are highly diversified in more than one domain. We compiled distribution data for species included in our analyses that do not occur in the Cerrado from Frost (2010), IUCN (2010), SBH (2010) and specific literature for each of the taxa.

We used three environmental and two historical predictors to analyze distribution patterns of Cerrado anuran genera (table 1). We downloaded annual precipitation grid map from WorldClim (Hijmans et al. 2005; available from http://www.worldclim.org/), and elevation grid map from EROS-USGS (U.S. Geological Survey Center for Earth Resources Observation and Science; http://eros.usgs.gov/products/elevation/hydro1k.html). From these, we calculated the mean annual precipitation, mean elevation and elevation range for a $20 \mathrm{Km}$ buffer around each sampling site. This procedure was necessary to overcome the bias induced by the use of municipality centroid coordinates to represent sampling sites. To evaluate the outcomes of dispersion constrains we measured the shorter distance between each point and each forest domain, namely Atlantic Forest and Amazon. We decided not 
to include temperature as a predictor due to its high correlation with elevation $(r>0.8)$, which would not allow us to untangle the effects of these two variables. Therefore, we assume that spatial patterns predicted by elevation may be an outcome of the elevation gradient per se, of the temperature gradient, of the sum or the interaction between these two factors.

Table1. Environmental predictors included in the GLMs and the justification for inclusion of each predictor.

\begin{tabular}{ll}
\hline Predictor & Justification for predictor \\
\hline Mean elevation & Since Amazon covers mostly lowlands and Atlantic Forest \\
& cover mostly mountains and uplands, Amazon lineages \\
& are expected to occur at lower elevations, whereas \\
& Atlantic lineages are expected to occur at higher \\
& elevations in the Cerrado \\
& Since the Amazon covers mostly lowlands and Atlantic \\
& Forests covers mostly mountains, Amazonian lineages \\
Elevation range & are expected to occur in lower elevation ranges, whereas \\
& Atlantic lineages are expected to occur at higher elevation \\
& ranges in the Cerrado \\
Both Amazonian and Atlantic lineages area expected to & respond positively to annual precipitation, since many \\
anurans rely on temporary water bodies to breed. & Genera more diversified in the Atlantic Forest are \\
precipitation & expected to be distributed within the Cerrado in areas that \\
are closer to the contact with Atlantic Forest \\
Distance to Atlantic
\end{tabular}

Analysis

i. Co-occurrence

We analyzed genera co-occurrence for Amazonian, Atlantic and open domains through a null model analysis (Gotelli 2000), on a presence/absence 
matrix where lines represented 86 well sampled localities and columns represented genera that contained at least one Cerrado species. We performed a Monte Carlo randomization of observed data in EcoSim v. 7.7.2 (Gotelli \& Entsminger 2010), using the following configuration: fixed rows and columns and swap algorithm. We calculated C-score indices for observed data and for each randomization. We then compared the C-score obtained from the observed data to the distribution of values of $\mathrm{C}$-score obtained from 5000 randomizations of the presence-absence matrix. In order to look for any historical signature in co-occurrence patterns, after running the co-occurrence analysis for all genera together, we repeated the procedure for four groups of genera based on their center of diversity: Atlantic genera, Amazonian genera, open domain genera and widespread genera. For the last group we removed taxa occurring in all 86 localities, to enhance our ability to detect a possible checkerboard pattern among the remaining species.

\section{ii. Distribution of genera}

To test for the importance of environmental and historical factors on anuran genera distribution within the Cerrado, we built generalized linear models (GLMs) with binomial errors (logistic regression) for the two geographic groupings of genera. Hence, one series of models were made for genera more diversified in the Amazon and another series for genera more diversified in the Atlantic Forest. We used mean elevation, elevation range, precipitation and distance to the Atlantic Forest or Amazon, as well as the interaction between each pair of variables as predictors, and the presence or absence of pooled geographic grouping of genera as response variable. We selected the best fitted model among all possible combinations using the Akaike information criteria corrected for small samples (AICc), calculated in Multi Model Inference package for R (Burnham \& Anderson 2002). We examined and considered equally likely all models with dAIC lower than 2 (Bolker, 2008). We used weighted AICc and model-averaging procedure to evaluate the relative importance of all variables included in the top models (Burnham \& Anderson 2002). 


\section{Results}

Sixteen out of the 35 genera occurring in the Cerrado have their center of diversity in forest domains (table 2). Of these, five genera occur in the Amazon and the Cerrado, seven genera occur in the Atlantic Forest and the Cerrado, whereas Allobates and Vitreorana are widespread both in the Amazon and the Atlantic Forest, and also occur in a few localities within the Cerrado. Six genera have their center of diversity in the diagonal of open domains of South America, occurring in the Cerrado and the Chaco and/or the Caatinga. Thirteen genera are widespread in South America and we were not able to determine a center of diversity for them because they are either well diversified in more than one domain (e.g. Scinax, Hypsiboas) or they are represented by one or few species in each domain (e.g. Ceratophrys, Barycholos). Some Cerrado anuran genera are distributed in a checkerboard pattern, and co-occur less than it would be expected by chance (table 3, figure 1). However, when we analyze only the five Amazonian genera, we found no checkerboard pattern, and we obtained the same result for the eight Atlantic taxa (table 3).

Table 2. Cerrado anuran genera grouped by their center of diversity.

\begin{tabular}{|c|c|}
\hline $\begin{array}{l}\text { Center of } \\
\text { Diversity }\end{array}$ & Genera \\
\hline Atlantic Forest & $\begin{array}{l}\text { Aplastodiscus, Bokermannohyla, Crossodactylus, Hylodes, } \\
\text { Ischocnema, Phasmahyla, Thoropa }\end{array}$ \\
\hline Amazonia & $\begin{array}{l}\text { Adelphobates, Oreobates, Osteocephalus, Pristimantis, } \\
\text { Rhaebo }\end{array}$ \\
\hline Open Domains & $\begin{array}{l}\text { Corythomantis, Dermatonotus, Eupemphix, Odontophrynus, } \\
\text { Pleurodema, Pseudopaludicola }\end{array}$ \\
\hline Widespread & $\begin{array}{l}\text { Allobates, Ceratophrys, Chiasmocleis, Dendropsophus, } \\
\text { Elachistocleis, Hypsiboas, Leptodactylus, Phyllomedusa, } \\
\text { Physalaemus, Proceratophrys, Pseudis, Rhinella, Scinax, } \\
\text { Trachycephalus }\end{array}$ \\
\hline Andes & Ameerega, Barycholos \\
\hline
\end{tabular}


Table 3. Observed and simulated values of C-score, variance of simulated values and $p$-value representing the comparison of the presence absence matrix versus the null model. The analysis was run three times, first on all genera, than only on Atlantic genera and finally only on Amazonian genera.

\begin{tabular}{llll}
\hline & All genera & Atlantic only & Amazonian only \\
\hline Observed C-score & 73.72 & 24.04 & 36.10 \\
Mean simulated C-score & 69.10 & 23.61 & 34.93 \\
Variance of simulated C-score & 0.22 & 0.51 & 1.02 \\
p-value & $<<0.01$ & 0.24 & 0.13 \\
\hline
\end{tabular}

Atlantic and Amazonian taxa responded differently to environmental variables, and both responded to the distance to the contact zone with the forest domain they are more related to. Based on the generalized linear model on Atlantic genera, we selected four top models to explain their distribution within the Cerrado ( $\mathrm{AAICc}<2)$, which had a summed weight of 0.71 (table 4). Species belonging to Atlantic genera are more likely to be found in areas closer to the contact zone with the Atlantic Forest, in sites where mean elevation and elevation range are higher and annual rainfall is lower (table 5). Similarly, the generalized linear models on Amazonian genera also resulted in four top models to explain their distribution within the Cerrado, which had a summed weight of 0.63 (table 6). Species belonging to Amazonian genera are more likely to be found in areas closer to the contact zone with Amazon, in sites where mean elevation is lower and elevation range is higher (table 7 ).

Table 4. Four top models $(\mathrm{dAIC}<2)$ explaining the relationship of the presence of Atlantic genera as a function of the environmental and historical predictors.

\begin{tabular}{llll}
\hline Model & AICc & dAICc & weight \\
\hline Rain + Elev_range + Elev_mean + dist_Atl & 69.78 & 0 & 0.26 \\
Rain + Elev_range + dist_Atl & 70.55 & 0.77 & 0.18 \\
Rain + Elev_range + Elev_mean & 70.65 & 0.87 & 0.17 \\
Elev_range + dist_Atl & 71.70 & 1.91 & 0.10 \\
\hline
\end{tabular}


Table 5. Parameter estimates based on top models explaining occurrence of Atlantic Forest genera using model-averaging procedure (Burnham \& Anderson 2002).

\begin{tabular}{llll}
\hline Parameter & Coefficient & Variance & Relative Importance \\
\hline Intercept & 2.07 & $1.52 \times 10^{3}$ & - \\
Elevation range & $8.02 \times 10^{-11}$ & $3.32 \times 10^{-11}$ & 1.00 \\
Annual rainfall & $-4.87 \times 10^{-3}$ & $5.41 \times 10^{-10}$ & 0.84 \\
Distance to Atlantic Forest & $-3.01 \times 10^{-6}$ & $5.13 \times 10^{-23}$ & 0.78 \\
Mean elevation & $1.89 \times 10-3$ & $4.78 \times 10-9$ & 0.72 \\
\hline
\end{tabular}

Table 6. Four top models $(\mathrm{dAIC}<2)$ explaining the relationship of the presence of Amazonian genera as a function of the environmental and historical predictors.

\begin{tabular}{llll}
\hline Model & AICc & dAICc & weight \\
\hline Elev_range + Elev_mean + dist_Amaz & 76.77 & 0 & 0.22 \\
Elev_range + Elev_mean + dist_Amaz + & 77.21 & 0.44 & 0.18 \\
Elev_mean * dist_Amaz & & & \\
Elev_mean + dist_Amaz & 77.87 & 1.10 & 0.13 \\
Elev_mean + dist_Amaz * Elev_mean * & 78.32 & 1.55 & 0.10 \\
dist_Amaz & & & \\
\hline
\end{tabular}

Table 7. Parameter estimates based on top models explaining occurrence of Amazonian genera using model-averaging procedure (Burnham \& Anderson 2002).

\begin{tabular}{llll}
\hline Parameter & Coefficient & Variance & Relative Importance \\
\hline Intercept & 2.01 & $8.05 \times 10^{1}$ & - \\
Distance to Amazon & $-2.83 \times 10^{-6}$ & $4.94 \times 10^{-23}$ & 1.00 \\
Mean elevation & $-2.65 \times 10^{-3}$ & $8.24 \times 10^{-10}$ & 0.94 \\
Elevation range & $1.88 \times 10^{-3}$ & $2.31 \times 10^{-11}$ & 0.57 \\
Dist_Amazon *Elev_mean & $-2.79 \times 10^{-9}$ & $7.06 \times 10^{-34}$ & 0.38
\end{tabular}




\section{Discussion}

Understanding biogeographical and environmental factors that influence species distribution provides insight into the history of species assembly. Here we used a null model approach (Gotelli 2000) to test for spatial structure and a model selection approach (Bolker 2008) to identify historical and environmental factors that best explain anuran genera distribution patterns, grouped by their center of diversity (the phytogeographical domain where e a genus most diversified). Anuran genera are distributed in a checkerboard pattern within the Cerrado, due to the nearly mutually exclusive distribution of genera most diversified in the Atlantic Forest, compared to those most diversified in the Amazon. This spatial structure within the Cerrado is influenced by the interplay of environmental conditions and historical constrains, since Atlantic genera are distributed in mostly in mountainous and upland areas, with low precipitation and closer to the boundaries of Atlantic Forest, whereas Amazonian genera are distributed in valleys closer to the boundaries of the Amazon. These results suggest that limiting environmental conditions and/or unsuitable habitats of the plateaus in central Cerrado act as a barrier that prevents overlap in the distribution of these two biogeographic groups, though allowing overlap of taxa within biogeographic groups.

Anuran genera co-occur less than it would be expected by chance within the Cerrado. Although neither open-domain nor widespread genera are spatially structured in the studied scale, this result is due to the nearly mutually exclusive distribution of Amazonian and Atlantic genera, which form an almost perfect checkerboard pattern. The absence of an overlap in the distribution of Amazonian and Atlantic lineages suggests that, at least for anurans, gallery forests in the Cerrado do not promote species interchange between the two forest domains. This pattern differs from that described for small mammals (Costa 2003) and woody plants (Oliveira-Filho \& Ratter 1995), for which gallery forests work as corridors connecting the two forest domains, and promoting biotic exchanges. However, our results are consistent with the distribution of Amazonian and Atlantic bird species that extend their range into the Cerrado gallery forests, which present a highly 
structured distribution pattern (Silva 1996). Furthermore, different environmental factors seem to be more important in determining the distribution of taxa belonging to each biogeographic group, indicating that different ecophysiological requirements and restrictions prevail, rather than interspecific interactions. Atlantic taxa extend their range along mountains and seasonally dry or semideciduous forests in the eastern and central parts of the domain. Their deeper distribution, compared to Amazonian taxa, is also consistent to the pattern described for birds (Silva 1996).

The occurrence of Atlantic genera in the Espinhaço range and other mountains in southeastern and central Cerrado explains the importance of the mean elevation and elevation range in models selected to describe the distribution of Atlantic genera. Connections of the Espinhaço Range with the Atlantic Forest are evident from shared species and lineages. Moreover, the relationship of species and lineages occurring on the highlands of Central Cerrado with species from the Espinhaço Range was described as one of the remarkable patterns of disjunct distribution for plants (Giulietti \& Pirani 1988). The shared flora was suggested to reflect the similar geological, physical and climatic features of these regions, and the probable links among highland floras in the past (Giulietti \& Pirani 1988). For instance, the distribution pattern of the genus Bokermannohyla in Cerrado is similar to those of many plant lineages typical of highlands, such as Lychnophora ericoides (Asteraceae; Collevatti et al. 2009) and the genus Diplusodon (Lythraceae; Giulietti \& Pirani 1988). Furthermore, four out of seven Atlantic genera of anurans in the Cerrado occur only on the highlands of Espinhaço, close to the boundary of the domain and its contact with Atlantic Forest (Crossodactylus, Hylodes, Phasmahyla, Thoropa). On the other hand, Ischnocnema extends its distribution to the seasonal dry forests of São Francisco River Basin and Tocantins River basin, and Bokermannohyla and Aplastodiscus can be found respectively in highlands and in gallery forests of Central Plateau (figure 1).

Amazonian taxa are more likely to occur in lowlands closer to the boundaries of the Cerrado and contact with the Amazon. Amazonian lineages in Cerrado are highly associated to the Araguaia and Tocantins river valleys, which belong to the Amazon basin. The Amazonian genera Osteocephalus, Adelphobates, Rhaebo, and Pristimantis co-occur in lowland forests. 
Amazonian lineages extend their range southward to the upper Paraguay River basin, on the western border of Pantanal. This pattern is consistent with that described for reptiles and birds (Junk et al. 2006). Although gallery forests do not act as corridors for anurans as they do for small mammals and woody plants (Oliveira-Filho \& Ratter 1995; Costa 2003), they are very important for the distribution of lowland Amazonian lineages in Cerrado. Gallery forests allow these taxa to extend their range as south as the mid Tocantins River (e.g. Adelphobates; Pavan \& Dixo 2004), or even deeper in upper Tocantins River (Pristimantis and Rhaebo). These localities lie in core Cerrado and perhaps this pattern reflects a greater importance of the drainage network over climatic or phytophysionomic traits for the distribution of some species. Thus gallery forests may act as corridors promoting the dispersal of some Amazonian components, and phylogeographic studies are needed in order to test this hypothesis.

The distribution pattern that we found suggests that Cerrado representatives of genera more diversified in forest domains partially conserve the climatic and habitat requirements of their forest relatives, in spite of the lower availability of this kind of habitat. The climatic niche conservatism (Wiens \& Graham 2005) is probably an outcome of the combination of physiological constrains and breeding habitat requirements, that ends up restricting Amazonian taxa to the lowlands and Atlantic taxa to mountains. For instance, many Atlantic elements that inhabit the Cerrado breed exclusively in temporary mountain streams (e.g. Bokermannohyla, Crossodactylus, Hylodes, Phasmahyla; Eterovick \& Sazima 2000; Haddad \& Prado 2005; Leite et al. 2008). This kind of habitat is largely available in Atlantic Forest, where these genera are more diversified, but not in the Cerrado. These differences in habitat availability might explain why these genera have a more restricted distribution in the Cerrado compared to the Atlantic Forest. Similarly, Amazonian taxa are more associated to lowlands in the Cerrado, where gallery forests are moister and wider and both habitat structure and climate are more similar to the eastern portion of the Amazon. It is remarkable that species belonging to forest genera have greater similarity in life history traits and environmental requirements with their relatives that are distributed in different phytogeographical domains than they have with other 
species occurring in the same regions, that have different biogeographical histories. The conservation of traits and requirements in a different and probably less suitable habitat likely explains the lower diversity of these genera in the Cerrado, compared to the Atlantic Forest and the Amazon. This pattern is consistent with the evolutionary model that states that gradients of diversity will have more species in environments that are older, more widespread or less stressful (Ricklefs 2006). Although we cannot assure the relative ages of phytogeographical domains in South America, the habitats of these lineages are undoubtedly more stable and widespread in Amazon and Atlantic Forest, compared to the Cerrado.

Null models of co-occurrence (Gotelli 2000) have been used in a historical framework to identify biotic elements and areas of endemism (Mast \& Nyffeler 2003; Giokas \& Sfenthourakis 2008). Here we propose that this method can be also useful to identify historical connections between different phytogeographical domains through the analysis of the distribution of genera, rather than species. Due to the nature of this system and to the spatial scale that we adopted, phylogenetic signal was so strong that could be retrieved from a simple taxonomic arrangement, in the absence of complete phylogenies for all lineages. This reinforces the idea that historical constrains can be retrieved from good taxonomic hierarchical structures (e.g. Cadle \& Greene 1993). However we also state that, although this framework was helpful for a first approach, using phylogenies and phylogenetic structure of communities will greatly improve the results and their interpretation. We will than be able to include additional taxa, as well as identify speciation modes and more detailed biogeographic relationship between areas (McPeek \& Brown 2000). This reinforces the importance of expanding both spatial and phylogenetic (or, alternatively, taxonomic) scales to access the history of species assembly (Wiens \& Donoghue 2004).

We propose that the heterogeneity in anuran distribution in the Cerrado may have a historical basis, which interact with present-day constraints (such as climate, habitat availability and ecological interactions) to shape local and regional assemblages. Future studies focusing on the relationship between local and regional assemblages in this system should take into account the biogeographical barriers inside the domain to define the regional species pool. 
Taxonomic studies on Cerrado lineages and the inclusion of endemic species in phylogenies are urgently needed to further clarify biogeographic patterns and processes.

\section{References}

Amorim, F. W., R. S. de-Ávila-Jr, A. J. A. de-Camargo, A. L. Vieira \& P. E. Oliveira (2009). A hawkmoth crossroads? Species richness, seasonality and biogeographical affinities of Sphingidae in a Brazilian Cerrado. Journal of Biogeography 36: 662-674.

Beebee, T. J. C. \& R. A. Griffiths (2005). The amphibian decline crisis: a watershed for conservation biology? Biological Conservation 125: 271285.

Bolker, B. M. (2008). Ecological Models and Data in R. Princeton, Princeton University Press.

Brasil, A. E. \& S. M. Alvarenga (1989). Relevo. Geografia do Brasil: Região Centro-Oeste. A. C. Duarte. Rio de Janeiro, FIBGE - Diretoria de Geociências: 53-72.

Buckley, L. B. \& W. Jetz (2007). Environmental and historical constraints on global patterns of amphibian richness. Proceedings of the Royal Society B: Biological Sciences 274: 1167-1173.

Cadle, J. E. \& H. W. Greene (1993). Phylogenetic patterns, biogeography, and the ecological structure of neotropical snake assemblages.

Species diversity in ecological communities, historical and geographical perspectives. R. E. Ricklefs and D. Schluter. Chicago, The University of Chicago Press: 281-293.

Collevatti, R. G., S. G. Rabelo \& R. F. Vieira (2009). Phylogeography and disjunct distribution in Lychnophora ericoides (Asteraceae), an endangered cerrado shrub species. Annals of Botany 104: 655-664.

Colli, G. R., R. P. Bastos \& A. A. Araujo (2002). The Character and Dynamics of the Cerrado Herpetofauna. The Cerrados of Brazil: ecology and natural history of a neotropical savanna. P. S. Oliveira and R. J. Marquis. New York, Columbia University Press: 223-239.

Costa, L. P. (2003). The historical bridge between the Amazon and the Atlantic Forest of Brazil: a study of molecular phylogeography with small mammals. Journal of Biogeography 30: 71-86.

Cracraft, J. (1994). Species Diversity, Biogeography, and the Evolution of Biotas. American Zoologist 34: 33-47.

Diamond, J. \& M. Gilpin (1982). Examination of the Null Model of Connor and Simberloff for Species Co-Occurrences on Islands. Oecologia 52: 6474.

Diniz-Filho, J. A. F., L. M. Bini, C. M. Vieira, D. Blamires, L. Terribile, R. Bastos, G. de Oliveira \& B. Barreto (2008). Spatial patterns of terrestrial vertebrate species richness in the Brazilian Cerrado. Zoological Studies 47: 146-157.

Duellman, W. E. \& L. Trueb (1986). Biology of Amphibians. New York, McGraw-Hill Publishing Company. 
Duellman, W. E. (1988). Patterns of species diversity in anuran amphibians in the American tropics. Annals of the Missouri Botanical Garden 75: 79104.

Eterovick, P. C. \& I. Sazima (2000). Structure of an anuran community in a montane meadow in southeastern Brazil: effects of seasonality, habitat, and predation. Amphibia-Reptilia 21: 439-461.

Frost, D. (2010). Amphibian Species of the World: an Online Reference. Version 5.4 - Electronic Database accessible at http://research.amnh.org/vz/herpetology/amphibia/. Retrieved 18 January 2011, 2011.

Giokas, S. \& S. Sfenthourakis (2008). An improved method for the identification of areas of endemism using species co-occurrences. Journal of Biogeography 35: 893-902.

Giulietti, A. M. \& J. R. Pirani (1988). Patterns of geographic distribution of some plant species from the Espinhaco range, Minas Gerais and Bahia, Brazil. Workshop on Neotropical Distribution Patterns Rio De Janeiro, Academia Brasileira de Ciências.

Gotelli, N. J. (2000). Null model analysis of species co-occurrence patterns. Ecology. 81: 2606-2621.

Gotelli, N. J. \& G. Entsminger (2010). EcoSim: Null models software for ecology. . Jericho, Acquired Intelligence Inc. \& Kesey-Bear.

Haddad, C. F. B. \& C. P. A. Prado (2005). Reproductive modes in frogs and their unexpected diversity in the Atlantic Forest of Brazil. BioScience 55: 207-217.

Hausdorf, B. \& C. Hennig (2003). Biotic element analysis in biogeography. Systematic Biology 52: 717-723.

Hawkins, B. A. (2010). Multiregional comparison of the ecological and phylogenetic structure of butterfly species richness gradients. Journal of Biogeography 37: 647-656.

Heyer, W. R. (1988). On frog distribution patterns east of the Andes. Proceedings of a Workshop on Neotropical Distribution Patterns.

Hijmans, R. J., S. E. Cameron, J. L. Parra, P. G. Jones \& A. Jarvis (2005). Very high resolution interpolated climate surfaces for global land areas. International Journal of Climatology 25: 1965-1978.

Hof, C., C. Rahbek \& M. B. Araújo (2010). Phylogenetic signals in the climatic niches of the world's amphibians. Ecography 33: 242-250.

IUCN (2010). IUCN Red List of Threatened Species. Version 2010.4. <http://www.iucnredlist.org>. Retrieved 3 January 2011, 2011.

Junk, W. J., C. N. da-Cunha, K. M. Wantzen, P. Petermann, C. Strussmann, M. I. Marques \& J. Adis (2006). Biodiversity and its conservation in the Pantanal of Mato Grosso, Brazil. Aquatic Sciences 68: 278-309.

Leite, F. S. F., F. A. Juncá \& P. C. Eterovick (2008). Status do conhecimento, endemismo e conservação de anfíbios anuros da Cadeia do Espinhaço, Brasil. Megadiversidade 4: 182-200.

Mast, A. R. \& R. Nyffeler (2003). Using a null model to recognize significant co-occurrence prior to identifying candidate areas of endemism. Systematic Biology. 52: 271-280.

McPeek, M. A. \& J. M. Brown (2000). Building a regional species pool: diversification of the Enallagma damselflies in eastern North America. Ecology 81: 904-920. 
Nimer, E. (1979). Climatologia do Brasil. Rio de Janeiro, Instituto Brasileiro de Geografia e Estatística.

Oliveira-Filho, A. T. \& J. A. Ratter (1995). A study of the origin of central Brazilian forests by the analysis of plant species distribution patterns. Edinburgh Journal of Botany 52: 141-194.

Pavan, D. \& M. Dixo (2004). A herpetofauna da área de influência do reservatório da Usina Hidrelétrica Luís Eduardo Magalhães. Humanitas 4: 13-30.

Rahbek, C., N. J. Gotelli, R. K. Colwell, G. L. Entsminger, T. F. L. V. B. Rangel \& G. R. Graves (2007). Predicting continental-scale patterns of bird species richness with spatially explicit models. Proceedings of the Royal Society B: Biological Sciences 274: 165-174.

Ricklefs, R. E. (1987). Community diversity - relative roles of local and regional processes. Science 235: 167-171.

Ricklefs, R. E. \& D. Schluter (1993). Species Diversity in Ecological Communities, Historical and Geographical Perspectives. Chicago, The University of Chicago Press.

Ricklefs, R. E. (2004). A comprehensive framework for global patterns in biodiversity. Ecology Letters 7: 1-15.

Ricklefs, R. E. (2006). Evolutionary diversification and the origin of the diversity-environment relationship. Ecology 87: S3-S13.

SBH (2010). Brazilian amphibians - List of species. Accessible at http://www.sbherpetologia.org.br. Retrieved 18 January 2011, 2011.

Schluter, D. \& R. E. Ricklefs (1993). Convergence and the regional component of species diversity. Species diversity in ecological communities: Historical and geographical perspectives R. E. Ricklefs and D. Schluter. Chicago, The University of Chicago Press: 230-240.

Schmid, W. D. (1965). Some aspects of the water economies of nine species of amphibians. Ecology 46: 261-269.

Silva, J. M. C. (1996). Distribution of Amazonian and Atlantic Forest Birds in Gallery Forests of the Cerrado Region, South America. Ornitologia Neotropical 7: 1-18.

Silva, J. M. C. (1997). Endemic bird species and conservation in the Cerrado Region, South America. Biodiversity and Conservation. 6: 435-450.

Vanzolini, P. E. (1963). Problemas faunísticos do Cerrado. Simpósio sobre o Cerrado. M. Ferri. São Paulo, Universidade de São Paulo: 307-320.

Veloso, H. P., A. L. R. Rangel-Filho \& J. C. Lima (1991). Classificação da vegetação brasileira, adaptada a um sistema universal. Rio de Janeiro, Fundação Instituto Brasileiro de Geografia e Estatística.

Wells, K. D. (2007). The Ecology and Behavior of Amphibians. Chicago and London, The University of Chicago Press.

Wiens, J. J. \& M. J. Donoghue (2004). Historical biogeography, ecology and species richness. Trends in Ecology \& Evolution 19: 639-644.

Wiens, J. J. \& C. H. Graham (2005). Niche conservatism: integrating evolution, ecology, and conservation biology. Annual Review of Ecology Evolution and Systematics 36: 519-539.

Wiley, E. O. (1980). Phylogenetic systematics and vicariance biogeography. Systematic Botany: 194-220.

Williams, S. E. \& J.-M. Hero (2001). Multiple determinants of Australian tropical frog biodiversity. Biological Conservation 98: 1-10. 


\section{Capítulo 3}

\section{Environmental and historical correlates of anuran beta diversity in Brazilian Cerrado}

Valdujo, P. H., C. H. Graham \& A. C. Carnaval. Manuscrito em preparação que será submetido ao periódico Ecography 


\title{
Environmental and historical correlates of anuran beta-diversity in Brazilian Cerrado
}

\begin{abstract}
Environmental conditions can inform the processes that underlie changes in species composition among local species assemblages. These factors operate at both local and broader spatial and temporal scales, assuming that species are best adapted to the environmental conditions where they evolved, and transition to other conditions requires evolutionary change. We built on recent advances in beta diversity studies to evaluate whether a historical approach can further our understanding about how evolutionary biogeographic processes impact patterns of beta diversity. We used the generalized dissimilarity modeling (GDM) approach to model anuran species compositional dissimilarity as a function of local and regional environmental conditions, as well as geographical separation in Brazilian Cerrado. We ran the same analysis on four combinations of two groups of predictors and two groups of response variables: local only or local and regional predictors, all species or endemics only. Patterns of beta diversity in the Brazilian Cerrado appear to be strongly influenced by the environmental gradients, since half of the dissimilarity in species composition was explained by the GDMs in the four models. Elevation was one of the most important variables in all models. Endemic species responded to regional environmental conditions stronger than all species. Endemics may be responding to environmental conditions based on how similar they are to the conditions of adjoining phytogeographical domains, since their sisterspecies are mostly distributed in these regions. Our results reinforce the importance of taking biogeographical history into account when analyzing spatial patterns of species diversity at a regional scale.
\end{abstract}

\section{Introduction}

Studies of beta diversity, the dissimilarity in species composition across sites (Whittaker 1960), can reveal the evolutionary and ecological processes underlying richness and distribution patterns. Recent research programs have amply addressed how species dissimilarity is influenced by contemporary processes, namely biological interactions and the interface 
between the physiological requirements of species and local environmental conditions such as climate (Azeria et al. 2009; Overton et al. 2009; Ashcroft et al. 2010), topography (Jankowski et al. 2009; Qian 2009), and microhabitat availability (Ellwood et al. 2009; Vasconcelos et al. 2009). Yet, environmental variables at broader spatial and temporal scales can also inform the processes that underlie the composition of local species assemblages - a topic not fully explored to date. For instance, assuming that species are best adapted to the environmental conditions where they evolved, and transition to other conditions requires evolutionary change (Ricklefs 2006), therefore, we expect to see a historical imprint in beta-diversity across large-scale environmental gradients. Building on recent advances in beta diversity studies afforded by generalized dissimilarity modeling (GDM, Ferrier et al. 2007), we evaluate whether a historical approach can further our understanding about how evolutionary biogeographic processes impact patterns of beta diversity.

The biogeographic history and environmental requirements of the regional species pool are expected to play a crucial role in beta diversity patterns along a large-scale environmental gradient (Wiens \& Donoghue 2004). Related species are more likely to have similar environmental requirements because they descend from a common ancestor (Brooks \& McLennan 1991). Therefore, if these requirements constrain taxa to exist along a portion of the environmental gradient, this indicates that phylogenetic niche conservatism likely influences patterns of dissimilarity. Often lineages within a given region originate outside the region and may be restricted to areas that are environmentally similar to where they originated (Wiens \& Graham 2005). If this is true, a historical imprint will be evident not only in recently dispersed species, but also in more ancient endemic species related to lineages that originated in other regions. If physiological and life history constraints are phylogenetically conserved, the interaction between the biogeographic history of the regional species pool and the environmental conditions within a study region will ultimately define and limit ranges of local species, and hence species assemblages.

Generalized dissimilarity modeling was developed to model species compositional dissimilarity as a function of local environmental conditions and 
geographical separation, by maximizing the correlation between environmental and biological dissimilarities. The resulting function can be used to subdivide a large study area into pre-defined numbers of more homogeneous landscapes (Ferrier 2002). The GDM has been increasingly applied in conservation-related studies such as dividing a region into landscapes with homogeneous communities or genetic diversity and environmental conditions in order to direct future sampling efforts and improve survey efficiency (Ashcroft et al. 2010) or to assess the representativeness of a reserve network (Overton et al. 2009; Thomassen et al. 2010). The GDM has been also applied to model intra-specific phenotypic and genetic variation as a function of environmental gradients, distance and a geographic barrier, in order to evaluate the evolutionary processes underlying generation and maintenance of biodiversity (Thomassen et al. 2010). These recent studies demonstrated that the GDM may be useful in both applied conservation and evolutionary frameworks. Here we expand its use to evaluate contemporary and historical factors influencing compositional change of anurofauna the complex biodiverse Brazilian Cerrado.

We use the anurofauna of the Brazilian Cerrado as a model system. Distributed in central Brazil, the Cerrado is a savanna phytogeographical domain adjacent to two South American forest biomes (Amazon and the Brazilian Atlantic rainforest) and three open domains (Caatinga, Pantanal and Chaco) through wide ecotones that favor biological interchange. Not surprisingly, communities within the Cerrado are structured as a mosaic, seemingly assembled from its multiple adjoining regional species pools as evidenced by the distribution patterns of anuran (Chapter two) woody plants (Oliveira-Filho \& Ratter 1995), birds (Silva 1996), and mammals (Costa 2003). This research suggests that the open interfluvial habitats within the Cerrado, especially the headwaters, act as barriers or filters limiting species dispersal and preserving strong signatures of the regional biogeographical history (see chapter one and chapter two). Because amphibian distribution within the Cerrado is strongly influenced by climatic and topographic factors (Eterovick \& Fernandes 2002; Diniz-Filho et al. 2008), environmental gradients are expected to influence species distribution and compositional dissimilarity. 
We evaluated the relative importance of contemporary and historical processes on patterns of dissimilarity in amphibian total species and endemic species composition in the Cerrado using the following framework. First, we compared whether and how environmental gradients within the Cerrado are correlated with dissimilarity in anuran endemic vs. total species composition. Then, we expanded the geographical extent of our analysis to neighboring eco-regions, where sister-taxa to Cerrado amphibians are distributed. Our ultimate goal was to test if we could detect an imprint of historical constraints through environmental niche conservatism on beta diversity. We predicted that (i) dissimilarities in assemblage composition among localities within the Cerrado would correlate with changes in climate and topography across the Cerrado (the local effect), (ii) the degree of environmental overlap between areas within the Cerrado and adjoining eco-regions would further influence species distribution and compositional dissimilarity (the regional effect), and (iii) this regional influence would be particularly strong for endemic, narrowly distributed Cerrado species, since most species belong to lineages originated in adjoining domains, especially in the Atlantic Forest.

\section{Methods}

\section{Study Area:}

The Cerrado phytogeographical domain is a mosaic of savannas, grasslands, woodlands and gallery forests that covers the Brazilian central plateau (Eiten 1972). Climate is highly seasonal, and mean temperatures range from 22 to $27^{\circ} \mathrm{C}$. For the purposes of domain delimitation, we followed Brazil's official vegetation classification (Veloso et al. 1991). We used the digitized map of terrestrial ecoregions of the world (Olson et al. 2001), and selected the Savanna ecoregion in Central Brazil, the Alto Paraná Atlantic Forest patches within the Savanna boundaries, and patches of Mato Grosso Seasonal Forests on the east bank of Araguaia River. To account for the wide and patchy transition zones between Cerrado and neighbor domains, we then included a $50 \mathrm{~km}$ buffer around the aforementioned region to arrive at a final study domain. 


\section{Species Data:}

We examined 31,024 specimens deposited in 11 Brazilian zoological collections (see list of visited collection in Appendix 1), and performed additional fieldwork in 11 Cerrado localities in some of the main survey gaps. Species identities were assessed through comparison with type specimens, species descriptions, and taxonomic revisions. When coordinates were not available in museum databases, precise locations were obtained directly from collectors. To avoid bias due to uneven microhabitat sampling across localities, and to enhance sample representativeness, we defined a site as the totality of sampled habitats within an $8.33 \times 8.33 \mathrm{~km}$ area. To avoid bias from omission errors, our analyses were restricted to sites from which at least 10 species were recorded. This data-cleaning process resulted in 64 wellsampled, precisely located sites within the study area (Figure 1A, appendix 2). We further generated a second dataset, containing only presence data for Cerrado endemic species. All localities that had no or only one endemic species were removed, reducing our data to 23 assemblages composed by two or more endemic species each (Figure 1B, appendix 2).

\section{Environmental data:}

We analyzed environmental correlates of beta diversity using two sets of environmental data that account for local and regional influences on species composition dissimilarity, respectively. The first set contained local climatic and topographic features expected to limit species distribution within the Cerrado due to species-specific physiological and microhabitat requirements. The second dataset comprised regional scale variables, which were based on the environmental conditions of the neighboring phytogeographical domains, and represent the historical component in our analysis. All raster and vector files were degraded to the resolution to 8.33 $\mathrm{km}$ to meet the resolution of species data. 

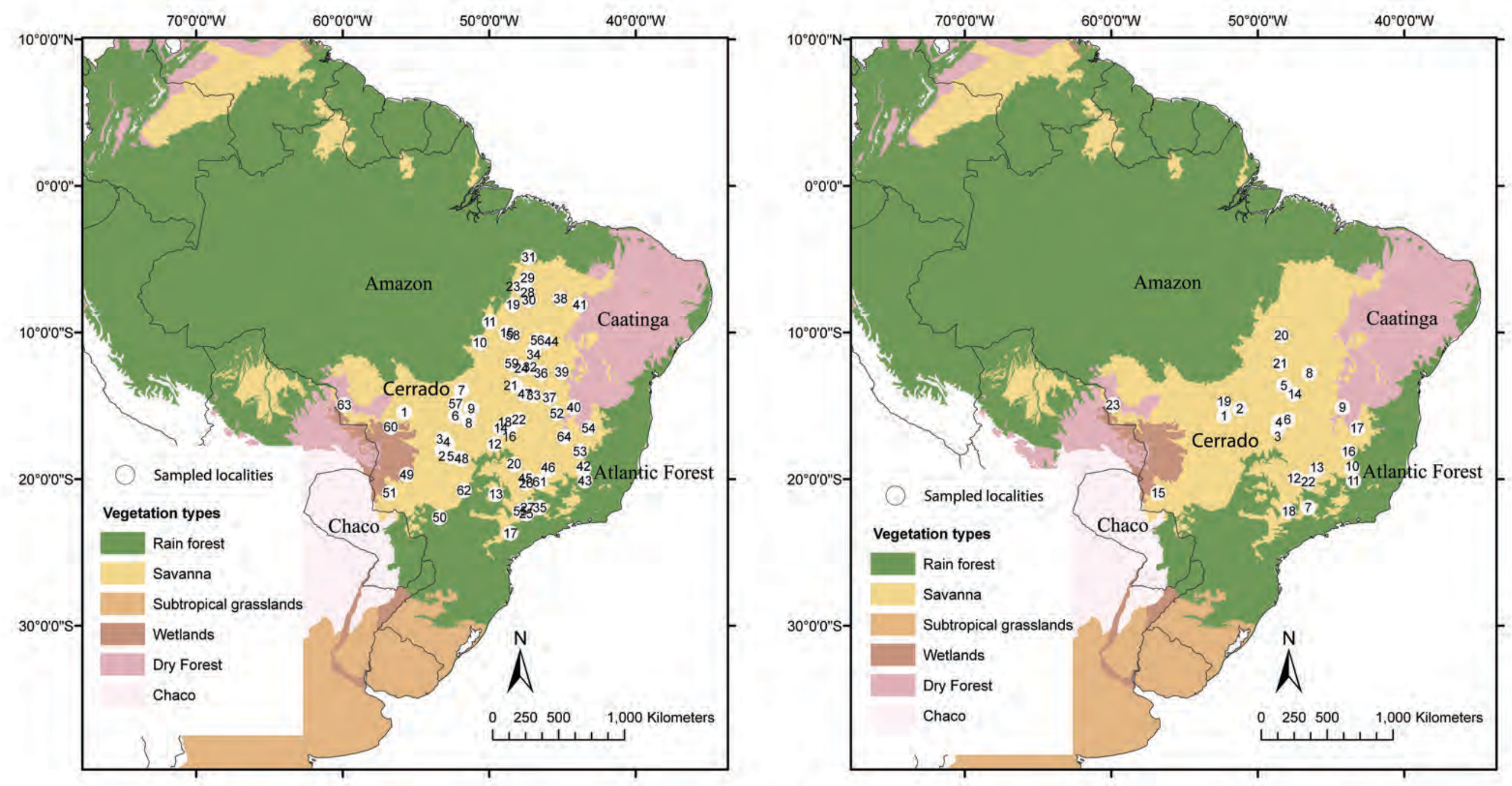

Figure 1. Maps depicting sampled sites. A - Location of the 64 sites analyzed in GDM1 and GDM2, with all species included. $B$ - Location of the 23 sites analyzed in GDM3 and GDM4, including only Cerrado endemics 
Table 1. Environmental predictors included in the GDM and the justification for inclusion of each predictor.

\begin{tabular}{|c|c|}
\hline Predictor & Justification for predictor \\
\hline \multicolumn{2}{|l|}{ Contemporary factors } \\
\hline Elevation & $\begin{array}{l}\text { Lower elevations have wider gallery forests, which } \\
\text { provide breeding habitat for forest species }\end{array}$ \\
\hline Slope & $\begin{array}{l}\text { Steeper slope areas have narrow temporary streams, } \\
\text { with a series of small pools and falls, which are } \\
\text { important breeding habitats for some species }\end{array}$ \\
\hline $\begin{array}{l}\text { Minimum temperature } \\
\text { of coldest month }\end{array}$ & $\begin{array}{l}\text { Low temperatures mainly affect the activity of } \\
\text { nocturnal species }\end{array}$ \\
\hline $\begin{array}{l}\text { Temperature annual } \\
\text { range }\end{array}$ & $\begin{array}{l}\text { A high temperature range affects the metabolism rate } \\
\text { of ectotherms throughout the year }\end{array}$ \\
\hline Annual precipitation & $\begin{array}{l}\text { Rainfall affects availability of sites for breeding and } \\
\text { tadpole development throughout the year }\end{array}$ \\
\hline $\begin{array}{l}\text { Precipitation of coldest } \\
\text { quarter }\end{array}$ & $\begin{array}{l}\text { Dry season rainfall affects availability of sites } \\
\text { especially for prolonged breeders and tadpoles with } \\
\text { slow developmental rates }\end{array}$ \\
\hline Soil moisture & $\begin{array}{l}\text { More saturated soils have a higher tendency to fill } \\
\text { and longer persistence of ponds }\end{array}$ \\
\hline
\end{tabular}

Historical factors

Environmental overlap More similar environmental conditions between the with each adjoining Cerrado and adjoining domains limit the geographic domain distribution of lineages that originated in these domains

\section{i. Contemporary Factors}

We selected biologically meaningful predictor variables related to climate, topography or soil composition, which could potentially explain amphibian distribution in the Cerrado (Table 1). A set of moderately highresolution bioclimatic variables was downloaded from WorldClim (Hijmans et 
al. 2005; available from http://www.worldclim.org/). These variables are spatially explicit estimates of annual means, seasonal extremes, and seasonality in temperature and precipitation, based on 50-year climate data (1950-2000). We discarded highly correlated variables ( $r>75 \%)$, and variables that would lead to similar interpretations, and used those presenting the highest range in the study region. The climate variables were reduced to minimum temperature of coldest month, temperature annual range, annual precipitation, and precipitation of coldest quarter. We obtained topography variables, namely elevation and slope, from EROS-USGS (U.S. Geological Survey Center for Earth Resources Observation and Science; http://eros.usgs.gov/products/elevation/hydro1k.html). Finally, we obtained soil moisture data from the Atlas of the Biosfere (Willmott \& Matsuura 2001; available from http://www.sage. wisc.edu/atlas/maps.php?catnum=3\&type=Ecosystems).

\section{ii. Historical Factors}

To test the impact of historical factors on beta diversity, we produced six new environmental variables, each representing the amount of climatic similarity between the Cerrado and one adjoining phytogeographical domain, where sister-taxa to Cerrado species are known to occur. First, we drew a $200 \mathrm{Km}$ buffer around the Cerrado and overlaped this zone with the map of terrestrial eco-regions (Olson et al. 2001). This resulted in six areas, representing the neighboring area in each adjoining domain: Amazonia, Atlantic Forest (only forest vegetation), Atlantic altitudinal grasslands, Caatinga, Pantanal, and Chaco. The Atlantic domain was split in two due to significant differences observed in the flora and fauna of forested areas vs. open, mountaintop landscapes. Second, to obtain a measure of environmental similarity between the Cerrado and each adjoining eco-region, we selected 200 random points within each of the delimited area in adjoining eco-regions and modeled the environmental space of this region with MAXENT (Phillips et al. 2006). The resulting projections of probability values over the Cerrado were used as new variables, representing the degree of similarity between every pixel within the Cerrado and each of the six adjoining domains. We used the same topography and soil data included in the GDM as 
well as additional climatic variables downloaded from WorldClim, since MAXENT obtains the best distribution model by letting the algorithm fit the distribution of maximum entropy to the data. Variables not weighted heavily in the model were not used. The following climatic variables were included: annual precipitation, isothermality, maximum temperature of warmest month, mean diurnal range, mean temperature of warmest quarter, mean temperature of wettest quarter, minimum temperature of coldest month, precipitation of coldest quarter, precipitation of driest month, precipitation of warmest quarter, precipitation of wettest month, precipitation seasonality, temperature annual range, and temperature seasonality.

\section{$\underline{\text { Quantification of beta diversity }}$}

To investigate environmental correlates of species dissimilarity (i.e., beta diversity), we developed a generalized dissimilarity model (GDM) using a downloadable package for R environment (http://www.biomaps.net.au/gdm/). The GDM package is a matrix-regression tool that models compositional dissimilarity as a function of environmental dissimilarity and geographic distance (Ferrier et al. 2007). GDM is a reformulation of the Mantel approach as a generalized linear model, in which a single response matrix can be modeled as a function of distance matrices of a number of explanatory variables (Manly 1986; Ferrier et al. 2007). Composition dissimilarity between each pair of sampled sites is calculated using the Bray-Curtis index. Then, for each environmental variable GDM derives a series of I-spline basis functions, and calculates the difference between each pair of sites. The same procedure is applied to geographic distance between pairs of sites. Finally GDM uses a maximum likelihood estimator to fit the coefficients to the I-spline basis functions, with composition dissimilarity as the response variable and the series of differences in the I-spline functions as predictors (Ferrier et al. 2007). Monte-Carlo permutations are used to assess the extent to which each predictor variable explains the observed response variation (Ferrier et al. 2004). Environmental variables that fail to significantly affect the dissimilarity in species composition are removed from the model. Further, the function describing the association between compositional dissimilarity and 
environmental dissimilarity is used to predict compositional dissimilarity across the area from which the assemblages were sampled.

Using GDM to investigate contemporary and historical drivers of beta diversity To evaluate the importance of contemporary and historical predictors of beta diversity, we produced and compared four generalized dissimilarity models: (i) GDM1 was based on an environmental dataset exclusively composed of contemporary environmental predictors, and composition data from total amphibian species in 64 sampled assemblages; (ii) GDM2 described the combined effects of historical and contemporary factors on overall diversity patterns, using this same total species sample; (iii) GDM3 described the effects of contemporary factors on Cerrado endemics only; and (iv) GDM4 described the combined effects of historical and contemporary factors on endemics only.

From each GDM we produced a map that shows predicted differences in community composition for any two locations, based on their environmental conditions. This procedure allows the classification of any region into a predefined number of environmental landscapes, through multidimensional scaling techniques (Ferrier et al. 2007). Because the Cerrado contacts six main South American phytogeographical domains, we arbitrarily set the number of landscapes to six.

\section{Results}

Compositional dissimilarity across Cerrado amphibian assemblages is partially explained by the contemporary environmental conditions. A GDM using contemporary predictors and total species assemblage (GDM1) identified six out of seven environmental variables as significantly correlated with species dissimilarity, and explained $42.35 \%$ of the variance in compositional dissimilarity. The inclusion of geographical distance increased the variance explained to $44.40 \%$. Minimum temperature of coldest month, elevation, annual precipitation, and precipitation of the coldest quarter were the most important variables contributing to the model (Table 2). The inclusion of historical predictors in the GDM of the total species assemblage (GDM2) slightly improved prediction of beta diversity to $43.83 \%$. The 
inclusion of geographic distance increased the variance explained to $45.05 \%$. While historical variables were selected in this model, the same variables as identified in GDM1 explained most of the variation (Table 2).

Model performance was considerably better when Cerrado endemics were analyzed. GDM3, developed for endemic species based on contemporary factors, selected all seven environmental variables, explained $52.62 \%$ of the variance in compositional dissimilarity, and explained variance only slightly increased (52.84\%) with the inclusion of geographical distance. The most important variables contributing to the model were soil moisture, precipitation of the coldest quarter, elevation, and slope (Table 2). GDM4, developed for endemic species based on historical and contemporary factors, selected nine out of thirteen environmental variables, explained $55.30 \%$ of the variance in compositional dissimilarity, and explained variance only slightly increased $(55.32 \%)$ with the inclusion of geographical distance. The most important variables contributing to the model were environmental overlap with Pantanal, Caatinga and Atlantic Forest, as well as slope, elevation and soil moisture (Table 2). Two variables, minimum temperature of the coldest month and precipitation of the coldest quarter were chosen in GDM3 but not in GDM4. These variables were somewhat correlated with the regional variables (absolute values of $r$ range from 0.05 to 0.73 ), however, regional variables with the highest correlation to local variables had relatively low partial contribution to the model.

Table 2. Environmental predictors used to produce the generalized dissimilarity models. Values indicate the y-axis range for each partial response curve. Higher ranges indicate greater influence on the predicted beta diversity.

\begin{tabular}{lcccc}
\hline & \multicolumn{4}{c}{ Partial contribution } \\
\hline Predictor & GDM1 & GDM2 & GDM3 & GDM4 \\
\hline Elevation & 0.55 & 0.52 & 1.86 & 1.73 \\
Slope & 0.12 & 0.10 & 1.79 & 1.87 \\
Minimum temperature of & 0.76 & 0.70 & 0.24 & $\mathrm{n} / \mathrm{s}$ \\
coldest month & & & &
\end{tabular}




$\begin{array}{lcccc}\text { Temperature annual range } & \mathrm{n} / \mathrm{s} & 0.03 & 0.60 & 1.31 \\ \text { Annual precipitation } & 0.30 & 0.22 & 0.01 & \mathrm{n} / \mathrm{s} \\ \text { Precipitation of coldest } & 0.28 & 0.23 & 2.02 & \mathrm{n} / \mathrm{s} \\ \text { quarter } & & & & \\ \text { Soil moisture } & 0.09 & 0.08 & 3.51 & 1.99 \\ \text { Pantanal } & - & 0.17 & - & 2.38 \\ \text { Chaco } & - & 0.04 & - & \mathrm{n} / \mathrm{s} \\ \text { Amazonia } & - & 0.02 & - & 0.58 \\ \text { Atlantic Forest } & - & 0.07 & - & 1.42 \\ \text { Caatinga } & - & 0.15 & - & 2.10 \\ \text { Campos } & - & 0.08 & - & 0.58\end{array}$
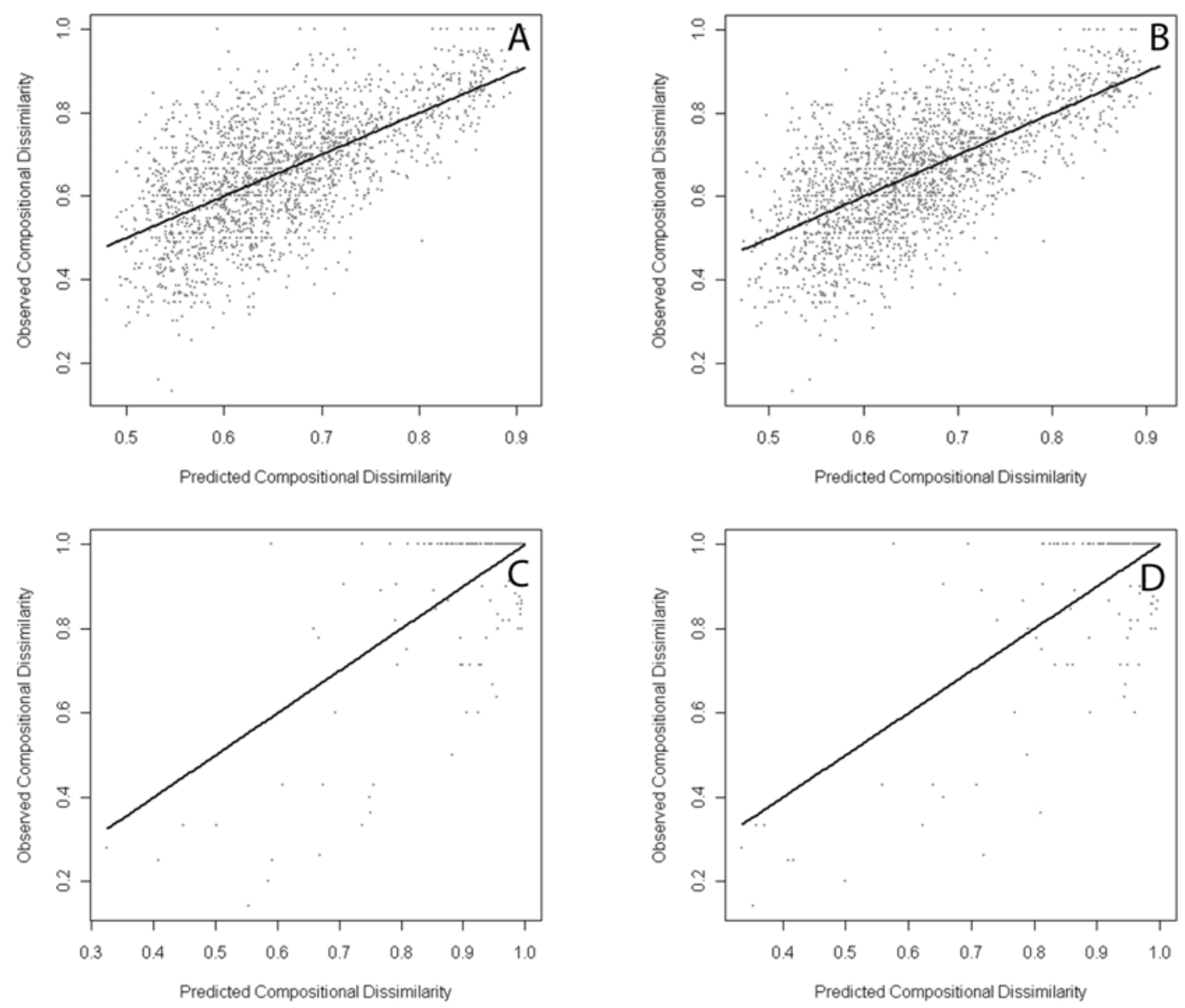

Figure 2. Validation of the GDM models. The observed response is the BrayCurtis dissimilarity. A - GDM1: all species and local predictors; B - GDM 2 - all species and local + regional predictors; C - GDM3 - endemic species and local predictors; D - endemic species and local + regional predictors. 
The models produced from the four different combinations of predictor and response datasets resulted in four different predictive maps subdividing the study area into six landscapes. Landscapes resulting from GDM1 and GDM2, both based on total species assemblage, showed similar patterns, while the landscapes from GDM3 or GDM4, based on endemics, were more distinct from each other. Further, landscapes were more continuous in the maps resulting from GDM1 and GDM2, whereas GDM3 and GDM4 had more scattered and disjunct landscapes. Both GDM1 and GDM2 identified the same three landscapes: the transition between Cerrado and Caatinga, western Cerrado, and central Cerrado. However, the northernmost landscape of GDM1 is divided in two landscapes in GDM2, whereas the southeastern Cerrado consists of two landscapes in GDM1 and one landscape in GDM2. Although elevation had a high partial contribution in all four models, it clearly influences landscape subdivision for endemic species models (GDM3 and GDM4), but this not so evident in the landscape maps for total species models. Area 4 in GDM 3 represents the mountain ranges, and 'orange' represents lowlands. Similarly, in GMD 4 area 3 represents lowlands, whereas there is no distinction between mountain ranges and plateaus, all are represented by area 4 . 

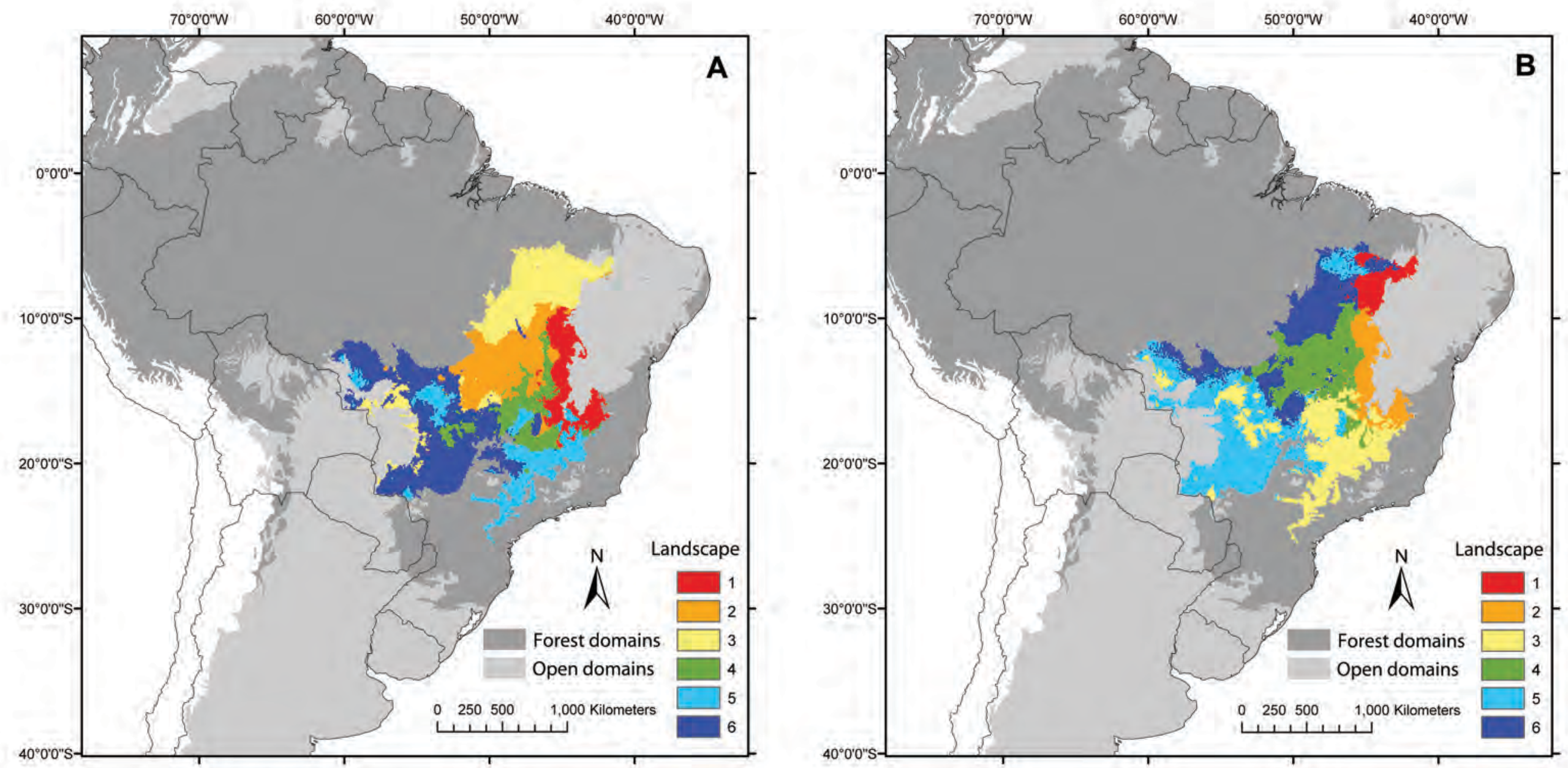

Figure 3. Maps depicting expected anuran assemblages compositional dissimilarity in the Cerrado that resulted from two of different combinations of environmental predictors and anuran assemblages. A - Total assemblage dissimilarity predicted by local scale variables (GDM1); B - total assemblage dissimilarity predicted by local and regional scale environmental variables (GDM2) 

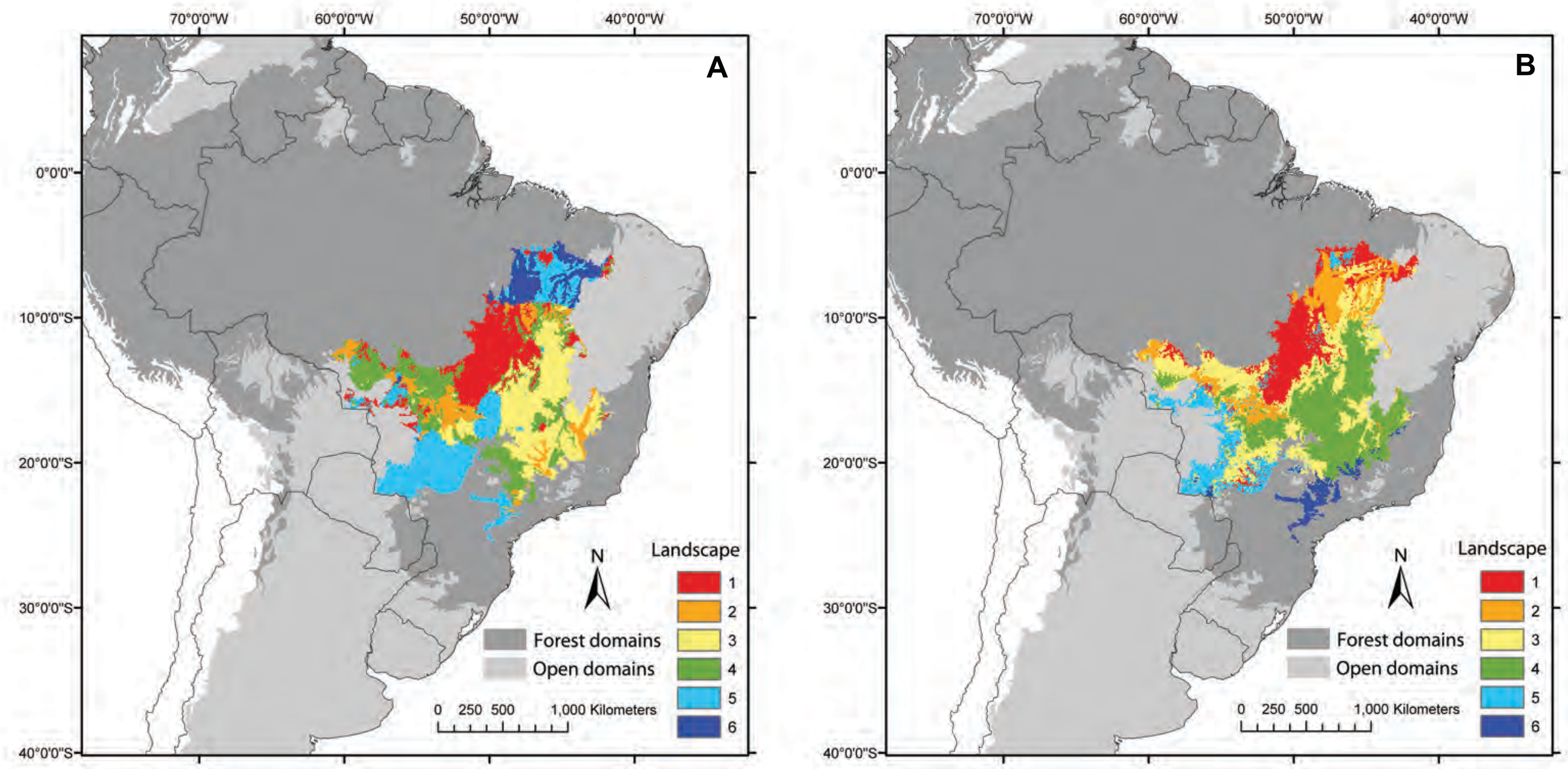

Figure 4. Maps depicting expected composition of endemic anurans compositional dissimilarity in the Cerrado that resulted from two of the different combinations of environmental predictors and anuran assemblages. A - Endemic species dissimilarity predicted by local scale variables (GDM3); B - endemic species dissimilarity predicted by local and regional scale environmental variables (GDM4) 


\section{Discussion}

Patterns of beta diversity in the Brazilian Cerrado appear to be strongly influenced by the environmental gradients in the region. Half of the dissimilarity in total species and endemic species assemblages can be explained by contemporary environmental factors, relating to anuran ecophysiological constraints. In addition, apart from the actual environmental gradients within the Cerrado, three other factors seem to influence patterns of beta diversity: 1) the similarity of environmental conditions across some landscapes to adjoining ecoregion; 2) the relationship of Cerrado endemics to lineages that likely originated in neighbor biomes, especially Atlantic Forest; and 3 ) the tendency of species to retain their ancestor features, resulting in the conservatism of climatic niches (Felsenstein 1985; Brooks \& McLennan 1991). In particular, we found that endemic species may be responding to environmental conditions based on how similar they are to neighbor biomes, since their sister-species are mostly distributed in these regions (Faivovich et al. 2005; Hedges et al. 2008; Padial et al. 2008). More broadly, factors acting across different spatial and temporal scale seem to interact to generate a complex beta-diversity pattern. Our results are consistent with increasing evidence that historical factors can have an important influence on current patterns of biodiversity (Cadle \& Greene 1993; Wiens \& Donoghue 2004; Graham et al. 2006; Ricklefs 2006; Carnaval et al. 2009).

Contemporary predictors, principally minimum temperature of the coldest month, elevation, annual precipitation, and precipitation of the coldest quarter, explained beta diversity for total species assemblages. These variables were chosen by the GDM regardless of the inclusion of the historical variables; the later explained very little variation in the model when they were included. The environmental variables that were chosen are related to physiological constraints and the availability of breeding habitats for different reproductive modes. Minimum temperature of the coldest month, the most important selected factor, may limit the geographic distribution of species, since the activity of nocturnal ectotherms can be strongly influenced by the lower temperatures faced by individuals (Hillman et al. 2009). The second most important variable in the GDM was elevation, which is considered a major determinant of the diversity of species and reproductive modes in 
Atlantic forest anurans (Haddad \& Prado 2005). In the Cerrado, dense riverine forests cover warmer lowlands on the northern and western parts of the biome, temporary streams are present in highlands and mountains in southeastern, while plateaus offer a lower range of habitat types. This microhabitat turnover caused by the altitudinal gradient may also favor beta diversity. Finally, annual precipitation and precipitation of the coldest quarter directly relate to breeding habitat availability for the great majority of Cerrado anuran species, which breed in temporary water bodies (Brasileiro et al. 2005; Brasileiro et al. 2008; Araujo et al. 2009; Valdujo et al. 2009). Species breeding sites vary in terms of ponds size and hydroperiod. Small rain puddles may be sufficient for some fast developing species, such as Dendropsophus rubicundulus (Barreto \& Moreira 1996), while other species, such as Pseudis spp. which may take more than a year to complete tadpole development (Fabrezi et al. 2009), need larger permanent or semi-permanent ponds, and may require a greater amount of rain to breed.

When the resulting GDMs based on complete assemblages were mapped, we find that regions roughly correspond to subdivisions of the Cerrado based on woody-plants (Ratter et al. 2003), anuran lineages (Chapter 1 / 2), and weakly, on vegetation physiognomy (Silva et al. 2006). Maps based on total assemblage dissimilarity were similar; therefore we only describe the results with the complete set of variables. There is a fair correspondence between the configuration of the six landscapes obtained by the GDM with the subdivision into provinces based on woody-plants dissimilarity (Ratter et al. 2003): landscape 3 corresponds to Southern and to Central and Southeastern woody-plant provinces, 1 and 2 subdivides the North and Northeastern woody-plant provinces, and finally landscapes 4, 5, and 6 subdivides the Central-Western woody-plant province. Further, the subdivisions that we obtained are consistent with the distribution of anuran lineages in the Cerrado and neighboring ecoregions. For instance, species related to Atlantic lineages are distributed in landscape 3, species shared with Caatinga are distributed in landscape 2 and 1, species related to Amazonian lineages are distributed in landscape 6 and partially in 4, and finally, Chaco and Pantanal species occur in landscape 5. However, the correspondence with the ecological units presented by (Silva et al. 2006), is less evident. Silva 
et al. (2006) proposed a hierarchical classification of the environmental diversity of the Cerrado, with ecological units nested within landscapes. Landscapes were based on landform and vegetation types, and ecological units were based on physiognomy, phenology, topography, and drainage. Our landscapes 3,2 , and 1 overlap to some extent with ecological units 1A, 1D, and 3D, respectively. However, landscapes 4, 5, and 6 partially overlap with several ecological units. The correspondence of anurans and woody-plants species maps, and the contrast between them and the vegetation physiognomy map suggests a combined role of both current environmental factors and historical contingency on assemblage composition. Different species may occur in similar physiognomies across the Cerrado suggesting that historical factors may limit species distributions (Ricklefs \& Schluter 1993).

The GDM based on endemic species selected different variables compared to the GDM on total assemblages, and were mostly related to availability of breeding habitats for different reproductive modes.

Contemporary predictors, principally soil moisture, elevation, slope, and precipitation of the coldest quarter explained assemblage dissimilarity for endemic species. The only variable common between the total and the endemic models was elevation, highlighting the uniqueness of high elevation anuran assemblages (e.g. Eterovick \& Sazima 2000; Leite et al. 2008). More steep relief, represented by higher slope, combines with elevation and precipitation for the availability of temporary mountain streams, allowing the occurrence stream-breeder species (Wells 2007). Soil moisture relates to habitat availability specifically for pond-breeder species, especially those that rely on semi-permanent ponds. These ponds are more common when soil is saturated. Since the coldest season in the Cerrado is also the dry season, precipitation of the coldest quarter may affect the availability of breeding sites for species that breed in the winter on temporary water bodies.

When we included regional-scale variables, overlap with the Pantanal, Caatinga, and Atlantic Forest also entered the model, suggesting a greater importance of historical contingencies on species that originated in the Cerrado. This association may be due to the fact that many endemic species are representatives of taxa diversified, and probably originated, in one of the 
neighbor domains, especially Atlantic Forest (e.g. those in genera Bokermannohyla, Crossodactylus, Hylodes, and Phasmahyla; see range maps in IUCN 2010). The fact that species share some morphological, behavioral and ecophysiological characteristics with their closest relatives (Felsenstein 1985), may limit their movement into regions with novel environments (Ricklefs 2006). These results indicate that environmental factors combine with differences in regional species pools, determined by the long-term history of speciation and extinctions, and contribute to beta-diversity patterns by restricting species belonging to some lineages to a specific part of the environmental gradient.

Maps depicting the results of GDMs for endemic species differed from each other and landscapes have weaker correspondence with other published Cerrado subdivisions. The map resulting from GDM 3 shows a strong influence of elevation and slope, and subdivides the Cerrado according to the topography. Landscape 3 corresponds to plateau areas, landscape 2 represents the highlands and landscape 4 represents lowlands. The map resulting from GDM4 shows all lowlands combined in landscape 3, while plateaus and highlands are in landscape 4. GDM4 landscapes have a remarkable influence of the environmental similarity with adjoining domains: landscape 6 overlaps with Atlantic Forest environmental envelope, landscape 5 overlaps with Chaco environmental envelope and landscape 1 overlaps with Amazon environmental envelope. Maps depicting landscape subdivision based on endemic species reinforce the importance of historical factors combined with environmental heterogeneity in influencing beta diversity in the Cerrado. Apparently this pattern was obscured by widespread species when we included complete assemblages. Although regional scale environmental variables were selected, they were not the most important variables contributing to the model when analyzing the complete communities.

The association of environmental gradients and compositional dissimilarity in Cerrado anurans had never been assessed directly prior to this study. However, it was not unexpected due to the known relationship between amphibian species richness and environmental variables described in the Brazilian Cerrado (Diniz-Filho et al. 2008). Anuran richness tends to be greater in the southern Cerrado with colder areas having greater numbers of 
species (Diniz-Filho et al. 2008), though Diniz-Filho et al. (2005; 2008) cautioned that this result was preliminary given sparse sampling in the north. In our study, the temperature of the coldest month was also an important variable. Additionally, temperature related variables, elevation and the environmental overlap with Atlantic Forest also contributed to amphibian dissimilarity. Since we included only well sampled sites in our analysis and corrected for sample size, this pattern should not be biased by sampling effort. Further, the greater physiognomical heterogeneity in the southern Cerrado (Silva et al. 2006) may enhance both beta-diversity and broad scale alpha diversity (10.000 km²; Diniz-Filho et al. 2008). In contrast to our results and to Diniz-Filho et al. (2008), Vasconcelos et al. (2010) reported a weak association between environmental factors and species richness in anuran communities in Brazil, but most of their assemblages were in Atlantic Forest and few data came from the Cerrado, Caatinga, Pantanal and Amazonia. In spite of the weak relationship, they described total rainfall and rainfall seasonality as the most important variables in the model. Temperature and elevation were negatively correlated to each other and explained very little of the richness distribution. The difference between Diniz-Filho et al. (2008) and our results to those of Vasconcelos et al. (2010) may be caused by the geographic extent sampled in each study; the former sampled across environmental gradients in the Cerrado while the later sampled across Brazil but with most localities in the Atlantic Forest.

Here we propose a method to include historical factors in beta diversity, which may also be useful for other spatially explicit analysis. We demonstrate that history may shape communities and beta diversity is likely generated by a combined effect of environmental filtering and lineage origin (Graham \& Fine 2008; Vamosi et al. 2009). The history of lineages may restrict species distributions to part of the environmental gradient in a region; therefore in regions where species belong to lineages that have different biogeographical histories we expect an increases beta diversity. Further, heterogeneity in habitat and topography influences the environmental conditions and the availability of resources, as well as speciation rates (Rahbek \& Graves 2001; Jetz \& Rahbek 2002). These processes seem to result in a historical imprint not only on recently dispersed species, but also on 
endemic species that evolved in situ and conserved some of their ancestors' environmental requirements. This interplay of environmental and historical constraints on anuran richness patterns was demonstrated by Buckley \& Jetz (2007) in global scale, and our results reinforce the importance of taking biogeographical history into account when analyzing spatial patterns of species diversity at a regional scale. Future phylogenetic and phylogeographic studies including Cerrado species and their sister-taxa in neighbor domains will provide additional tests of the importance of historical and contemporary factors on patterns of biodiversity in this global hotspot.

\section{References}

Araujo, C. O., T. H. Condez \& R. J. Sawaya (2009). Anfíbios Anuros do Parque Estadual das Furnas do Bom Jesus, sudeste do Brasil, e suas relações com outras taxocenoses no Brasil. Biota Neotropica 9: 77-98.

Ashcroft, M. B., J. R. Gollan, D. P. Faith, G. A. Carter, S. A. Lassau, S. G. Ginn, M. W. Bulbert \& G. Cassis (2010). Using generalised dissimilarity models and many small samples to improve the efficiency of regional and landscape scale invertebrate sampling. Ecological Informatics 5: 124-132.

Azeria, E. T., D. Fortin, C. Hebert, P. Peres-Neto, D. Pothier \& J.-C. Ruel (2009). Using null model analysis of species co-occurrences to deconstruct biodiversity patterns and select indicator species. Diversity and Distributions 15: 958-971.

Barreto, L. \& G. Moreira (1996). Seasonal variation in age structure and spatial distribution of a savanna larval anuran assemblage in central Brazil. Journal of Herpetology 30: 87-92.

Brasileiro, C. A., R. J. Sawaya, M. C. Kiefer \& M. Martins (2005). Amphibians of an open Cerrado fragment in southeastern Brazil. Biota Neotropica 5: 93-109.

Brasileiro, C. A., E. M. Lucas, H. M. Oyamaguchi, M. T. C. Thomé \& M. Dixo (2008). Anurans, northern Tocantins River Basin, states of Tocantins and Maranhão, Brazil. Check List 4: 185-197.

Brooks, D. \& D. A. McLennan (1991). Phylogeny, Ecology and Behavior - A Research Program in Comparative Biology. Chicago, The University of Chicago Press.

Buckley, L. B. \& W. Jetz (2007). Environmental and historical constraints on global patterns of amphibian richness. Proceedings of the Royal Society B: Biological Sciences 274: 1167-1173.

Cadle, J. E. \& H. W. Greene (1993). Phylogenetic patterns, biogeography, and the ecological structure of neotropical snake assemblages.

Species diversity in ecological communities, historical and geographical perspectives. R. E. Ricklefs and D. Schluter. Chicago, The University of Chicago Press: 281-293. 
Carnaval, A. C., M. J. Hickerson, C. F. B. Haddad, M. T. Rodrigues \& C. Moritz (2009). Stability predicts genetic diversity in the Brazilian Atlantic forest hotspot. Science 323: 785-789.

Costa, L. P. (2003). The historical bridge between the Amazon and the Atlantic Forest of Brazil: a study of molecular phylogeography with small mammals. Journal of Biogeography 30: 71-86.

Diniz-Filho, J. A. F., L. M. Bini, C. M. Vieira, D. Blamires, L. Terribile, R. Bastos, G. de Oliveira \& B. Barreto (2008). Spatial patterns of terrestrial vertebrate species richness in the Brazilian Cerrado. Zoological Studies 47: 146-157.

Diniz-Filho, J. A. F., R. P. Bastos, T. F. L. V. B. Rangel, L. M. Bini, P. Carvalho \& R. J. Silva (2005). Macroecological correlates and spatial patterns of anuran description dates in the Brazilian Cerrado. Global Ecology and Biogeography 14: 469-477.

Eiten, G. (1972). The Cerrado vegetation of Brazil. Botanical Review 38: 201341.

Ellwood, M. D. F., A. Manica \& W. A. Foster (2009). Stochastic and deterministic processes jointly structure tropical arthropod communities. Ecology Letters 12: 277-284.

Eterovick, P. C. \& I. Sazima (2000). Structure of an anuran community in a montane meadow in southeastern Brazil: effects of seasonality, habitat, and predation. Amphibia-Reptilia 21: 439-461.

Eterovick, P. C. \& G. W. Fernandes (2002). Why do breeding frogs colonize some puddles more than others? Phyllomedusa 1: 31-40.

Fabrezi, M., S. I. Quinzio \& J. Goldberg (2009). Giant tadpole and delayed metamorphosis of Pseudis platensis Gallardo, 1961 (Anura, Hylidae). Journal of Herpetology 43: 228-243.

Faivovich, J., C. F. B. Haddad, P. C. A. Garcia, D. R. Frost, J. A. Campbell \& W. C. Wheeler (2005). Systematic review of the frog family Hylidae, with special reference to Hylinae: phylogenetic analysis and taxonomic revision. Bulletin of the American Museum of Natural History 294: 1240.

Felsenstein, J. (1985). Phylogenies and the comparative method. American Naturalist 125: 1-15.

Ferrier, S. (2002). Mapping spatial pattern in biodiversity for regional conservation planning: where to from here? Systematic Biology 51: 331-363.

Ferrier, S., G. V. N. Powell, K. S. Richardson, G. Manion, J. M. Overton, T. F. Allnutt, S. E. Cameron, K. Mantle, N. D. Burgess, D. P. Faith, J. F. Lamoreux, G. Kier, R. J. Hijmans, V. Funk, G. A. Cassis, B. L. Fisher, P. Flemons, L. D., J. C. Lovett \& R. S. A. R. Van-Rompaey (2004). Mapping more of terrestrial biodiversity for global conservation assessment. BioScience 54: 1101-1109.

Ferrier, S., G. Manion, J. Elith \& K. Richardson (2007). Using generalized dissimilarity modelling to analyse and predict patterns of beta diversity in regional biodiversity assessment. Diversity and Distributions 13: 252264.

Graham, C. H., C. Moritz \& S. E. Williams (2006). Habitat history improves prediction of biodiversity in rainforest fauna. Proceedings of the National Academy of Sciences 103: 632-636. 
Graham, C. H. \& P. V. A. Fine (2008). Phylogenetic beta diversity: linking ecological and evolutionary processes across space in time. Ecology Letters. 11: 1265-1277.

Haddad, C. F. B. \& C. P. A. Prado (2005). Reproductive modes in frogs and their unexpected diversity in the Atlantic Forest of Brazil. BioScience 55: 207-217.

Hedges, S. B., W. E. Duellman \& M. P. Heinicke (2008). New World directdeveloping frogs (Anura : Terrarana): Molecular phylogeny, classification, biogeography, and conservation. Zootaxa: 1-182.

Hijmans, R. J., S. E. Cameron, J. L. Parra, P. G. Jones \& A. Jarvis (2005). Very high resolution interpolated climate surfaces for global land areas. International Journal of Climatology 25: 1965-1978.

Hillman, S. S., P. C. Withers, R. C. Drewes \& S. D. Hillyard (2009). Ecological and Environmental Physiology of Amphibians. New York, Oxford University Press Inc.

IUCN (2010). IUCN Red List of Threatened Species. Version 2010.4. $<$ http://www.iucnredlist.org >. Retrieved 3 January 2011, 2011.

Jankowski, J. E., A. L. Ciecka, N. Y. Mayer \& K. N. Rabenold (2009). Beta diversity along environmental gradients: implications of habitat specialization in tropical montane landscapes. Journal of Animal Ecology 78: 315-327.

Jetz, W. \& C. Rahbek (2002). Geographic Range Size and Determinants of Avian Species Richness. Science 297: 1548-1551.

Leite, F. S. F., F. A. Juncá \& P. C. Eterovick (2008). Status do conhecimento, endemismo e conservação de anfíbios anuros da Cadeia do Espinhaço, Brasil. Megadiversidade 4: 182-200.

Manly, B. F. J. (1986). Randomization and regression methods for testing for associations with geographical, environmental and biological distances between populations. Researches on Population Ecology 28: 201-218.

Oliveira-Filho, A. T. \& J. A. Ratter (1995). A study of the origin of central Brazilian forests by the analysis of plant species distribution patterns. Edinburgh Journal of Botany 52: 141-194.

Olson, D. M., E. Dinerstein, E. D. Wikramanayake, N. D. Burgess, G. V. N. Powell, E. C. Underwood, J. A. D'Amico, I. Itoua, H. E. Strand, J. C. Morrison, C. J. Loucks, T. F. Allnut, T. H. Ricketts, Y. Kura, J. F. Lamoreux, W. W. Wettengel, P. Hedao \& K. R. Kassem (2001). Terrestrial ecoregions of the world: a new map of life on earth. BioScience 51: 933-938.

Overton, J. M., G. M. Barker \& R. Price (2009). Estimating and conserving patterns of invertebrate diversity: a test case of New Zealand land snails. Diversity and Distributions 15: 731-741.

Padial, J. M., J. C. Chaparro \& I. de-la-Riva (2008). Systematics of Oreobates and the Eleutherodactylus discoidalis species group (Amphibia, Anura), based on two mitochondrial DNA genes and external morphology. Zoological Journal of the Linnean Society 152: 737-773.

Phillips, S. J., R. P. Anderson \& R. E. Schapire (2006). Maximum entropy modeling of species geographic distributions. Ecological Modelling 190: 231-259. 
Qian, H. (2009). Global comparisons of beta diversity among mammals, birds, reptiles, and amphibians across spatial scales and taxonomic ranks. Journal of Systematics and Evolution 47: 509-514.

Rahbek, C. \& G. R. Graves (2001). Multiscale assessment of patterns of avian species richness. Proceedings of the National Academy of Sciences 98: 4534-4539.

Ratter, J. A., S. Bridgewater \& J. F. Ribeiro (2003). Analysis of the floristic composition of the Brazilian cerrado vegetation III: comparison of the woody vegetation of 376 areas. Edinburgh Journal of Botany 60: 57109.

Ricklefs, R. E. \& D. Schluter (1993). Species Diversity in Ecological Communities, Historical and Geographical Perspectives. Chicago, The University of Chicago Press.

Ricklefs, R. E. (2006). Evolutionary diversification and the origin of the diversity-environment relationship. Ecology 87: S3-S13.

Silva, J. F., M. R. Fariñas, J. M. Felfili \& C. A. Klink (2006). Spatial heterogeneity, land use and conservation in the cerrado region of Brazil. Journal of Biogeography 33: 536-548.

Silva, J. M. C. (1996). Distribution of Amazonian and Atlantic Forest Birds in Gallery Forests of the Cerrado Region, South America. Ornitologia Neotropical 7: 1-18.

Thomassen, H. A., W. Buermann, B. Milá, C. H. Graham, S. E. Cameron, C. J. Schneider, J. P. Pollinger, S. Saatchi, R. K. Wayne \& T. B. Smith (2010). Modeling environmentally associated morphological and genetic variation in a rainforest bird, and its application to conservation prioritization. Evolutionary Applications 3: 1-16.

Valdujo, P. H., R. S. Recoder, M. M. Vasconcellos \& A. S. Portella (2009). Amphibia, Anura, São Desidério, western Bahia uplands, northeastern Brazil. Check List 5: 903-911.

Vamosi, S. M., S. B. Heard, J. C. Vamosi \& C. O. Webb (2009). Emerging patterns in the comparative analysis of phylogenetic community structure. Molecular Ecology 18: 572-592.

Vasconcelos, T. S., T. G. Santos, D. C. Rossa-Feres \& C. F. B. Haddad (2009). Influence of the environmental heterogeneity of breeding ponds on anuran assemblages from southeastern Brazil. Canadian Journal of Zoology 87: 699-707.

Vasconcelos, T. S., T. G. Santos, C. F. B. Haddad \& D. C. Rossa-Feres (2010). Climatic variables and altitude as predictors of anuran species richness and number of reproductive modes in Brazil. Journal of Tropical Ecology 26: 423-432.

Veloso, H. P., A. L. R. Rangel-Filho \& J. C. Lima (1991). Classificação da vegetação brasileira, adaptada a um sistema universal. Rio de Janeiro, Fundação Instituto Brasileiro de Geografia e Estatística.

Wells, K. D. (2007). The Ecology and Behavior of Amphibians. Chicago and London, The University of Chicago Press.

Whittaker, R. H. (1960). Vegetation of the Siskiyou Mountains, Oregon and California. Ecological Monographs 30: 279-338.

Wiens, J. J. \& M. J. Donoghue (2004). Historical biogeography, ecology and species richness. Trends in Ecology \& Evolution 19: 639-644. 
Wiens, J. J. \& C. H. Graham (2005). Niche conservatism: integrating evolution, ecology, and conservation biology. Annual Review of Ecology Evolution and Systematics 36: 519-539.

Willmott, C. J. \& K. Matsuura (2001). Terrestrial water budget data archive: monthly time series (1950 - 1999). Available at http://www.sage. wisc.edu/atlas/maps.php?catnum=3\&type=Ecosystem s. 
Appendix 1. List of visited zoological collections: Museu de Zoologia da Universidade de São Paulo (MZUSP), Museu Nacional do Rio de Janeiro (MNRJ), Coleção Herpetológica da Universidade de Brasília (CHUNB), Coleção Herpetológica da Universidade Federal de Goiás (ZUFG), Museu Paraense Emílio Goeldi (MPEG), Coleção Zoológica da Universidade Federal do Mato Grosso (ZUFMT), Coleção Zoológica da Universidade Federal do Mato Grosso do Sul (ZUFMS), Museu de História Natural Adão Cardoso - Universidade Estadual de Campinas (ZUEC), Coleção Célio Haddad - Universidade Estadual Paulista, Campus Rio Claro (CFBH), Coleção Herpetológica do Museu de Ciências Naturais da PUC-Minas (MCN), Coleção do Departamento de Zoologia e Botânica (DZSJRP) housed at UNESP, São José do Rio Preto.

Appendix 2. List of localities included in GDM.

GDM1 and GDM2 (all species): 1. Chapada dos Guimaraes, MT (-15.4522, 55.8022); 2. Alto Taquari, MT (-18.0356, -53.2189); 3. Santa Rita do Araguaia, GO (17.2856, -53.1356); 4. Mineiros, GO (-17.6189, -52.8856); 5. Chapadão do Céu, GO (-18.4522, -52.6356); 6. Barra do Garças, MT (-15.7022, -52.3022); 7. Água Boa, MT (-13.9522, -51.8856); 8. Montes Claros de Goiás, GO (-16.0356 -51.3856); 9.

Britânia, GO (-15.2022, -51.2189); 10. Santa Terezinha, MT (-10.7022, -50.6356); 11. Caseara, TO (-9.2856, -49.9689); 12. Pontalina, GO (-17.6189, -49.6356); 13. Nova Itapirema, SP (-21.0356, -49.5522); 14. Goiânia, GO (-16.5356, -49.2189); 15. Paraíso do Tocantins, TO (-10.0356, -48.8022); 16. Silvânia, GO (-16.6189, 48.6356); 17. Buri, SP (-23.7022, -48.5522); 18. Alexânia, GO (-16.1189, -48.5522); 19. Palmeirante, TO (-8.1189, -48.3856); 20. Uberlândia, MG (-19.0356, -48.3022); 21. Minaçu, GO (-13.6189, -48.2189); 22. Brasília, DF (-15.9522, -47.9689); 23. Babaçulândia, TO (-6.8689, -47.8856); 24. Paranã, TO (-12.4522, -47.8022); 25. Rio Claro, SP (-22.3689 -47.4689); 26. Pedregulho, SP (-20.2856, -47.4689); 27. Pirassununga, SP (-21.9522, -47.3856); 28. Carolina, MA (-7.2856, -47.3856); 29. Porto Franco, MA (-6.2856, -47.3856); 30. Goiatins, TO (-7.7856, -47.3022); 31. Açailândia, MA (-4.8689, -47.3022); 32. Conceição do Tocantins, TO (-12.3689, 47.2189); 33. Flores de Goiás, GO (-14.2856, -46.9689); 34. Almas, TO (-11.2856, 46.9689); 35. Poços de Caldas, MG (-21.9522, -46.5522); 36. Combinado, TO (12.7856, -46.4689); 37. Jaborandi, BA (-14.4522, -45.8856); 38. Ribeiro Golçalves, PI (-7.7022, -45.1356); 39. São Desidério, BA (-12.7022, -45.0522); 40. Januária, MG (-15.1189, -44.2189); 41. Eliseu Martins, PI (-8.1189, -43.8022); 42. Jaboticatubas, MG (-19.2856, -43.5522); 43. Catas Altas, MG (-20.1189, -43.4689); 
44. Formosa do Rio Preto, BA (-10.6189, -46.1356); 45. Sacramento, MG (-19.9522, -47.3856); 46. São Gotardo, MG (-19.3689, -45.9689); 47. Alto Paraíso de Goiás (14.2022, -47.5522); 48. Aporé, GO (-18.6189, -51.8856); 49. Aquidauana, MS (19.7022, -55.6356); 50. Assis, SP (-22.6189, -53.3856); 51. Bonito, MS (-20.9522, 56.8022); 52. Chapada Gaúcha, MT (-15.5356, -45.3856); 53. Diamantina, MG (18.1189, -43.8022); 54. Grão Mogol, MG (-16.5356, -43.2189); 55. Itirapina, SP (22.2022, -47.8856); 56. Mateiros, TO (-10.5356, -46.7189); 57. Nova Xavantina, MT (-14.8689, -52.3022); 58. Palmas, TO (-10.2022, -48.3856); 59. Peixe, TO (-12.1189, -48.4689); 60. Poconé, MT (-16.4522, -56.7189); 61. São Roque de Minas, MG (20.2022, -46.5522); 62. Três Lagoas, MS (-20.7856, -51.7189); 63. Vila Bela da Santíssima Trindade, MT (-14.9522; -59.8856); 64. Buritizeiro, MG (-44.8771, 17.1189).

GDM3 and GDM 4 (endemic species): 1. Barra do Garcas, MT (-15.7022, -52.3022); 2. Britânia, GO (-15.2022, -51.2189); 3. Silvânia, GO (-16.6189, -48.6356); 4. Alexânia, GO (-16.1189, -48.5522); 5. Minaçu, GO (-13.6189, -48.2189); 6. Brasília (15.9522, -47.9689); 7. Poços de Caldas, MG (-21.9522, -46.5522); 8. Combinado, TO (-12.7856, -46.4689); 9. Januária, MG (-15.1189, -44.2189); 10. Jaboticatubas, MG (-19.2856, -43.5522); 11. Catas Altas, MG (-20.1189, -43.4689); 12.

Sacramento, MG (-19.9522, -47.3856); 13. Sao Gotardo, MG (-19.3689, -45.9689); 14. Alto Paraiso de Goias, GO (-14.2022, -47.5522); 15. Bonito, MS (-20.9522, 56.8022); 16. Diamantina, MG (-18.1189, -43.8022); 17. Grao Mogol, MG (-16.5356, -43.2189); 18. Itirapina, SP (-22.2022, -47.8856); 19. Nova Xavantina, MT (-14.8689, -52.3022); 20. Palmas, TO (-10.2022, -48.3856); 21. Peixe, TO (-12.1189, -48.4689); 22. São Roque de Minas, MG (-20.2022, -46.5522); 23. Vila Bela da Santíssima Trindade, MT (-14.9522, -59.8856). 
Discussão Geral e Conclusões 


\section{Discussão Geral e Conclusões}

Esta tese contribui para o entendimento dos fatores que influenciam a distribuição dos anfíbios anuros no Cerrado. A partir de uma ampla base de dados, descrevi os padrões de distribuição das espécies que ocorrem no Cerrado, dos gêneros primariamente associados aos biomas florestais que apresentam representantes no Cerrado e das mudanças na composição das comunidades. A partir dessas informações e de bases de dados ambientais de alta resolução, avaliei de que forma os fatores históricos, representados pela relação com biomas vizinhos, e os fatores ambientais, relacionados à topografia, solo e clima, determinam a distribuição dos táxons.

No primeiro capítulo atualizei a lista de espécies de anfíbios anuros do Cerrado para 204 espécies e descrevi os padrões de distribuição, avaliando endemismos, endemismos restritos (área de distribuição $<60.000 \mathrm{Km}^{2}$ ) e a forte estruturação espacial que se observa entre as espécies que ocorrem no Cerrado e em um domínio vizinho. Ao contrário do que se acreditava, $50 \%$ do total de espécies e mais de $70 \%$ das espécies típicas do Cerrado são endêmicas, e apenas $10 \%$ do total de espécies tem distribuição ampla, ocorrendo em mais de dois domínios na América do Sul. Dentre as endêmicas, destaca-se um conjunto de espécies pertencentes a diversos clados que apresenta distribuição bastante restrita, geralmente associada aos topos de montanha, no Maciço de Espinhaço, Serra da Canastra, Chapada dos Guimarães, e Chapada dos Veadeiros. As espécies que ocorrem no Cerrado e em mais um domínio são minoria e estão distribuídas pelas porções do Cerrado mais próximas do tal domínio vizinho. Apesar de não ser possível inferir os processos que deram origem a estes padrões a partir dos nossos dados, o fato de muitas dessas espécies pertencerem a linhagens inteiras de domínios florestais levanta a hipótese de que estas espécies possam ter dispersado dos domínios vizinhos para o Cerrado. Deste modo, proponho que a fauna do Cerrado possui uma identidade, representada pelas espécies endêmicas, mas com alguma influência dos domínios vizinhos, representados pelas espécies compartilhadas. Futuros estudos filogenéticos serão úteis para avaliar as relações históricas entre as espécies endêmicas 
do Cerrado e os domínios vizinhos, onde os demais representantes de suas linhagens ocorrem.

No segundo capítulo analisei a associação entre a distribuição dos gêneros de anfíbios anuros que ocorrem no Cerrado e as condições climáticas (representadas pela pluviosidade), características topográficas (representadas pela altitude média e variação altitudinal em volta de cada localidade) e a distância aos domínios florestais vizinhos (Amazônia e Mata Atlântica). Restringi-me aos 16 gêneros de distribuição predominantemente atlântica e amazônica que apresentam espécies no Cerrado, fossem elas endêmicas do domínio ou compartilhadas com outros domínios, para tentar recuperar a ligação histórica entre o Cerrado e os domínios florestais.

Os 16 gêneros analisados apresentam um padrão de distribuição do tipo "tabuleiro de xadrez" (Gotelli 2000), no qual algumas espécies nunca coocorrem. Contudo, esse padrão não aparece quando os gêneros são agrupados pelo domínio em que são mais frequentes (aqui chamado centro de diversidade), porque gêneros amazônicos tenderam a co-ocorrer entre si, o mesmo tendo ocorrido com os gêneros atlânticos. Contudo, os gêneros pertencentes a um grupo co-ocorreram com gêneros pertencentes ao outro grupo em poucas localidades, que podem ser consideradas zonas de contato entre os grupos. Dessa forma, foi possível acessar a importância da relação histórica com domínios vizinhos até mesmo para as espécies endêmicas do Cerrado, mas pertencentes a linhagens primariamente florestais. Tal padrão, geograficamente estruturado na distribuição e semelhante àquele observado para as espécies no primeiro capítulo, indica uma forma pela qual os fatores históricos operam na montagem das comunidades.

Ainda no segundo capítulo, demonstrei a relação dos fatores ambientais e históricos associados à ocorrência das espécies pertencentes a cada um dos grupos biogeográficos. Espécies pertencentes a gêneros atlânticos tendem a ocorrer em montanhas, em ambientes de baixa pluviosidade e próximas aos limites com a Mata Atlântica. Por outro lado, as espécies pertencentes a gêneros amazônicos tendem a ocorrer em vales próximos à Amazônia. De fato, mesmo nas áreas em que espécies de linhagens atlânticas e amazônicas co-ocorrem (e.g. Chapada dos Veadeiros), elas são encontradas em ambientes distintos: espécies de linhagens 
atlânticas tendem a ocorrer nos córregos, próximos à nascentes (e.g. Bokermannohyla pseudopseudis), enquanto as espécies de linhagens atlânticas tendem a ocorrer nas matas de galerias dos vales mais encaixados (e.g. Rhaebo guttatus). Esse padrão levanta a hipótese de que os nichos ambientais das espécies que ocorrem no Cerrado, mas pertencem a linhagens florestais, apresentem um forte sinal filogenético. Deste modo, os interflúvios planálticos do Cerrado parecem funcionar como uma barreira que previne, ou restringe, a co-ocorrência de linhagens com histórias biogeográficas distintas.

No terceiro capítulo, fazendo uso de modelos generalizados de dissimilaridade (GDM, Ferrier et al. 2007), avaliei o papel dos gradientes ambientais e condicionantes históricos nos padrões de beta diversidade entre taxocenoses de anuros do Cerrado. Para representar os fatores históricos, utilizei a semelhança no envelope ambiental (Graham et al. 2006) com os domínios vizinhos, com base na hipótese de que espécies pertencentes a linhagens mais associadas a outros domínios tenham a tendência de ocorrer nos ambientes mais semelhantes àqueles em que suas espécies-irmãs ocorrem (Ricklefs 2006). Os modelos produzidos revelaram que a dissimilaridade entre taxocenoses está associada a fatores tais como altitude, temperatura do mês mais frio, precipitação durante a estação seca, encharcamento do solo e semelhança no envelope ambiental com domínios vizinhos. Estes fatores estão relacionados tanto aos limites de tolerância fisiológica, quanto à disponibilidade de diferentes sítios reprodutivos no tempo e no espaço. Os modelos diferiram quando utilizei taxocenoses completas ou apenas espécies endêmicas, de modo que os fatores históricos foram mais importantes para as espécies endêmicas. Isso indica que espécies endêmicas parecem estar respondendo às condições ambientais baseadas na similaridade com os biomas vizinhos, onde suas espécies-irmãs ocorrem.

Os mapas resultantes dos GDMs diferiram de acordo com a base de dados utilizada. Nos mapas produzidos a partir das taxocenoses completas, observei uma correspondência com a subdivisão em zonas biogeográficas para espécies arbóreas (Ratter et al. 2003) e com as áreas de ocorrência de espécies e linhagens de anfíbios pertencentes aos diferentes grupos 
biogeográficos (Capítulo 1 e 2). Contudo, não há correspondência com os mapas de fisionomias vegetais (Silva et al. 2006), sugerindo que a ocorrência das espécies esteja mais limitada por fatores históricos do que pela distribuição das fitofisionomias. Os mapas resultantes dos modelos construídos a partir de espécies endêmicas diferiram na sua configuração. $O$ mapa resultante do GDM 3 (fatores locais e espécies endêmicas) subdivide o Cerrado de acordo com a topografia. Por outro lado, a configuração do mapa resultante do GDM 4 (fatores locais e históricos, e espécies endêmicas) apresentou uma forte influência da similaridade com o envelope ambiental dos domínios vizinhos. A partir desses resultados, pude observar que fatores ambientais locais e limitações relacionadas à história biogeográfica das linhagens se combinam para determinar a composição das taxocenoses de anuros no Cerrado.

De uma maneira sintética esta tese conclui que:

1. Metade das espécies de anuros que ocorrem no Cerrado é endêmica

2. As espécies que ocorrem no Cerrado e em um domínio vizinho; apresentam forte estruturação espacial, sendo limitadas às zonas mais próximas à área de contato com o domínio vizinho;

3. Espécies (mesmo que endêmicas do Cerrado) pertencentes a gêneros típicos de domínios florestais apresentam o mesmo tipo de estruturação: espécies pertencentes a gêneros atlânticos tendem a ocorrer em montanhas próximas ao limite com a Mata Atlântica, ao passo que espécies pertencentes a gêneros amazônicos tendem a ocorrer em vales próximos ao limite com a Amazônia;

4. Os interflúvios planálticos parecem funcionar como barreira à distribuição de espécies pertencentes a gêneros florestais;

5. A dissimilaridade entre as taxocenoses do Cerrado está associada tanto a fatores ambientais locais quanto à similaridade no envelope ambiental com as regiões onde outras espécies filogeneticamente relacionadas ocorrem.

\section{Referências}

Ferrier, S., G. Manion, J. Elith \& K. Richardson (2007). Using generalized dissimilarity modelling to analyse and predict patterns of beta diversity in regional biodiversity assessment. Diversity and Distributions 13: 252264. 
Gotelli, N. J. (2000). Null model analysis of species co-occurrence patterns. Ecology. 81: 2606-2621.

Graham, C. H., C. Moritz \& S. E. Williams (2006). Habitat history improves prediction of biodiversity in rainforest fauna. Proceedings of the National Academy of Sciences 103: 632-636.

Ratter, J. A., S. Bridgewater \& J. F. Ribeiro (2003). Analysis of the floristic composition of the Brazilian cerrado vegetation III: comparison of the woody vegetation of 376 areas. Edinburgh Journal of Botany 60: 57109.

Ricklefs, R. E. (2006). Evolutionary diversification and the origin of the diversity-environment relationship. Ecology 87: S3-S13.

Silva, J. F., M. R. Fariñas, J. M. Felfili \& C. A. Klink (2006). Spatial heterogeneity, land use and conservation in the cerrado region of Brazil. Journal of Biogeography 33: 536-548. 


\section{Anexos e Apêndices}

Anexo 1. Valdujo et al. 2009. Artigo publicado no periódico eletrônico Checklist.

Anexo 2. Vadujo et al. (no prelo). Artigo aceito para publicação no periódico Biota Neotropica em 2011. 


\title{
LISTS OF SPECIES
}

\section{Amphibia, Anura, São Desidério, western Bahia uplands, northeastern Brazil}

\author{
Paula Hanna Valdujo, ${ }^{1 *}$ Renato Sousa Recoder, ${ }^{2}$ Mariana Mira Vasconcellos, ${ }^{3}$ \\ and Alexandre de Souza Portella ${ }^{4}$ \\ ${ }^{1}$ Universidade de São Paulo, Instituto de Biociências, Departamento de Ecologia. \\ Rua do Matão, Travessa 14. CEP 055098-900. São Paulo, SP, Brazil. \\ ${ }^{2}$ Universidade de São Paulo, Instituto de Biociências, Departamento de Zoologia. \\ Rua do Matão, Travessa 14. CEP 055098-900. São Paulo, SP, Brazil. \\ ${ }^{3}$ University of Texas, Section of Integrative Biology. 78712. Austin, Texas, United States of America. \\ ${ }^{4}$ Universidade de Brasília, Instituto de Ciências Biológicas, Departamento de Ecologia. \\ Campus Universitário Darcy Ribeiro. CEP 70910-900. Brasília, DF, Brazil. \\ "Corresponding author. E-mail: paula.valdujo@gmail.com
}

\begin{abstract}
The contact zone between Cerrado and Caatinga is among the least known areas in the Cerrado biome, and no information is available about the anuran species occurring in this area and their ecology. Along this area, seasonally dry tropical forests are common on the lowlands with some enclaves inside the Cerrado core area. Here, we present for the first time a list of species on this zone recognized as a priority area for conservation inside the Cerrado. We sampled anuran species within $30 \mathrm{~km}$ of São Desidério, a city in western Bahia, from habitats including temporary and permanent ponds in Cerrado and seasonally dry tropical forests, as well as veredas and marshes. We recorded 32 anuran species, belonging to 12 genera in five families. Hylidae was the richest family (ten species) followed by Leiuperidae and Leptodactylidae (eight species each), Bufonidae (five species), and Microhylidae (two species). The heterogeneity of this site could be demonstrated by the presence of species with different dispersal/colonization history with eight species (25\%) endemic to Cerrado, three (9\%) shared with Caatinga biome, two (6\%) shared with Atlantic Forest, and eighteen $(56 \%)$ widespread through adjacent biomes.
\end{abstract}

\section{Introduction}

Geographical, evolutionary and historical processes in a region are important determinants of community assembly, influencing both local and regional diversity (Ricklefs and Schluter 1993). When located at the edge of a phytogeographic domain, the species composition of communities will be influenced by regional processes, like speciation, dispersal and immigration, both within the domain, and between adjacent domains (Lomolino et al. 2005). Therefore, the spatial dynamics of local communities may allow the coexistence of dispersing species from different regions with different abilities to use available resources in a heterogeneous landscape (Leibold et al. 2004).

The Cerrado is a large phytogeographic domain dominated by savanna vegetation located in the center of South America, bordering other open and forested biomes such as Caatinga, Atlantic Forest, Pantanal, Chaco and Amazonia. The contact zone between Cerrado and Caatinga extends largely through the states of Piauí, Bahia, and Minas Gerais in Brazil and represents one of the least known areas in the Cerrado biome. At the lowlands of this contact zone, seasonally dry tropical forest comprise the main vegetation type, especially through the São Francisco river basin, with some enclaves occurring inside the Cerrado core area in the states of Goiás and Tocantins (see description of vegetation aspects in Werneck et al. 2009). In western Bahia, seasonally dry forests are interspersed with typical Cerrado vegetation, resulting in a complex landscape providing suitable microhabitats for species with different ecological requirements.

Because of its broad area and high environmental heterogeneity, the Cerrado shows a significant 
turnover of amphibian species (Diniz-Filho et al. 2004). Based on the distribution of species in Cerrado, Diniz-Filho et al. (2007) stated that the peak of anuran richness occurs in central-southern region, decreasing toward the northeast. However, as suggested by Diniz-Filho et al. (2004), due to the uniqueness of the Cerrado / Caatinga contact zone in the western uplands of Bahia, this region is identified as one of the 10 priority areas for amphibian conservation in Cerrado, based on species distribution modeling, site complementarity analysis, and conservation efficiency.

Herein, we contribute to the knowledge of the Cerrado amphibian diversity by presenting a list of anuran species collected in the eastern part of the municipality of São Desidério, state of Bahia, with comments on the geographic distribution and habitat use of each species. We expect our data will help future studies on the biogeography of the Cerrado / Caatinga contact zone.

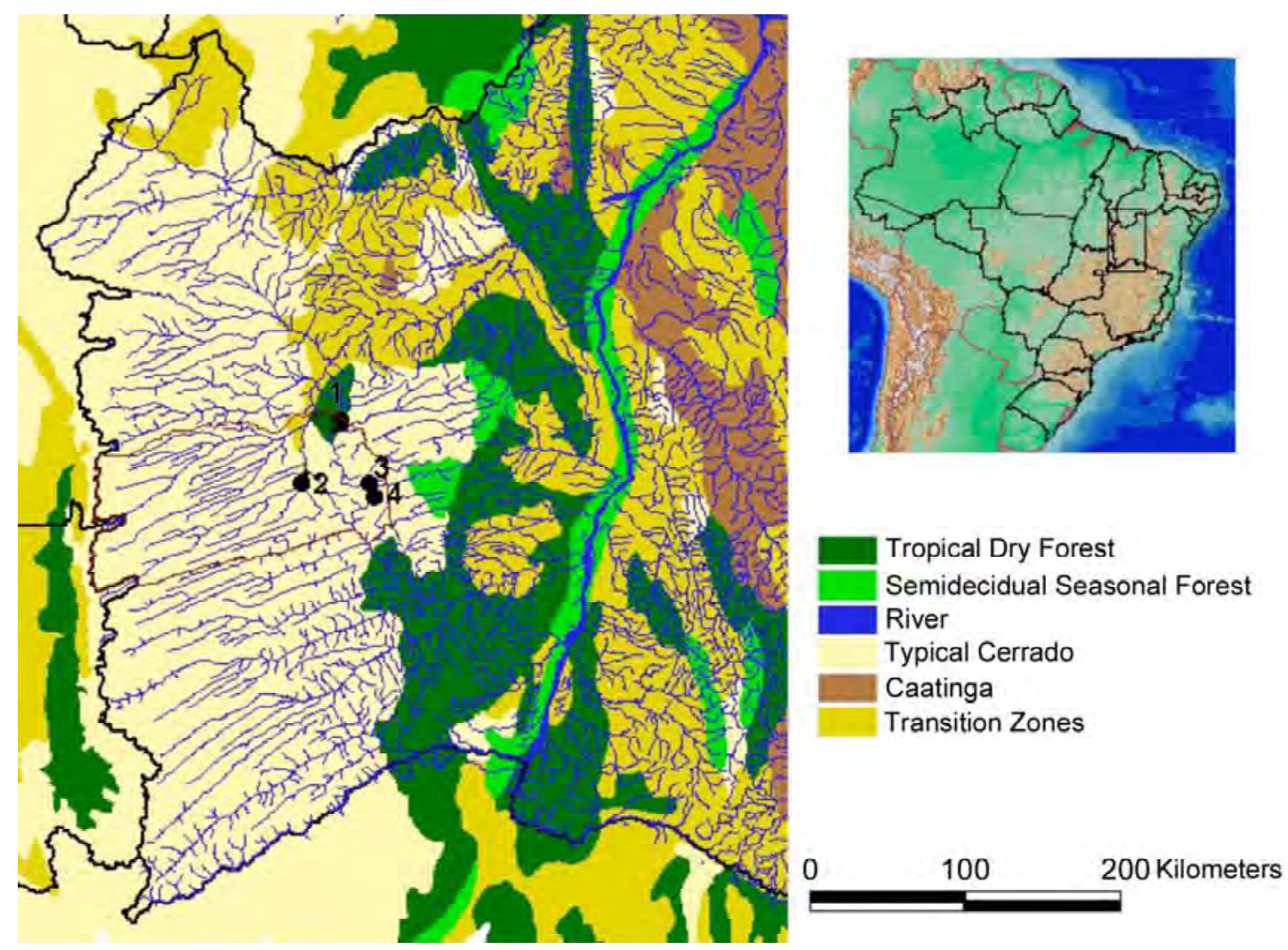

Figure 1. Sampled sites from the municipality of São Desidério, in western uplands of Bahia. Colors represent vegetation types (according to IBGE 2003). Numbers represent sampling sites: 1 - Parque Municipal Lagoa Azul and sorroundings; 2 - vereda do Anastácio; 3 - povoado de Campo Grande; 4 - temporary ponds at the municipalities of São Desidério and Correntina boundaries.

\section{Materials and Methods}

Study Site

The municipality of São Desidério is in Chapadão Ocidental da Bahia (the western uplands of Bahia), a flat, elevated plateau dominated by gently rolling grasslands on sandy interfluvial soils. Many springs flow through this region and drain into the São Francisco river. These tributaries run toward the east, almost parallel to each other, and reach a contact zone between seasonally dry tropical forests and savanna vegetation, in the lower altitudes of the eastern part of São Desidério. Calcareous rock outcrops are frequent through areas with a more steep relief. The western uplands of Bahia is among the most intensely exploited regions of the Cerrado, with high levels of habitat loss, especially due to the mechanized agricultural production of soybeans, cotton and corn. Collecting efforts were concentrated within $30 \mathrm{~km}$ on the eastern side of São Desidério. The following sites were sampled (see Figures 1 and 2):

1. Parque Municipal Lagoa Azul and surrounding areas: seasonally dry tropical forest area extending from the city of São Desidério to Parque 
Municipal Lagoa Azul. Temporary ponds and flooded areas close to the São Desidério river were sampled, as well as marshy areas formed right after heavy rains $\left(12^{\circ} 22^{\prime} \mathrm{S}, 44^{\circ} 51^{\prime} \mathrm{W}\right)$. Although we registered some amphibian species inside the park, especially close to the Gruta do Catão, we did not collect any specimens inside the protected area.

2. Rio Grande and Vereda do Anastácio marginal ponds: ponds, seasonally flooded grasslands and adjacent typical cerrado patches along Rio Grande $\left(12^{\circ} 42^{\prime} \mathrm{S}, 45^{\circ} 05^{\prime} \mathrm{W}\right)$.

3. Temporary and permanent ponds close to povoado de Campo Grande, along the recently paved road from the municipality of Correntina to São Desidério $\left(12^{\circ} 44^{\prime} \mathrm{S}, 44^{\circ} 41^{\prime} \mathrm{W}\right)$.

4. Temporary ponds close to the boundary of Correntina and São Desidério municipalities, along the recently paved road from Correntina to São Desidério, 20-30 kilometers south from site 3 $\left(13^{\circ} 01^{\prime} \mathrm{S}, 44^{\circ} 36^{\prime} \mathrm{W}\right)$.

\section{Data collection}

We conducted two field trips to São Desidério, one at the beginning of the rainy season, from October 31 to November 6, 2007, and a second trip in the peak of the rainy season, from January 15 to 19,2008 . All areas were sampled at least twice in each field trip. Amphibians were sampled at night by active search (visual and acoustic), which is considered the most efficient way to get the largest number of species in the least amount of time. This method involves tracing each call to its source and capturing at least one voucher specimen of each species by hand from all possible microhabitats. (Scott-Jr 1994). Voucher specimens were collected (Permit number 02001.001238/2007-52) and deposited at Coleção Célio Fernandes Baptista Haddad - Universidade Estadual Paulista, Campus Rio Claro (CFBH) and Coleção Herpetológica da Universidade de Brasília (CHUNB).

\section{Results and Discussion}

We found 32 anuran species, belonging to 12 genera in five families. Hylidae was the richest family (ten species) followed by Leiuperidae and Leptodactylidae (eight species each), Bufonidae (five species), and Microhylidae (two species). Eight species $(25 \%)$ are endemic to Cerrado, three $(9 \%)$ are shared with Caatinga biome, two (6\%) shared with Atlantic Forest, and eighteen $(56 \%)$ are widespread through adjacent biomes (Table 1, Figures 3 to 5).
Twenty-six of the 32 species were found calling and six other species were collected only by visual search. These may reproduce in non-sampled sites, in a non-sampled time of the year, or they may call only under special climatic conditions. Although not present in our list, Pseudopaludicola saltica, found in the adjacent municipalities of Barreiras and Jaborandi, could also occur in São Desidério.

This is the first record of Leptodactylus sertanejo apart from its type locality, in municipality of Uberlândia, state of Minas Gerais (Giaretta and Costa 2007). Our record extends its distribution more than $750 \mathrm{~km}$ to the northeast. We also fill gaps in the distribution of the recently described Rhinella veredas and $R$. cerradensis (see distribution maps in Brandão et al. 2007 and Maciel et al. 2007, respectively). Additionally, we add two new records to the Cerrado species list in Colli et al. (2002), Physalaemus albifrons and Pleurodema diplolistris. Until now, both of them were considered typical of the Caatinga biome.

Although the western uplands of Bahia do not show high levels of endemism (Diniz-Filho et al. 2007; this study) or diversity like other localities in the southeastern Cerrado (Diniz-Filho et al. 2004), they represent an important area in this biome as they harbor many species unique to this region occurring in the threatened seasonally dry forest and shared between Cerrado and Caatinga. This confirms the selection of this region as a priority area for conservation by a simple "greedy" algorithm based on area complementarity (Diniz-Filho et al. 2004).

Most of the registered species are habitat generalists, such as Leptodactyus cf. chaquensis, L. troglodytes, Phyllomedusa azurea, and Hypsiboas albopunctatus. However, we found some forest-associated species, such as Rhinella cf. pombali and Leptodactylus mystaceus, and grassland or other open area specialists such as Leptodactylus sertanejo and Rhinella veredas.

Marked seasonality and high spatial heterogeneity are two aspects that have profound effects on the life history and distribution of anurans in Cerrado habitats (Colli et al. 2002). Thus, local communities can harbor widespread species with varied ecological requirements occurring in Cerrado and adjacent biomes as observed in São Desidério. 
Table 1. List of species sampled from São Desidério with sites where each was found, collecting method and habitat type. $\mathrm{e}=$ Cerrado endemic; $\mathrm{w}=$ widespread; $\mathrm{ca}=$ Cerrado and Caatinga inhabitant; af $=$ Cerrado and Atlantic Forest inhabitant. DF = ponds or temporary lakes in dry forest; VE = vereda and associated marshes; GR $=$ grasslands, $\mathrm{TP}=$ temporary ponds in open areas.

\begin{tabular}{|c|c|c|c|}
\hline Amphibian species & Sites & Habitat & Method \\
\hline \multicolumn{4}{|l|}{ Family Bufonidae (5) } \\
\hline Rhinella cerradensis ${ }^{\mathrm{e}}$ Maciel, Brandão, Campos and Sebben, 2007 & 2 & $\mathrm{VE}$ & visual \\
\hline Rhinella cf. pombali ${ }^{\text {af }}$ & 1 & $\mathrm{DF}$ & acoustic \\
\hline Rhinella mirandaribeiroi ${ }^{\mathrm{w}}$ (Spix, 1824) & 234 & $\mathrm{TP}$ & acoustic \\
\hline Rhinella schneideri ${ }^{\mathrm{w}}$ (Werner, 1894) & 1234 & TP DF VE & acoustic \\
\hline Rhinella veredas ${ }^{\mathrm{e}}$ (Brandão, Maciel, and Sebben, 2007) & 234 & $\mathrm{VE}$ & visual \\
\hline \multicolumn{4}{|l|}{ Family Hylidae (10) } \\
\hline Dendropsophus minutus ${ }^{\mathrm{w}}$ (Peters, 1872) & 13 & $\mathrm{TP}$ & acoustic \\
\hline Dendropsophus nanus ${ }^{\mathrm{w}}$ (Boulenger, 1889) & 1234 & DF TP VE & acoustic \\
\hline Dendropsophus rubicundulus ${ }^{\mathrm{e}}$ (Reinhardt and Lütken, 1862) & 23 & $\mathrm{TP}$ & acoustic \\
\hline Dendropsophus melanargyreus ${ }^{\mathrm{w}}($ Cope, 1887$)$ & 1 & $\mathrm{DF}$ & acoustic \\
\hline Hypsiboas albopunctatus $^{\mathrm{w}}$ (Spix, 1824) & 123 & VE & acoustic \\
\hline Hypsiboas crepitans $^{\mathrm{w}}$ (Wied-Neuwied, 1824) & 14 & DF & acoustic \\
\hline Hypsiboas raniceps ${ }^{\mathrm{w}}$ Cope, 1862 & 14 & $\mathrm{TP}$ & acoustic \\
\hline Phyllomedusa azurea ${ }^{\mathrm{e}}$ Cope, 1862 & 1234 & DF TP & acoustic \\
\hline Scinax fuscomarginatus ${ }^{\mathrm{w}}$ (Lutz and Lutz, 1939) & 124 & TP VE & acoustic \\
\hline Scinax sp. gr. ruber & 124 & TP VE & acoustic \\
\hline \multicolumn{4}{|l|}{ Family Leiuperidae (8) } \\
\hline Eupemphix nattereri ${ }^{\mathrm{e}}$ Steindachner, 1863 & 14 & DF TP & acoustic \\
\hline Physalaemus albifrons $^{\mathrm{ca}}$ (Spix, 1824) & 2 & $\mathrm{VE}$ & visual \\
\hline Physalaemus centralis ${ }^{\mathrm{e}}$ Bokermann, 1962 & 24 & TP VE & acoustic \\
\hline 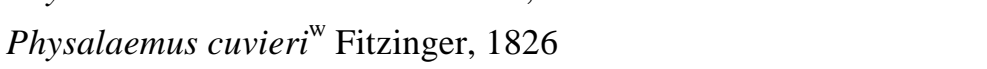 & 2 & TP VE & acoustic \\
\hline Physalaemus marmoratus ${ }^{\text {af }}$ (Reinhardt and Lütken, 1862) & 34 & $\mathrm{TP}$ & acoustic \\
\hline Pleurodema diplolister $^{\mathrm{ca}}$ (Peters, 1870) & 12 & TP GR & visual \\
\hline Pseudopaludicola ternetzi $i^{\mathrm{e}}$ Miranda-Ribeiro, 1937 & 2 & TP VE & acoustic \\
\hline \multicolumn{4}{|l|}{ Family Leptodactylidae (8) } \\
\hline Leptodactylus hylaedactylus ${ }^{\mathrm{w}}$ (Cope, 1868) & 1 & DF & acoustic \\
\hline Leptodactylus fuscus ${ }^{\mathrm{w}}$ (Schneider, 1799) & 124 & DF VE TP GR & acoustic \\
\hline Leptodactylus labyrinthicus ${ }^{\mathrm{w}}$ (Spix, 1824) & 4 & $\mathrm{VE}$ & acoustic \\
\hline Leptodactylus cf. chaquensis ${ }^{\mathrm{w}}$ & 1234 & DF TP VE & visual \\
\hline Leptodactylus mystaceus $^{\mathrm{w}}$ (Spix, 1824) & 14 & DF & acoustic \\
\hline Leptodactylus podicipinus $^{\mathrm{w}}$ (Cope, 1862) & 14 & DF TP & acoustic \\
\hline Leptodactylus sertanejo ${ }^{\mathrm{e}}$ Giaretta and Costa, 2007 & 2 & GR & acoustic \\
\hline Leptodactylus troglodytes ${ }^{\mathrm{ca}}$ Lutz, 1926 & 234 & DF TP & acoustic \\
\hline \multicolumn{4}{|l|}{ Family Microhylidae (2) } \\
\hline Dermatonotus muelleri $^{\mathrm{w}}$ (Boettger, 1885) & 1 & $\mathrm{DF}$ & visual \\
\hline Elachistocleis ovalis $^{\mathrm{w}}$ (Schneider, 1799) & 4 & TP VE & acoustic \\
\hline
\end{tabular}



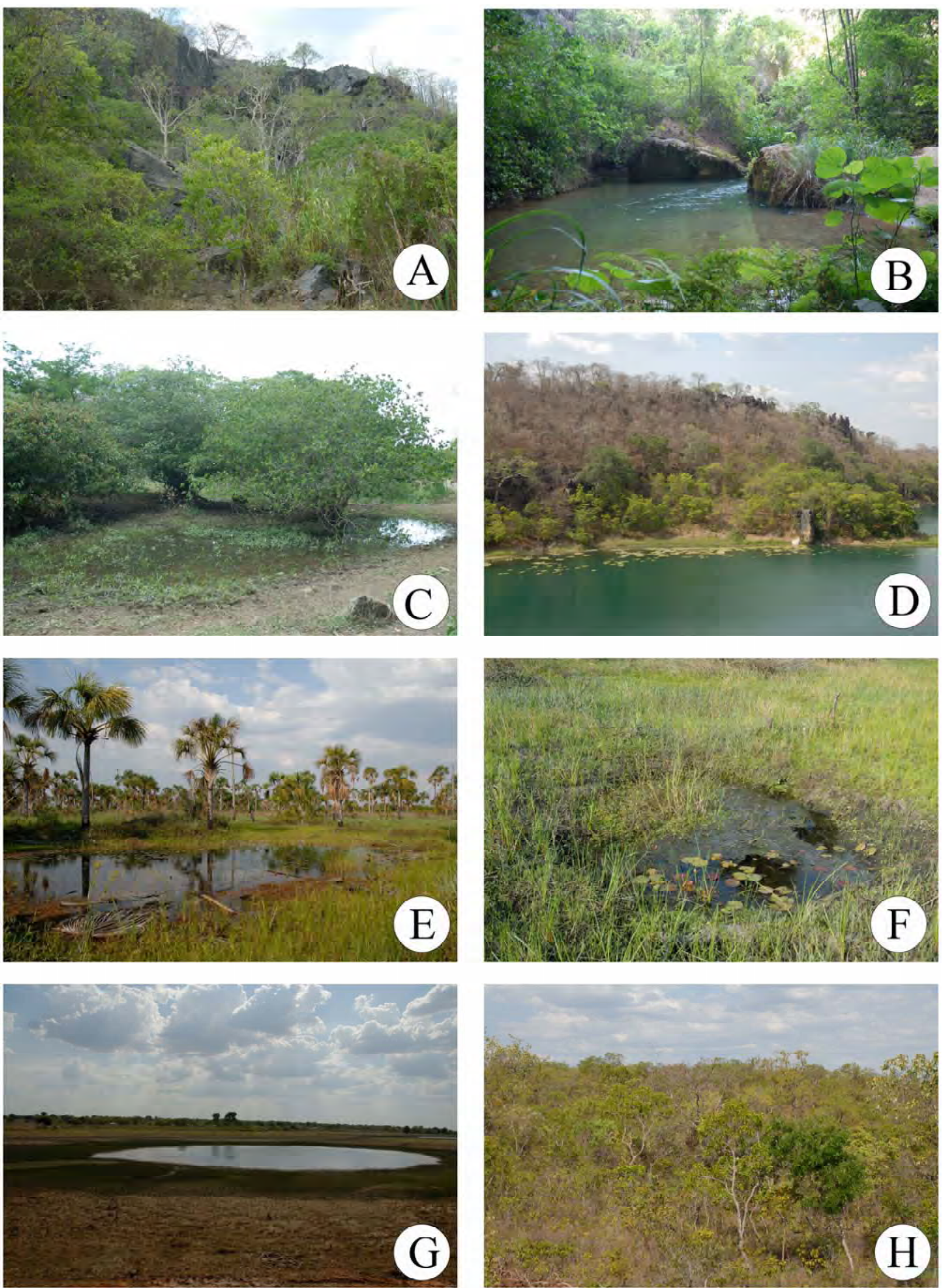

Figure 2. Sampling sites at São Desidério, state of Bahia. A. Rock outcrops and seasonally dry forest; B. Permanent pond at Parque Municipal Lagoa Azul; C. Temporary pond at São Desidério river flooded margins; D. Sinkhole and temporary dry forest at Parque Municipal Lagoa Azul; E. Vereda do Anastácio; F. Vereda do Anastácio; G. Permanent pond at Campo Grande; H. Typical cerrado crossed by the road to Correntina. Photo: P. H. Valdujo. 

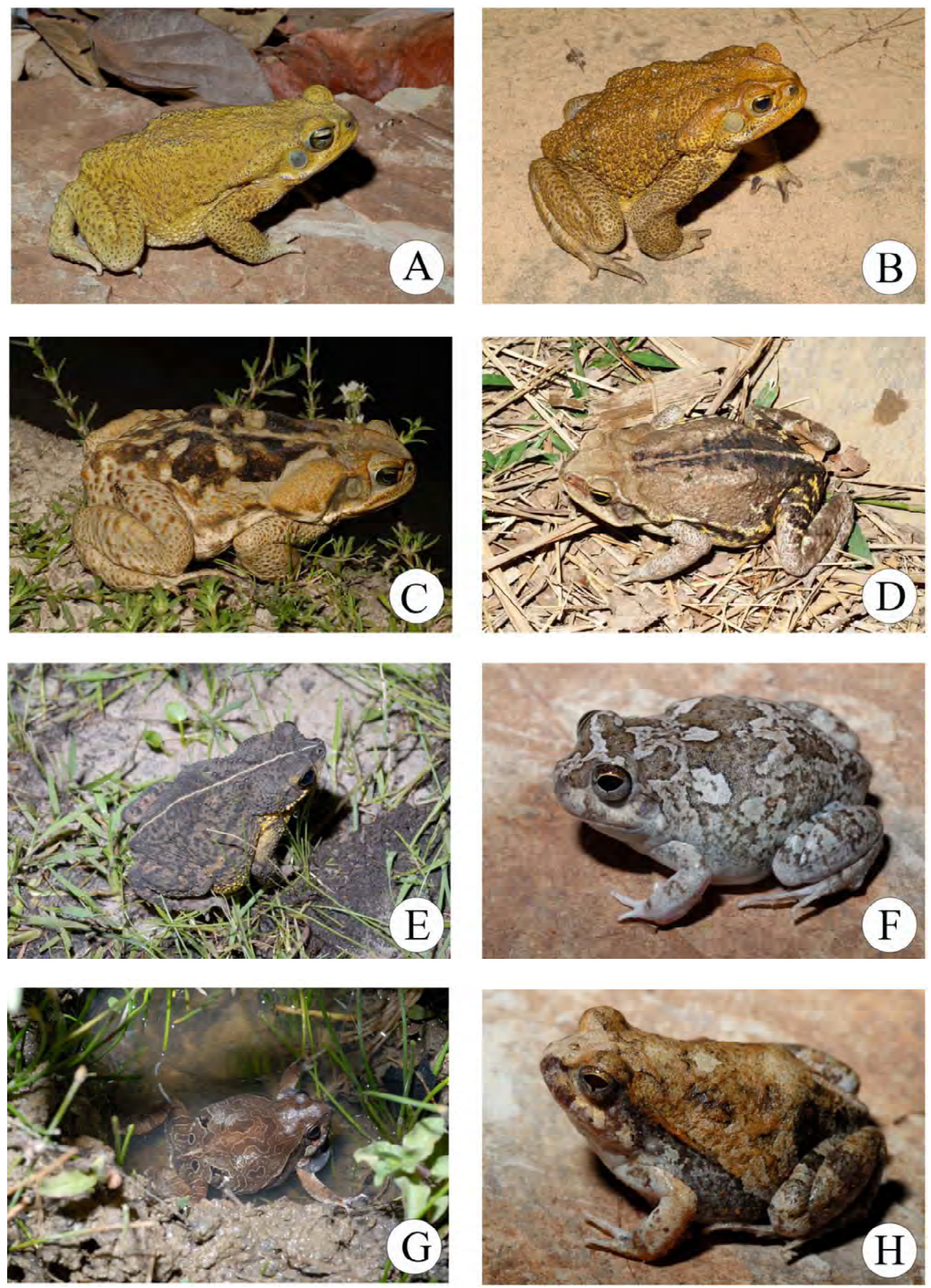

Figure 3. Bufonid species: A. Rhinella veredas; B. Rhinella cerradensis; C. Rhinella schneideri; D. Rhinella cf. pombali; E. Rhinella mirandaribeiroi; Leiuperid species: F. Pleurodema diplolister; G. Physalaemus centralis; H. Physalaemus albifrons; collected in the municipality of São Desidério, state of Bahia. Photo: P. H. Valdujo. 

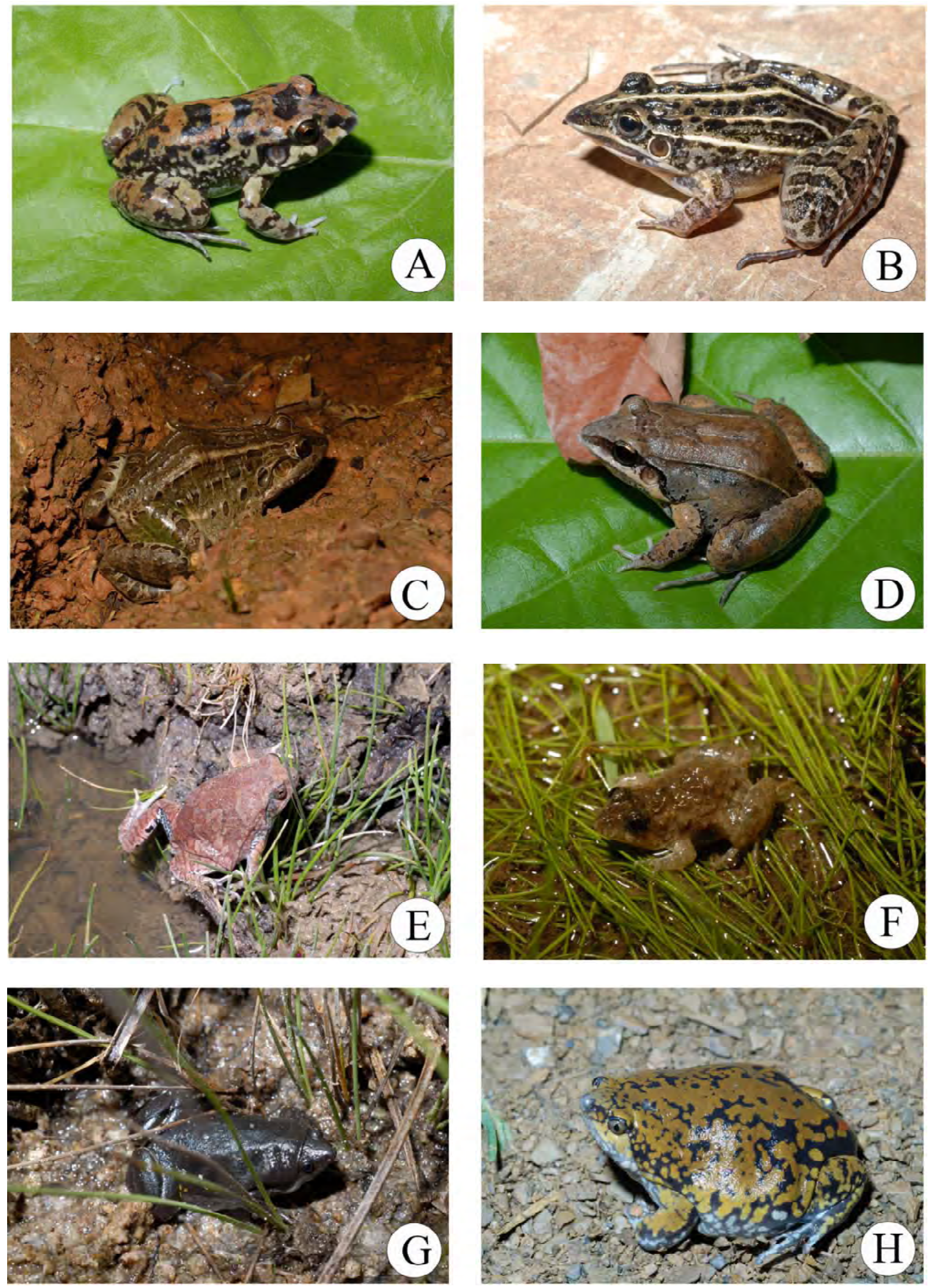

Figure 4. Leptodactylid species: A. Leptodactylus troglodytes; B. Leptodactylus sertanejo; C. Leptodactylus cf. chaquensis; D. Leptodactylus mystaceus. Leiuperid species: E. Eupemphix nattereri; F. Pseudopaludicola ternetzi. Microhylid species G. Elachistocleis ovalis; H. Dermatonotus muelleri; collected in the municipality of São Desidério, state of Bahia. Photo: P. H. Valdujo. 

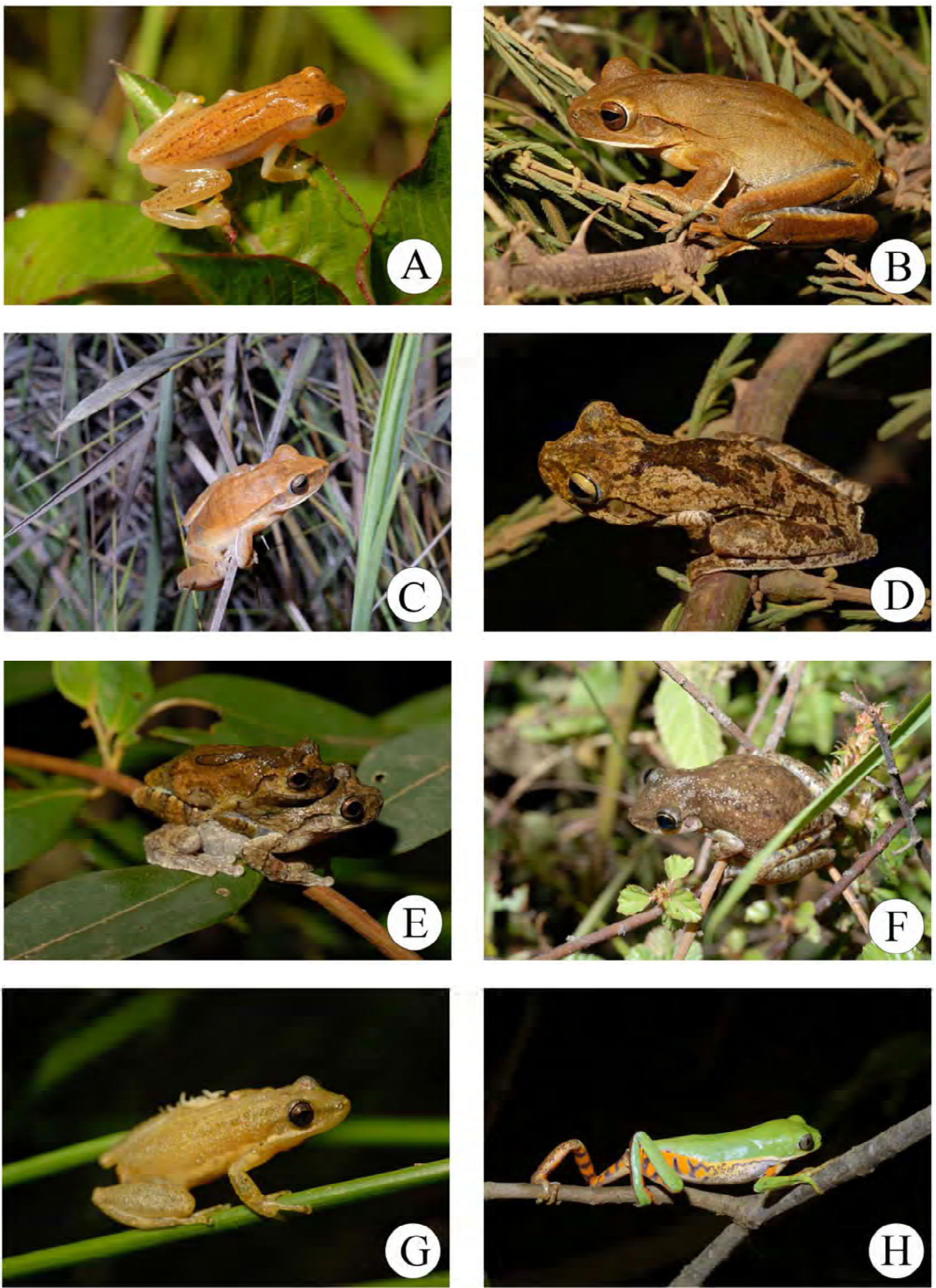

Figure 5. Hylid species collected in the municipality of São Desidério, state of Bahia. A. Dendropsophus nanus; B. Hypsiboas raniceps; C. Hypsiboas albopunctatus; D. Hypsiboas crepitans; E. Dendropsophus melanargyreus; F. Scinax sp. gr. ruber; G. Scinax fuscomarginatus; H. Phyllomedusa azurea. Photo: P. H. Valdujo. 
Acknowledgments: We thank Edson Cardoso, who drove us through previously unknown ways in western Bahia uplands, and Juscelino, who showed us some of the sampling sites and helped on frog collecting. Mr. Orlando allowed us to collect inside his farm at Vereda do Anastácio. Reuber Brandão confirmed the identification of Rhinella spp. Mark Brinkman critically read the manuscript and revised the English version. We are also grateful to Pequi - Pesquisa $e$ Conservação do Cerrado, Bioeste and Camila Vianello Bastazini for their helpful advices and logistic support, Natural Grasslands Conservancy, for the Student Grant awarded to PHV, and FAPESP for the fellowship provided to PHV.

\section{Literature cited}

Brandão, R.A., N.M. Maciel and A. Sebben. 2007. A new species of Chaunus from Central Brazil (Anura; Bufonidae). Journal of Herpetology 41(2): 309-316.

Colli, G.R., R.P. Bastos and A.F.B. Araújo. 2002. The character and dynamics of the Cerrado herpetofauna; p. 223-241 In P.S. Oliveira and R.J. Marquis (ed.). The Cerrados of Brasil: Ecology and Natural History of a Neotropical Savanna. Columbia: Columbia University Press.

Diniz-Filho, J.A.F., L.M. Bini, C.M. Vieira, M.C. Souza, R.P. Bastos, D. Brandão and L.G. Oliveira. 2004. Spatial patterns in species richness and priotity areas for conservation of anurans in the Cerrado region, Central Brazil. Amphibia-Reptilia 25: 63-75.

Diniz-Filho, J.A.F., L.M. Bini, M.P. Pinto, T.F.L.V.B. Rangel, P. Carvalho, S.L. Vieira and R.P. Bastos. 2007. Conservation biogeography of anurans in Brazilian Cerrado. Biodiversity and Conservation 16: 997-1008.

Giaretta, A.A. and H.C.M. Costa. 2007. A redescription of Leptodactylus jolyi Sazima and Bokermann (Anura, Leptodactylidae) and the recognition of a new closely related species. Zootaxa 1608: 1-10.

IBGE (Instituto Brasileiro de Geografia e Estatística). 2003. Mapa de Vegetação do Brasil. Escala 1:5.000.000. Rio de Janeiro: IBGE. $3^{\mathrm{a}}$ edição.

Leibold, M.A., M. Holyoak, N. Mouquet, P. Amarasekare, J.M. Chase, M.F. Hoopes, R.D. Holt, J.B. Shurin, R. Law, D. Tilman, M. Loreau and A.
Gonzalez. 2004. The metacommunity concept: a framework for multi-scale community ecology. Ecology Letters 7: 601-613.

Lomolino, M.V., B.R. Riddle and J.H. Brown. 2005. Biogeography, 3rd ed. Sunderland: Sinauer Associates. 845 p.

Maciel, N.M., R.A. Brandão, L.B. Campos and A. Sebben. 2007. A large new species of Rhinella (Anura: Bufonidae) from Cerrado of Brazil. Zootaxa 1627: 23-39.

Ricklefs, R.E. and D. Schluter. 1993. Species diversity: regional and historical influences; p. 350-363 In R.E. Ricklefs and D. Schluter (ed). Species Diversity in Ecological Communities: Historical and Geographical Perspectives. Chicago: The University of Chicago Press.

Scott-Jr, N.J. 1994. Complete Species Inventories; $p$ 78-84 In W.R. Heyer, M.A. Donnelly, R.W. McDiarmid, L.C. Hayek and M.S. Foster (ed). Measuring and Monitoring Biological Diversity Standard Methods for Amphibians. Washington: Smithsonian Intitution Press.

Werneck, F.P., G.R. Colli and L.J. Vitt. 2009. Determinants of assemblage structure in Neotropical dry forest lizards. Austral Ecology 34: 97-115.

Received: June 2009

Revised: September 2009

Accepted: November 2009

Published online: December 2009

Editorial responsibility: Marcelo N. de C. Kokubum

Appendix 1. Voucher specimens

Rhinella cerradensis (CHUNB 51113); Rhinella cf. pombali (CHUNB 51109), R. granulosa (CFBH 20544); $R$. schneideri (CHUNB 51115); R. veredas (CFBH 20516); Dendropsophus minutus (CFBH 20536); D. nanus (CFBH 20564); D. rubicundulus (CFBH 20533); D. melanargyreus (CFBH 20518); Hypsiboas albopunctatus (CHUNB 50989); H. crepitans (CFBH 20526); H. raniceps (CFBH 20525); Phyllomedusa azurea (CFBH 20557); Scinax fuscomarginatus (CFBH 20537); Scinax sp gr ruber (CFBH 20563); Eupemphix nattereri (CFBH 20531); Physalaemus albifrons (CHUNB 51061) P. centralis (CFBH 20539); P. marmoratus (CFBH 20562); Pleurodema diplolistris (CHUNB); Pseudopaludicola ternetzi (CHUNB); Leptodactylus fuscus (CFBH 20566); L. labyrinthicus (CHUNB 51138); L. chaquensis (CFBH 20514); L. mystaceus (CFBH 20555); L. podicipinus (CHUNB 51037); L. sertanejo (CFBH 20558); L. troglodytes (CFBH 20532); Dermatonotus muelleri (CFBH 20529); Elachistocleis ovalis (CFBH 20565). 


\title{
Anfíbios da Estação Ecológica Serra Geral do Tocantins, região do Jalapão, estados do Tocantins e Bahia
}

Título resumido: Anfíbios da EESGT, Jalapão, Brasil.

Paula Hanna Valdujo ${ }^{1 *}$, Agustín Camacho ${ }^{2}$, Renato Sousa Recoder ${ }^{2}$, Mauro Teixeira Junior $^{2}$, José Mário B. Ghellere ${ }^{2}$, Tami Mott ${ }^{3}$, Pedro Murilo Sales Nunes ${ }^{2}$ Cristiano Nogueira $^{4}$ e Miguel Trefaut Rodrigues ${ }^{2}$

${ }^{1}$ Departamento de Ecologia, Instituto de Biociências, Universidade de São Paulo, Rua do Matão, Trav. 14, n 321, Cidade Universitária, CEP 05508-900, São Paulo, SP, Brasil.

${ }^{2}$ Departamento de Zoologia, Instituto de Biociências, Universidade de São Paulo, Caixa Postal 11.461, CEP 05422-970, São Paulo, SP, Brasil.

${ }^{3}$ Universidade Federal de Mato Grosso, Av. Fernando Correa da Costa s/n, Coxipó, CEP 78060-900, Cuiabá, MT, Brasil.

${ }^{4}$ Departamento de Zoologia, Universidade de Brasília, Campus Universitário Darcy Ribeiro. CEP 70910-900, Brasília, DF, Brasil.

*Autor para correspondência. Email: paula.valdujo@gmail.com

\section{Amphibians from Estação Ecológica Serra Geral do Tocantins, Jalapão region, Tocantins and Bahia states}

\begin{abstract}
The northern part of Cerrado is one of the biologically poorest known areas in the domain. Recent studies revealed richness values that are as high as those from other sites in the domain. The Estação Ecológica Serra Geral do Tocantins is located in a region known as Jalapão, in northeastern Cerrado, and it is included in the largest set of protected areas in the domain. We describe amphibian richness and species composition in EESGT, and discuss it in a biogeographic context. We also describe breeding sites focusing on phytophysionomy and types of water bodies. We sampled amphibians through active search and pitfall traps, during the peak of breeding season for most of the anuran species in Cerrado. We registered 36 species, which coupled with former studies results in a regional richness of 39 species known for Jalapão. After applying Jackknife estimator, we suggest a potential richness of 42 species for the EESGT. Most registered species are endemic or strongly associated to Cerrado, followed by species widespread in Brazil or South America, and those associated with Caatinga. Most species breed in temporary ponds located in open areas, although there are some forest specialists, and stream-breeding species.
\end{abstract}

Key Words: Amphibia, Anura, Gymnophiona, inventory, breeding sites, Cerrado.

\section{Resumo}

A porção norte do domínio do Cerrado é uma das áreas historicamente menos conhecidas com relação à sua biodiversidade. Recentemente, alguns estudos tem revelado valores de riqueza comparáveis a outras regiões dentro do domínio. A Estação Ecológica Serra Geral do Tocantins (EESGT) está localizada na região do Jalapão, porção nordeste do Cerrado, e faz parte do maior bloco de áreas protegidas 
neste domínio. Neste estudo descrevemos a riqueza e composição de espécies de anfíbios da EESGT, discutindo-as em um contexto biogeográfico e caracterizamos o uso de sítios reprodutivos pelas espécies de anfíbios registradas em relação às fitofisionomias e aos tipos de corpos d'água. Utilizamos os métodos de busca ativa e armadilhas de queda, no período considerado como o auge da estação reprodutiva para a maior parte das espécies do Cerrado. Foram registradas 36 espécies de anfíbios na EESGT, totalizando 39 espécies conhecidas para a região do Jalapão. Aplicando o estimador Jackknife, sugerimos uma riqueza potencial de 42 espécies para a EESGT. A maior parte das espécies registradas é endêmica ou fortemente associada ao Cerrado, seguidas pelas espécies de ampla distribuição no Brasil ou América do Sul. A maior parte da espécies se reproduz em poças temporárias localizadas em áreas abertas, embora existam espécies que ocorrem exclusivamente em matas de galeria e utilizem corpos d'água lóticos para se reproduzir.

Palavras-Chave: Amphibia, Anura, Gymnophiona, inventário, sítios reprodutivos, Cerrado.

\section{Introdução}

O Brasil possui a maior riqueza de anfíbios no mundo: 847 espécies de anuros divididas em 19 famílias (Frost 2009, SBH 2010). A maior parte desta elevada riqueza está na Floresta Atlântica (Haddad \& Prado 2005), mas valores altos de riqueza têm sido também encontrados na Amazônia (Azevedo-Ramos \& Galatti 2002) e, mais recentemente, no Cerrado (Colli et al. 2002, Diniz-Filho et al. 2004, Bastos 2007), onde ocorrem 141 espécies. Em escala local, essa diversidade é bastante variável, tendo sido registradas de 28 a 52 espécies em diferentes localidades após estudos de longo prazo (respectivamente, Brasileiro et al. 2005, Pavan \& Dixo 2004).

Diniz-Filho et al. (2004) afirmam que os maiores valores de riqueza de anuros no Cerrado são encontrados na porção centro-sul. Todavia, em outro estudo mais recente que analisa a distribuição das espécies em relação a sua data de descrição, os mesmo autores discutem que a riqueza na porção norte do Cerrado deve ser mais alta do que os últimos estudos tem revelado, uma vez que boa parte dos inventários realizados até o início da década concentravam-se na porção central e sul do bioma (Diniz-Filho et al.2005). Apenas recentemente foram publicadas listas de espécies para a região centro-norte do Cerrado como para a UHE Luís Eduardo Magalhães, em Palmas, Tocantins (Pavan \& Dixo 2004), Parque Estadual do Jalapão (Vitt et al. 2005), do vale do rio Tocantins, entre os estados do Tocantins e Maranhão (Brasileiro et al. 2007) e para a região de Balsas no Maranhão (Barreto et al. 2007), revelando valores de riqueza maiores ou no mínimo comparáveis a outros locais dentro do domínio, e confirmando as hipóteses de Diniz-Filho et al. (2005). 
A fauna de anfíbios do Cerrado é composta por um número significativo (cerca de 30\%) de espécies endêmicas do bioma (Colli et al. 2002), por espécies típicas de domínios florestais (Mata Atlântica e Amazônia), que ocorrem principalmente em matas de galeria no Cerrado (Brandão \& Araújo 2001, Colli et al. 2002, Rodrigues 2005) e por espécies generalistas de áreas abertas. A distribuição de cada espécie é influenciada por diversos fatores históricos e atuais, que resultam em diferenças na composição de espécies entre localidades.

A exemplo do que se observa em relação à distribuição em escala regional, a distribuição das espécies entre hábitats na escala local também ocorre de forma heterogênea (exemplos em Brasileiro et al. 2005 e Uetanabaro et al. 2007). Os ambientes em que cada espécie ocorre são determinados por sua história de vida, sendo fortemente influenciados por suas estratégias para evitar predadores e encontrar ambientes adequados para reprodução (Wells 2007). Deste modo, em diversas localidades do domínio do Cerrado já foram descritos padrões de associação entre as espécies presentes e os ambientes disponíveis (Brasileiro et al. 2005, Uetanabaro et al. 2007, Araújo et al. 2009), podendo ser identificadas espécies típicas de formações florestais ou abertas, de corpos d'água permanentes ou temporários, lênticos ou lóticos.

Sabe-se que a maior parte das 31 espécies conhecidas para o Parque Estadual do Jalapão, próximo à Estação Ecológica Serra Geral do Tocantins, onde este estudo foi realizado, reproduzem-se em poças temporárias durante a estação chuvosa (Vitt et $a l .$, 2005). Contudo, não há informações detalhadas a respeito de como cada uma destas espécies utiliza o ambiente, ou da diversidade de sítios reprodutivos utilizados. Assim, este estudo visou ampliar o conhecimento a respeito da fauna de anfíbios da região do Jalapão, por meio de inventários realizados em áreas previamente não exploradas localizadas na Estação Ecológica Serra Geral do Tocantins e seu entorno. De maneira mais específica, este estudo teve como objetivos: 1) descrever a riqueza e composição de espécies de anfíbios da EESGT, discutindo-as em um contexto biogeográfico; 2) caracterizar o uso de sítios reprodutivos pelas espécies de anfíbios registradas em relação às fitofisionomias e aos tipos de corpos d'água.

\section{Material e Métodos}

1. Área de estudo 
A Estação Ecológica Serra Geral do Tocantins (EESGT) está localizada nos municípios de Mateiros, Almas e Ponte Alta, estado do Tocantins, e Formosa do Rio Preto, Bahia. A EESGT cobre uma área de 716 mil hectares pertencente à região do Jalapão e ao maior bloco de unidades de conservação do Cerrado, que inclui as Áreas de Proteção Ambiental do Jalapão e Serra da Tabatinga, a Estação Ecológica Serra Geral do Tocantins, o Parque Estadual do Jalapão e o Parque Nacional das Nascentes do Parnaíba. A vegetação nessa região é caracterizada como um mosaico de formações típicas do domínio do Cerrado, predominando campos e cerrados, entrecortados por matas de galeria, veredas e campos úmidos adjacentes, geralmente associados a nascentes.

A EESGT pode ser dividida geograficamente em seis unidades de paisagem: planície do alto rio Ponte Alta; depressão do alto rio Manoel Alves; serra da Piabanha; depressão do médio rio Novo; serra do Cinzeiro e do Meio; e o chapadão ocidental da Bahia. Apenas os rios da porção leste, pertencente à unidade do chapadão ocidental da Bahia, drenam para o rio São Francisco, enquanto todos os demais rios correm para a bacia do rio Tocantins. Neste estudo concentramos as amostragens em três regiões da EESGT (Figura 1): a porção sul, nas depressões do alto rio Manoel Alves, entre 27 de janeiro e 5 de fevereiro de 2008; e as porções central e leste, respectivamente na bacia do rio Novo e sobre os Chapadões da Serra Geral da Bahia, entre 7 e 16 de fevereiro de 2008.

Foram amostrados oito locais na EESGT, aqui referidas como "pontos de amostragem". A delimitação de cada ponto de amostragem foi meramente geográfica, de modo que alguns pontos podem ter mais de um tipo de ambiente, aqui referidos como "sítios reprodutivos".

1. Córrego Tarrafa $\left(11^{\circ} 19^{\prime} \mathrm{S}, 46^{\circ} 59^{\prime} \mathrm{W}\right)$. Três sítios reprodutivos distintos: a mata de galeria estreita, que acompanha seu curso; um grande afloramento laterítico coberto por um filme d'água, e cortado por pequenas valas causadas por erosão natural das chuvas; e uma área mais extensa de campo de murunduns.

2. Lagoa temporária $\left(11^{\circ} 19^{\prime} \mathrm{S}, 47^{\circ} 00^{\prime} \mathrm{W}\right)$. Um sítio: campo sujo sazonalmente alagável.

3. Mata Paludosa ( $\left.11^{\circ} 16^{\prime} \mathrm{S}, 47^{\circ} 00^{\prime} \mathrm{W}\right)$. Dois sítios: mata de galeria com solo alagado e campo úmido marginal no entorno da EESGT. 
4. Vereda $\left(11^{\circ} 12^{\prime} \mathrm{S}, 46^{\circ} 53^{\prime} \mathrm{W}\right)$. Três sítios: campo úmido, vereda e mata de galeria sobre solo alagado na porção sul da EESGT.

5. Sequência de poças temporárias na beira da estrada ( $\left.11^{\circ} 20^{\prime} \mathrm{S}, 46^{\circ} 59^{\prime} \mathrm{W}\right)$. Um sítio: poças formadas pela chuva em áreas de pastagem no entorno da EESGT.

6. Lagoa do Jorge ( $\left.10^{\circ} 57^{\prime} \mathrm{S}, 46^{\circ} 44^{\prime} \mathrm{W}\right)$. Um sítio: lagoa natural permanente em área de vegetação campestre localizada na porção central da EESGT.

7. Córrego Sapão $\left(10^{\circ} 44^{\prime} \mathrm{S}, 46^{\circ} 11^{\prime} \mathrm{W}\right)$. Três sítios: área de inundação do córrego Sapão, formada por lagoas, brejos e veredas.

8. Mata do Galhão ( $\left.10^{\circ} 34^{\prime} \mathrm{S}, 46^{\circ} 10^{\prime} \mathrm{W}\right)$. Um sítio: mata de galeria extensa associada ao rio Galhão com poças marginais ao longo do curso do rio.

\section{Métodos de amostragem}

Amostramos os anfíbios principalmente por meio de procuras visuais e auditivas noturnas (Heyer et al. 1994), período em que a maioria das espécies está em atividade. Para minimizar os efeitos do horário e pequenas variações nas condições climáticas sobre a atividade dos anuros, visitamos os mesmos ambientes em horários diferentes e em dias mais e menos chuvosos. Registramos cada indivíduo e/ou agregação observados e caracterizamos o ambiente quanto a sua localização geográfica, tipo de corpo d'água e fitofisionomia. Os corpos d'água foram classificados como lênticos ou lóticos e a fitofisionomia como aberta ou florestal.

Como forma de complementar as procuras noturnas, realizamos buscas durante o dia, utilizamos armadilhas de queda, e incluímos indivíduos encontrados de modo fortuito. As armadilhas de queda instaladas próximas a corpos d'água são importantes complementos no inventário de anfíbios, especialmente para a captura de espécies de hábitos terrestres e fossoriais (Heyer et al. 1994). Foram instalados nove conjuntos de armadilhas, compostos de 20 baldes de 35L e dispostos em "Y" em cada região da EESGT, totalizando um esforço de 1560 baldes/dia na porção sul da EESGT e 1620 baldes/dia na porção leste. Os ambientes amostrados com armadilhas de queda estão descritos em Rodrigues et al. (2008).

Coletamos séries de espécimes-testemunho para todas as espécies. Os exemplares foram fixados em formalina $8 \%$, depois transferidos para álcool 70\% e tombados na coleção herpetológica do Museu de Zoologia da Universidade de São Paulo (MZUSP). Examinamos os espécimes tombados no Museu de Zoologia da 
Universidade de São Paulo e Coleção Herpetológica da Universidade de Brasília provenientes de estudos anteriores na região do Jalapão.

\section{Análises}

Avaliamos a acurácia das nossas estimativas de riqueza por meio de curvas de rarefação construídas para as regiões sul e leste, e para o total da amostragem na EESGT. As curvas foram construídas a partir de 10.000 aleatorizações de uma matriz contendo frequência de ocorrência de cada espécie por dia de coleta, e produzidas no programa EstimateS v.8.0.0 (Colwell 2006). A partir das aleatorizações, foram também calculados valores de riqueza esperada com base no estimador Jackknife. Incluímos na matriz o número de sítios reprodutivos em que cada espécies foi detectada detectadas a cada dia, independentemente do método de amostragem. Seguimos este procedimento porque o objetivo da análise consistiu e estimar a riqueza total da EESGT, e em todos os dias de coleta os mesmos métodos foram aplicados com esforços aproximadamente iguais. As agregações de indivíduos da mesma espécie foram consideradas como uma única ocorrência, de modo que a abundância de uma espécie em um dia é considerada maior que um apenas se esta espécie tiver sido registrada em mais de um ponto de amostragem.

Por fim, comparamos os valores de riqueza observado e estimado àqueles obtidos por outros estudos disponíveis na literatura no intuito de avaliar nossos resultados e caracterizar a variação da riqueza local na porção norte do Cerrado. Utilizamos ainda informações disponíveis na literatura e em bancos de dados on line para caracterizar a distribuição geográfica das espécies encontradas na EESGT e subsidiar as discussões biogeográficas.

\section{Resultados}

Registramos 36 espécies de anfíbios na EESGT e seu entorno (Tabela 1, Figuras 3, 4, 5 e 6). Considerando levantamentos anteriores na região do Jalapão, a riqueza obtida para todo o mosaico de unidades de conservação foi de 39 espécies de anfíbios. A curva de acúmulo de espécies construída para a EESGT (Figura 2A) apresenta uma tendência à estabilização, embora o estimador de riqueza Jackknife tenha indicado a ocorrência de e 42,6 \pm 2,1 espécies.

Adicionamos 15 espécies à lista previamente conhecida para a região do Jalapão: Barycholos ternetzi, Rhinella mirandaribeiroi, Proceratophrys goyana, 
Corythomantis greeningi, Dendropsophus cruzi, D. soaresi, Hypsiboas raniceps, Phyllomedusa azurea, Scinax constrictus, Scinax sp. gr. ruber, Eupemphix nattereri, Leptodactylus hylaedactylus, L. podicipinus, L. sertanejo, L. syphax (Tabela 1). Considerando apenas os resultados obtidos na EESGT, foram registradas 12 espécies pertencentes à família Hylidae, nove Leptodactylidae, seis Leiuperidae, quatro Bufonidae, dois Microhylidae e apenas um representante das famílias Brachycephalidae, Caecilidae, e Cycloramphidae. A tabela 2 mostra a riqueza e distribuição de espécies por famílias neste estudo e em publicações anteriores.

Das 36 espécies de anfíbios registradas na EESGT, 15 (41,6\%) são endêmicas ou estão fortemente associadas aos limites do Cerrado. Treze espécies $(33,3 \%)$ têm distribuição ampla no Brasil ou América do Sul e hábitos generalistas. Quatro espécies $(11,1 \%)$ ocorrem no Cerrado e na Caatinga e três $(8,3 \%)$ nas formações abertas da América do Sul (incluindo Cerrado, Caatinga e Chaco). Por fim, duas espécies (5,5\%) ocorrem no Cerrado e na Amazônia. Apresentamos os dados de distribuição geográfica de cada espécie na tabela 1.

Registramos 28 espécies apenas em áreas abertas, incluindo campos úmidos, lagoas e poças temporárias, enquanto oito espécies foram registradas apenas em formações florestais ou borda de mata, e três espécies foram encontradas tanto em ambientes abertos quanto florestais (Tabela 1). As espécies endêmicas do Cerrado encontradas no Jalapão ocorrem tanto em ambientes florestais (ex: Barycholos ternetzi, Scinax constrictus) quanto em áreas abertas (ex: Eupemphix nattereri, Leptodactylus sertanejo). Em todas as outras categorias de distribuição predominam espécies típicas de áreas abertas (Tabela 1). Com relação ao tipo de corpo d'água utilizado para reprodução, 26 espécies ocorrem em poças ou lagoas, cinco apenas em poças marginais de riachos, três em campos úmidos, três em riachos ou outro tipo de corpo d'água corrente, e duas espécies têm desenvolvimento direto, sendo independentes de corpos d'água para reprodução.

\section{Discussão}

A riqueza de espécies de anfíbios encontrada na EESGT foi superior aos valores apresentados por outros estudos de curta duração na região norte do Cerrado (Tabela 2). Estes estudos revelam valores de riqueza comparáveis à de áreas inventariadas na porção centro-sul (Tabela 2), confirmando as previsões de DinizFilho et al. (2005). Contudo, Pavan \& Dixo (2004) reportam uma riqueza superior ao 
valor obtido por nosso estudo, com 52 espécies para as regiões da UHE Luís Eduardo Magalhães, em Palmas, Tocantins, baseado em um estudo de longa duração. Estes resultados evidenciam uma forte influência da fauna amazônica nas planícies de inundação do Tocantins contribuindo para sua alta riqueza. Ainda, reforçam a necessidade de estudos intensivos e de longa duração em mais localidades na porção norte do Cerrado, para que seja possível avaliar de uma forma mais precisa a distribuição da riqueza ao longo de toda a extensão do domínio. Desta forma e de acordo com os resultados obtidos pelos estimadores de riqueza, esperamos que futuros esforços nas mesmas áreas deverão acrescentar mais espécies à lista.

Dos 15 novos registros de espécies para a região do Jalapão, oito representam reidentificações de espécies já apresentadas com outros nomes por Vitt et al. (2005): Hypsiboas raniceps foi determinada anteriormente como Hypsiboas sp.; Dendropsophus cruzi, como D. microcephalus; Scinax constrictus, como $S$. rostratus; Scinax sp. gr. ruber, como Scinax ruber; Proceratophrys goyana como Proceratophrys sp.; Rhinella mirandaribeiroi como Rhinella granulosa; Leptodactylus hylaedactylus, como L. andreae; e Leptodactylus sertanejo como L. furnarius. Deste modo, apenas sete das 15 espécies representam adições reais à riqueza de espécies conhecida para a região do Jalapão: Barycholos ternetzi, Corythomantis greeningi, Dendropsophus soaresi, Phyllomedusa azurea, Eupemphix nattereri, Leptodactylus podicipinus e L. syphax. O registro de novas espécies pode ser devido à expansão da área amostrada, ao fato deste estudo ter sido desenvolvido durante o pico da estação reprodutiva da maior parte das espécies de anuros do Cerrado (Bastos 2007), ou simplesmente devido ao aumento do esforço de amostragem, possibilitando o registro de mais espécies raras.

A distribuição das espécies entre as famílias é semelhante a outras taxocenoses no Cerrado (Tabela 2), com predomínio de espécies da família Hylidae, com 31 a $50 \%$ das espécies de cada localidade. A segunda família mais rica é Leptodactylidae com 10 a $25 \%$ das espécies, seguida por Leiuperidae, com 7 a $22 \%$, sendo que em algumas localidades essas duas famílias se alternam no valor da riqueza. Bufonidae é a quarta família em número de espécies em todas as localidades, com 7 a 16\% das espécies em cada área. Esse padrão se repete também em escala geográfica mais amplas, de modo que essas quatro famílias são as que apresentam o maior número de espécies no Cerrado (Bastos 2007). 
A composição de espécies encontrada pode estar relacionada à localização geográfica do Jalapão, dentro do domínio do Cerrado, mas próximo à área de transição com a Caatinga. Além disso, as áreas amostradas pertencem a duas importantes bacias hidrográficas, a do rio São Francisco na porção mais oriental e a do rio Tocantins, compreendendo toda a área a oeste da Serra Geral. Isso possibilita a coexistência de espécies tipicamente associadas ao Cerrado, tais como Dendropsophus rubicundulus, Rhinella ocellata, Barycholos ternetzi, com espécies consideradas típicas da Caatinga, tais como Corythomantis greeningi, Leptodactylus troglodytes e Pleurodema diplolister, e espécies pertencentes a linhagens amazônicas, tais como Scinax constrictus (pertencente ao grupo de S. rostratus), Osteocephalus cf. taurinus (registrado na região por Vitt et al. 2005) e Rhaebo guttatus. O predomínio de espécies endêmicas do Cerrado e de espécies com ampla distribuição no Brasil em relação às espécies compartilhadas entre o Cerrado e um único Bioma vizinho é consistente com o padrão observados em outros estudos no domínio do Cerrado (Uetanabaro et al. 2007, Brasileiro et al. 2007, Valdujo et al. 2009).

Na EESGT, o uso de ambientes abertos como sítios reprodutivos é mais comum do que o uso de ambientes florestais tanto para espécies endêmicas ou fortemente associadas ao Cerrado quanto para as que ocorrem em mais de um bioma. Esse fato pode estar relacionado à maior extensão das formações campestres e savânicas quando comparada às de áreas florestais no Cerrado (Oliveira-Filho \& Ratter 2002), apesar da diversidade de microhabitats para reprodução em áreas abertas ser, de um modo geral, menor que em florestas (Cardoso et al. 1989). A maior parte das espécies de áreas abertas encontradas na EESGT, e no Jalapão como um todo, se reproduz sazonalmente em poças temporárias, durante a estação chuvosa, e isso pode estar relacionado ao fato das espécies do Cerrado apresentarem reprodução sazonal, concentrada na estação chuvosa, com poucas exceções (Bastos, 2005).

Nossos resultados ampliam a distribuição conhecida de Phyllomedusa azurea e Barycholos ternetzi cerca de $200 \mathrm{~km}$ a sudeste de Palmas, a localidade mais próxima conhecida (Pavan \& Dixo, 2004); registram Corythomantis greeningi pela primeira vez no domínio do Cerrado; ampliam a distribuição de Leptodactylus sertanejo em cerca de $900 \mathrm{~km}$ a partir de sua localidade-tipo em Uberlândia, estado de Minas Gerais (Giaretta \& Costa 2007); e preenchem uma lacuna na distribuição de Leptodactylus syphax entre as populações do Planalto Central e os poucos registros disponíveis para a região nordeste do Brasil (ver mapa em Fernandez et al. 2009). 
Além disso, corroboram a hipótese de Diniz-Filho et al. (2005) de que a aparente baixa diversidade de espécies na porção norte do Cerrado é um reflexo de escassez de inventários. Reforçamos a necessidade de estudos intensivos concentrados no período de reprodução das espécies de anfíbios para a caracterização adequada da anurofauna regional. Ao passo que estudos de curto prazo permitem aumentar nosso conhecimento sobre a estrutura, requisitos ecológicos e estrutura das comunidades de anfíbios do Cerrado, estudos mais extensivos são necessários para compreender detalhadamente os padrões regionais de riqueza de espécies.

\section{Agradecimentos}

Agradecemos à Fundação O Boticario de Proteção à Natureza (projeto 0747 20071) e à Conservação Internacional (projeto CP FY08/07) pelo apoio financeiro, à Pequi Pesquisa e Conservação do Cerrado pelo apoio logístico, ao Instituto Chico Mendes de Conservação da Biodiversidade por permitir acesso à EESGT (autorização 121871), à FAPESP e ao CNPq pelas bolsas concedidas aos autores. Agradecemos a G.R. Colli, M.A. Brasil e M.C.M. Viana por permitir acesso aos espécimes depositados na Coleção Herpetológica da Universidade de Brasília, a H. Zaher e Carolina Castro Mello pelo acesso à coleção do Museu de Zoologia da Universidade de São Paulo e a D.L. Silvano pelo auxílio no exame dos exemplares e levantamento de informações de distribuição geográfica. Agradecemos a R. Sawaya, C. Brasileiro, e A. Eterovic, além de dois revisores anônimos pelas sugestões que aprimoraram o manuscrito.

\section{Referências Bibliográficas}

ARAUJO, C.O., T.H. CONDEZ \& SAWAYA, R.J. 2009. Anfíbios anuros do Parque Estadual das Furnas do Bom Jesus, sudeste do Brasil, e suas relações com outras taxocenoses no Brasil. Biota Neotropica 9(2):

http://www.biotaneotropica.org.br/v9n2/en/abstract?article+bn01309022009 (último acesso em 05/01/2010).

AZEVEDO-RAMOS, C. \& GALATTI, U. 2002. Patterns of amphibian diversity in Brazilian Amazonia: conservation implications. Biol Conserv 103(1):103-111.

BARRETO, L., C. ARZABE \& LIMA,Y.C.C. 2007. Herpetofauna da região de Balsas. In: Cerrado Norte do Brasil - North Cerrado of Brazil (L. Barreto, ed.). USEB, Pelotas, p. 221-229.

BASTOS, R. P. 2007. Anfíbios do Cerrado. In Herpetologia no Brasil II. 1 ed. (L. B. Nascimento; \& M. E. Oliveira, coord.). Sociedade Brasileira de Herpetologia, Belo Horizonte, v. 1, p. 87-100.

BRANDÃO E.A. \& ARAÚJO A.F.B. 2001. A herpetofauna associada às Matas de Galeria no distrito Federal. In Cerrado: caracterização e recuperação de Matas de Galeria (J. F. Ribeiro, C. E. L. da Fonseca \& J. C. Sousa-Silva, eds). Embrapa, Brasília, p. 561-606.

BRASILEIRO, C.A., R.J. SAWAYA, M.C. KIEFER \& MARTINS, M. 2005. Amphibians of an open Cerrado fragment in southeastern Brazil. Biota Neotropica 5(2): http://www.biotaneotropica.org.br/v5n2/pt/abstract?article+BN00405022005 (último acesso em 09/03/2009) 
BRASILEIRO, C.A., E.M. LUCAS, H.M. OYAMAGUCHI, M.T.C. THOMÉ \& DIXO, M. 2007. Anurans, Northern Tocantins River Basin, states of Tocantins and Maranhão, Brazil. CheckList 4(2):185-197.

CARDOSO, A.J., G.A. ANDRADE \& HADDAD, C.F.B. 1989. Distribuição espacial em comunidades de anfíibios (Anura) no Sudeste do Brasil. Revista Brasileira de Biologia 49: 241-249.

CINTRA, C.E.D., H.L.R. SILVA \& SILVA-JR. N.J. 2009. Herpetofauna, Santa Edwiges I and II hydroelectric power plants, state of Goiás, Brazil. Check List 5(3):570-573.

COLLI, G.R., BASTOS, R.P. \& ARAUJO. A.F.B. 2002. The character and dynamics of the Cerrado herpetofauna. In The Cerrados of Brazil: ecology and natural history of a neotropical savanna (P. S. Oliveira \& R. J. Marquis, eds.). Columbia University Press, New York, p. 223-239.

COLWELL, R.K. 2006. EstimateS: Statistical estimation of species richness and shared species from samples. Version 8.0. User's Guide and application published at: http://purl.oclc.org/estimates. (último acesso em 14/06/2009)

DINIZ-FILHO, J.A. F., BINI, L.M., VIEIRA, C.M., SOUZA, M.C., BASTOS, R.P., BRANDÃO, D. \& OLIVEIRA, L.G. 2004. Spatial patterns in species richness and priority areas for conservation of anurans in the Cerrado region, Central Brazil. Amphibia-Reptilia 25(1):63-75.

DINIZ-FILHO, J.A.F., R.P. BASTOS, T.F.L. V.B. RANGEL, L.M. BINI, P. CARVALHO \& SILVA, R.J. 2005. Macroecological correlates and spatial patterns of anuran description dates in the Brazilian Cerrado. Global Ecol Biogeogr 14(5):469-477.

FERNANDEZ, M., D. COLE, W.R. HEYER, S. REICHLE \& R.O. DE SÁ. 2009. Predicting Leptodactylus (Amphibia, Anura, Leptodactylidae) distributions: broad-ranging versus patchily distributed species using a presence-only environmental niche modeling technique. South American J Herpet 4(2):103-116.

FROST, D.R. Amphibian species of the world: an online reference. Version 5.3. http://research.amnh.org/herpetology/amphibia/. (último acesso em 12/02/2009).

GIARETTA, A.A. \& COSTA, H.C.M 2007. A redescription of Leptodactylus jolyi Sazima and Bokermann (Anura, Leptodactylidae) and the recognition of a new closely related species. Zootaxa 1608:1-10.

HADDAD C.F.B. \& C.P.A. PRADO 2005. Reproductive modes in frogs and their unexpected diversity in the Atlantic Forest of Brazil. Bioscience 55(3):207-217.

HEYER, W.R., DONNELLY, M.A., MCDIARMID, R.W., HAYEK L.C.\& FOSTER, M.S. 1994. Measuring and monitoring biological diversity: Standard Methods for Amphibians. Smithsonian Institution, Washington, $364 \mathrm{p}$.

OLIVEIRA-FILHO, A. \& RATTER, J. 2002. Vegetation Physiognomies and woody flora of the Cerrado biome. In The Cerrados of Brazil: Ecology and Natural History of a Neotropical Savanna (P.S. Oliveira \& R.J. Marquis). Columbia University Press. New York.

PAVAN, D. \& DIXO, M. 2004. A herpetofauna da área de influência do reservatório da Usina Hidrelétrica Luís Eduardo Magalhães, Palmas, TO. Humanitas 4(6):1330.

RODRIGUES M.T. 2005. A biodiversidade dos Cerrados: conhecimento atual e perspectivas, com uma hipótese sobre o papel das matas de galerias na troca faunística durante ciclos climáticos. In Cerrado: Ecologia, Biodiversidade e Conservação (A. Scariot, J. C. Sousa-Silva \& J. M. Felfili, coords.). Ministério do Meio Ambiente, Brasília, p. 234-246. 
RODRIGUES, M.T., A. CAMACHO, P.M.S. NUNES, R.S. RECODER, M. TEIXEIRA JR., P.H. VALDUJO, J.M.B. GHELLERE, T. MOTT \& NOGUEIRA, C. 2008. A new species of the lizard genus Bachia (Squamata: Gymnophthalmidae) from the Cerrados of Central Brazil. Zootaxa 1875: 39-50.

SBH. 2010. Brazilian amphibians - List of species. Accessible at http://www.sbherpetologia.org.br. Sociedade Brasileira de Herpetologia. (último acesso em 19/01/2011).

SILVA-JR., N.J., C.E.D. CINTRA, H.L.R. SILVA, M.C. COSTA, C.A. SOUZA, A.A. PACHECCO-JR, GONÇALVES, F.A. Herpetofauna, Ponte de Pedra hydroelectric power plant, states of Mato Grosso and Mato Grosso do Sul, Brazil. Check List 5(3):518-525.

UETANABARO, M., F.L. SOUZA, P. LANDGREF-FILHO, A.F. BEDA \& BRANDÃO, R.A. 2007. Anfíbios e répteis do Parque Nacional da Serra da Bodoquena, Mato Grosso do Sul, Brasil. Biota Neotropica 7(3): http://www.biotaneotropica.org.br/v7n3/pt/abstract?inventory+bn01207032007 (último acesso em 09/03/2009).

VALDUJO P.H., R.S. RECODER, M.M. VASCONCELOS \& PORTELLA, A.S. Amphibia, Anura, São Desidério. western Bahia uplands, northeastern Brazil. Check List 5(4):903-911.

VAZ-SILVA, W. A.G. GUEDES, P.L. AZEVEDO-SILVA, F.F. GONTIJO, R.S. BARBOSA, G.R.ALOÍSIO, F.C.G. OLIVEIRA. Herpetofauna, Espora hydroelectric power plant, state of Goiás, Brazil. Check List 3(4):338-345.

VITT, L.J., CALDWELL, J.P., COLLI, G.R., GARDA, A.A., MESQUITA, D.O., FRANÇA, F.G.R., SHEPARD, D.B., COSTA, G.C., VASCONCELLOS, M.M. \& de NOVAES-E-SILVA, V. 2005. Uma atualização do guia fotográfico de répteis e anfíbios da região do Jalapão no Cerrado Brasileiro. Special Publications in Herpetology, SNOMNH Occasional Papers 2:1-24.

WELLS, K.D. 2007. The Ecology and Behavior of Amphibians. The University of Chicago Press. Chicago. 


\section{Tabelas}

Tabela 1. Lista de espécies de anfíbios registradas na região do Jalapão, com informações a respeito do tipo de fitofisionomia e corpo d'água, distribuição geográfica e local de registro das espécies. Abreviações: PEJ - espécies registradas por Vitt et al. (2005) no Parque Estadual do Jalapão. Asteriscos indicam que os registros tiveram sua determinação modificada; EESGT - espécies registradas durante este estudo. Habitat: A - formações abertas; F - formações florestais. Tipo de corpo d'água: C - corpos d'água corrente; $\mathrm{M}$ - poças marginais a rios; $\mathrm{PT}$ - poças temporárias; UM - campo úmido. Distribuição Geográfica: AM - espécie com ocorrência no Cerrado e na Amazônia; CA - espécie registrada no Cerrado e na Caatinga; $\mathrm{E}$ - espécie endêmica do Cerrado; $\mathrm{O}$ - espécie distribuída pelas formações abertas da América do Sul (Cerrado, Caatinga e Chaco); T - espécies típicas do Cerrado, mas com ocorrência ocasional em domínios vizinhos; $\mathrm{W}$ - espécie generalista de ampla distribuição no Brasil ou América do Sul.

Table 1. List of amphibian species from Jalapão, including information on habitat type, type of water body, geographic distribution and places where each species was registered in Jalapão. PEJ - species registered by Viit et al. (2005) in Jalapão State Park, asterisks indicate that original identification was changed; "EESGT" - species registered during this study. Habitat type: A - open habitats; F - forest habitats. Type of water body: C - rivers or streams; M - marginal ponds; PT - temporary ponds; UM - wetlands. Geographic distribution: AM - species registered in Cerrado and Amazonia; CA - species registered in Cerrado and Caatinga; E - endemic species; O species occurring in South American open habitats (Cerrado, Caatinga and Chaco); T - typical Cerrado species marginally occurring in adjoining domains; $\mathrm{W}$ - widespread species.

\begin{tabular}{|c|c|c|c|c|c|}
\hline TÁXON & Habitat & $\begin{array}{l}\text { Corpo } \\
\text { d'água }\end{array}$ & Distribuição & PEJ & EESGT \\
\hline \multicolumn{6}{|l|}{ ANURA } \\
\hline \multicolumn{6}{|l|}{ Brachycephalidae } \\
\hline Barycholos ternetzi Caramaschi \& & & - & & & \\
\hline Pombal, 2001 & $\mathrm{~F}$ & & $\mathrm{E}$ & & $\mathrm{x}$ \\
\hline \multicolumn{6}{|l|}{ Bufonidae } \\
\hline Rhaebo guttatus (Schneider, 1799) & $\mathrm{F}$ & $\mathrm{C}$ & $\mathrm{AM}$ & $\mathrm{x}$ & $\mathrm{x}$ \\
\hline $\begin{array}{l}\text { Rhinella mirandaribeiroi (Gallardo, } \\
\text { 1965) }\end{array}$ & A & PT & $\mathrm{T}$ & $\mathrm{x}^{*}$ & $\mathrm{x}$ \\
\hline Rhinella ocellata (Günther, 1858) & A & PT & $\mathrm{T}$ & $\mathrm{x}$ & $\mathrm{x}$ \\
\hline \multicolumn{6}{|l|}{ Cycloramphidae } \\
\hline $\begin{array}{l}\text { Proceratophrys goyana (Miranda- } \\
\text { Ribeiro, 1937) }\end{array}$ & $\mathrm{A} / \mathrm{F}$ & $\mathrm{C}$ & E & $\mathrm{X}^{*}$ & $\mathrm{x}$ \\
\hline \multicolumn{6}{|l|}{ Hylidae } \\
\hline Corythomantis greeningi & & PT & & & \\
\hline Boulenger, 1896 & A & & $\mathrm{CA}$ & & $\mathrm{x}$ \\
\hline Dendropsophus cruzi (Pombal e & & M & & & \\
\hline Bastos, 1998) & $\mathrm{F}$ & & $\mathrm{E}$ & $x^{*}$ & $\mathrm{x}$ \\
\hline $\begin{array}{l}\text { Dendropsophus minutus (Peters, } \\
\text { 1872) }\end{array}$ & A & PT & W & $\mathrm{x}$ & $\mathrm{x}$ \\
\hline
\end{tabular}




\begin{tabular}{|c|c|c|c|c|c|}
\hline TÁXON & Habitat & $\begin{array}{l}\text { Corpo } \\
\text { d'água }\end{array}$ & Distribuição & PEJ & EESGT \\
\hline Dendropsophus rubicundulus & & PT & & $\mathrm{x}$ & \\
\hline (Reinhardt \& Lütken, 1862) & A & & $\mathrm{T}$ & & $\mathrm{x}$ \\
\hline Dendropsophus soaresi & & PT & & & \\
\hline (Caramaschi e Jim, 1983) & $\mathrm{F}$ & & CA & & $\mathrm{x}$ \\
\hline $\begin{array}{l}\text { Hypsiboas albopunctatus (Spix, } \\
1824 \text { ) }\end{array}$ & $\mathrm{A} / \mathrm{F}$ & PT & W & $\mathrm{x}$ & $\mathrm{x}$ \\
\hline $\begin{array}{l}\text { Hypsiboas punctatus (Schneider, } \\
1799 \text { ) }\end{array}$ & $\mathrm{F}$ & M & W & $\mathrm{x}$ & $\mathrm{x}$ \\
\hline Hypsiboas raniceps Cope, 1862 & A & PT & W & $\mathrm{x}$ & $\mathrm{x}$ \\
\hline Osteocephalus cf. taurinus & $\mathrm{F}$ & M & $\mathrm{E}$ & $\mathrm{x}^{*}$ & \\
\hline Phyllomedusa azurea Cope, 1862 & $\mathrm{~A} / \mathrm{F}$ & $\mathrm{PT} / \mathrm{M}$ & $\mathrm{T}$ & & $\mathrm{x}$ \\
\hline Scinax constrictus (Lima, Bastos \& & & M & & & \\
\hline Giaretta, 2005) & $\mathrm{F}$ & & $\mathrm{E}$ & $x^{*}$ & $\mathrm{x}$ \\
\hline Scinax fuscomarginatus (Lutz, & & PT & & & \\
\hline 1925$)$ & A & & $\mathrm{W}$ & $\mathrm{x}$ & $\mathrm{x}$ \\
\hline Scinax fuscovarius (Lutz, 1925) & A & PT & W & $\mathrm{x}$ & \\
\hline Scinax sp. gr. ruber & A & PT & $\mathrm{E}$ & $x^{*}$ & $\mathrm{x}$ \\
\hline Trachycephalus venulosus & & PT & & & \\
\hline (Laurenti, 1768) & A & & $\mathrm{W}$ & $\mathrm{x}$ & \\
\hline Leiuperidae & & & & & \\
\hline $\begin{array}{l}\text { Eupemphix nattereri (Steindachner, } \\
1863 \text { ) }\end{array}$ & A & PT & $E$ & & $\mathrm{x}$ \\
\hline Physalaemus centralis Bokermann, & & PT & & & \\
\hline 1962 & A & & $\mathrm{T}$ & $\mathrm{x}$ & $\mathrm{x}$ \\
\hline Physalaemus cuvieri Fitzinger, & & PT & & & \\
\hline 1826 & A & & $\mathrm{W}$ & $\mathrm{x}$ & $\mathrm{x}$ \\
\hline Pleurodema diplolistris (Peters, & & PT & & & \\
\hline 1870) & A & & CA & $\mathrm{x}$ & $\mathrm{x}$ \\
\hline Pseudopaludicola mystacalis & & PT & & & \\
\hline (Cope, 1887) & A & & $\mathrm{W}$ & $\mathrm{x}$ & $\mathrm{x}$ \\
\hline Pseudopaludicola saltica (Cope, & & $\mathrm{C}$ & & & \\
\hline 1887) & A & & $\mathrm{E}$ & $\mathrm{x}$ & $\mathrm{x}$ \\
\hline Leptodactylidae & & & & & \\
\hline Leptodactylus fuscus (Schneider, & & PT & & & \\
\hline 1799) & A & & $\mathrm{W}$ & $\mathrm{x}$ & $\mathrm{x}$ \\
\hline Leptodactylus hylaedactylus & & - & & & \\
\hline Müller, 1923 & $\mathrm{~F}$ & & W & $\mathrm{x}^{*}$ & $\mathrm{x}$ \\
\hline Leptodactylus labyrinthicus (Spix, & & PT & & & \\
\hline 1824$)$ & A & & $\mathrm{W}$ & $\mathrm{x}$ & $\mathrm{x}$ \\
\hline Leptodactylus martinezi & & UM & & & \\
\hline Bokermann, 1956 & A & & $\mathrm{W}$ & $\mathrm{x}$ & $\mathrm{x}$ \\
\hline Leptodactylus latrans (Steffen, & & PT & & & \\
\hline 1815$)$ & A & & W & $\mathrm{x}$ & $\mathrm{x}$ \\
\hline Leptodactylus podicipinus (Cope, & & PT & & & \\
\hline 1862$)$ & A & & $\mathrm{O}$ & & $\mathrm{x}$ \\
\hline Leptodactylus sertanejo (Giaretta \& & & UM & & & \\
\hline Costa, 2007) & A & & $\mathrm{E}$ & $x^{*}$ & $\mathrm{x}$ \\
\hline Leptodactylus syphax Bokermann, & A & M & $\mathrm{T}$ & & $\mathrm{x}$ \\
\hline
\end{tabular}




\begin{tabular}{|c|c|c|c|c|c|}
\hline TÁXON & Habitat & $\begin{array}{l}\text { Corpo } \\
\text { d'água }\end{array}$ & Distribuição & PEJ & EESGT \\
\hline 1969 & & & & & \\
\hline $\begin{array}{l}\text { Leptodactylus troglodytes Lutz, } \\
1926\end{array}$ & A & PT & CA & $\mathrm{x}$ & $\mathrm{x}$ \\
\hline Microhylidae & & & & & \\
\hline $\begin{array}{l}\text { Dermatonotus muelleri (Boettger, } \\
1885 \text { ) }\end{array}$ & $\mathrm{A}$ & PT & $\mathrm{O}$ & $\mathrm{x}$ & $\mathrm{x}$ \\
\hline $\begin{array}{l}\text { Elachistocleis cesarii (Miranda- } \\
\text { Ribeiro, 1920) }\end{array}$ & $\mathrm{A}$ & PT & $\mathrm{W}$ & $\mathrm{x}$ & $\mathrm{x}$ \\
\hline $\begin{array}{l}\text { APODA } \\
\text { Caecilidae }\end{array}$ & & & & & \\
\hline Siphonops paulensis Boettger, 1892 & A & UM & W & $\mathrm{x}$ & $\mathrm{x}$ \\
\hline
\end{tabular}

Tabela 2. Riqueza total de espécies de anfíbios e riqueza das famílias com maior número de espécies de áreas de Cerrado amostradas por nosso estudo e estudos anteriores.

Table 2. Amphibians species richness and richness of the four richest families in cerrado sites sampled by us and presented by former studies.

\begin{tabular}{|c|c|c|c|c|c|}
\hline Local & Hylidae & Leptodactyidae & Leiuperidae & Bufonidae & Total \\
\hline $\begin{array}{l}\text { EESGT, BA / } \\
\text { TO }\end{array}$ & $12(0,33)$ & $9(0,25)$ & $6(0,17)$ & $4(0,11)$ & 36 \\
\hline PEJ, TO & $12(0,37)$ & $6(0,19)$ & $6(0,19)$ & $4(0,12)$ & 32 \\
\hline Palmas, TO & $22(0,42)$ & $12(0,23)$ & $4(0,07)$ & $5(0,09)$ & 52 \\
\hline $\begin{array}{l}\text { Vale do } \\
\text { Tocantins, TO }\end{array}$ & $15(0,45)$ & $8(0,24)$ & $4(0,12)$ & $3(0,09)$ & 33 \\
\hline $\begin{array}{l}\text { Vale do } \\
\text { Tocantins, MA }\end{array}$ & $16(0,48)$ & $7(0,21)$ & $4(0,12)$ & $3(0,09)$ & 33 \\
\hline $\begin{array}{l}\text { São Desidério, } \\
\text { BA }\end{array}$ & $10(0,31)$ & $8(0,25)$ & $7(0,22)$ & $5(0,16)$ & 32 \\
\hline Jataí, GO & $16(0,50)$ & $4(0,12)$ & $5(0,16)$ & $3(0,09)$ & 32 \\
\hline $\begin{array}{l}\text { Mambaí, GO } \\
\text { Manso, MT }\end{array}$ & $14(0,46)$ & $4(0,13)$ & $5(0,17)$ & $2(0,07)$ & 30 \\
\hline Itiquira, MT & $15(0,45)$ & $8(0,24)$ & $6(0,18)$ & $1(0,03)$ & 33 \\
\hline Bodoquena, MS & $16(0,42)$ & $4(0,10)$ & $8(0,21)$ & $4(0,10)$ & 38 \\
\hline Itirapina, SP & $13(0,46)$ & $6(0,21)$ & $4(0,14)$ & $2(0,07)$ & 28 \\
\hline Pedregulho, SP & $10(0,41)$ & $3(0,12)$ & $4(0,17)$ & $2(0,08)$ & 24 \\
\hline
\end{tabular}

Fonte: EESGT, BA / TO - Este estudo; PEJ - Vitt et al. (2005); Itirapina, SP -

Brasileiro et al. (2005) Pedregulho, SP - Araujo et al. (2009); São Desidério, BA Valdujo et al. (2009); Bodoquena, MS - Uetanabaro et al. (2007); Jataí, GO - VazSilva et al. (2007); Vale do Tocantins, MA - Brasileiro et al. (2007); Vale do Tocantins, TO - Brasileiro et al (2007); Palmas, TO - Pavan e Dixo (2004); Mambaí, GO - Cintra et al (2009); Itiquira, MT - Silva-Jr et al (2009). 


\section{Legendas das Figuras}

Figura 1. Mapa da Estação Ecológica Serra Geral de Tocantins (contorno amarelo), indicando os rios da região e os pontos de amostragem de anfíbios. Os círculos pretos representam pontos amostrados por meio de procura visual e os círculos brancos indicam pontos amostrados por armadilhas de queda.

Figure 1. Map of Estação Ecológia Serra Geral de Tocantins (yellow line), with rivers and sampling sites. Black circles represent sites sampled by visual search and white circules are sites sampled by pitfall traps.

Figura 2. Curva de rarefação construídas a partir de 10.000 aleatorizações dos valores de riqueza de espécies de anfíbios amostradas a cada dia de amostragem na EESGT. Símbolos pretos representam aleatorizações dos valores observados e símbolos brancos representam valores de riqueza calculados a partir do estimador Jackknife; as barras representam o desvio padrão das aleatorizações.

Figure 2. Sample-based rarefaction curve after 10.000 randomizations of amphibian species richness values. Black circles represent the results of randomization of observed data and hollow circles represent Jackknife estimator; bars represent randomization standard deviations.

Figura 3: Anfíbios da Estação Ecológica Serra Geral do Tocantins: A Dendropsophus cruzi; B - D. minutus; C - D. rubicundulus; D - D. soaresi; E Hypsiboas punctatus; F - H. albopunctatus; G - Scinax constrictus; H - Phyllomedusa azurea. Fotos: P.H. Valdujo

Figure 3: Amphibians from Estação Ecológica Serra Geral do Tocantins: A Dendropsophus cruzi; B - D. minutus; C - D. rubicundulus; D - D. melanargyreus; E Hypsiboas punctatus; F - H. albopunctatus; G - Scinax constrictus; H - Phyllomedusa azurea. Fotos: P.H. Valdujo

Figura 4: Anfíbios da Estação Ecológica Serra Geral do Tocantins. A Corythomantis greeningi; B - Pleurodema diplolister; C - Physalaemus cuvieri; D - P. centralis; E - Pseudopaludicola mystacalis; F - P. saltica; G - Eupemphix nattereri; H - Proceratophrys goyana. Fotos: P.H. Valdujo.

Figure 4: Amphibians from Estação Ecológica Serra Geral do Tocantins. A Corythomantis greeningi; B - Pleurodema diplolister; C - Physalaemus cuvieri; D - P. centralis; E - Pseudopaludicola mystacalis; F - P. saltica; G - Eupemphix nattereri; H - Proceratophrys sp. Fotos: P.H. Valdujo.

Figura 5: Anfíbios da Estação Ecológica Serra Geral do Tocantins. A - Leptodactylus podicipinus; B - L. troglodytes; C - L. hylaedactylus; D - L. fuscus; E - L. martinezi; F - L. sertanejo; G - L. syphax; H - L. labyrinthicus. Fotos: P.H. Valdujo.

Figure 5: Amphibians from Estação Ecológica Serra Geral do Tocantins. A Leptodactylus podicipinus; B - L. troglodytes; C - L. hylaedactylus; D - L. fuscus; E L. martinezi; F - L. sertanejo; G - L. syphax; H - L. labyrinthicus. Fotos: P.H. Valdujo. 
Figura 6: Anfíbios da Estação Ecológica Serra Geral do Tocantins. A - Rhinella mirandaribeiroi; B - R. schneideri; C - Rhaebo guttatus; D - Rhnella ocellata; E Elachistocleis cesarii; F - Barycholos ternetzi; G - Dermatonotus muelleri; H Siphonops paulensis. Fotos: A - F e H: P.H. Valdujo; foto G: M. TeixeiraJr.

Figure 6: Amphibians from Estação Ecológica Serra Geral do Tocantins. A - Rhinella mirandaribeiroi; B - R. schneideri; C - Rhaebo guttatus; D - Rhnella ocellata; E Elachistocleis cesarii; F - Barycholos ternetzi; G - Dermatonotus muelleri; H Siphonops paulensis. Fotos: A - F e H: P.H. Valdujo; foto G: M. Teixeira Jr.

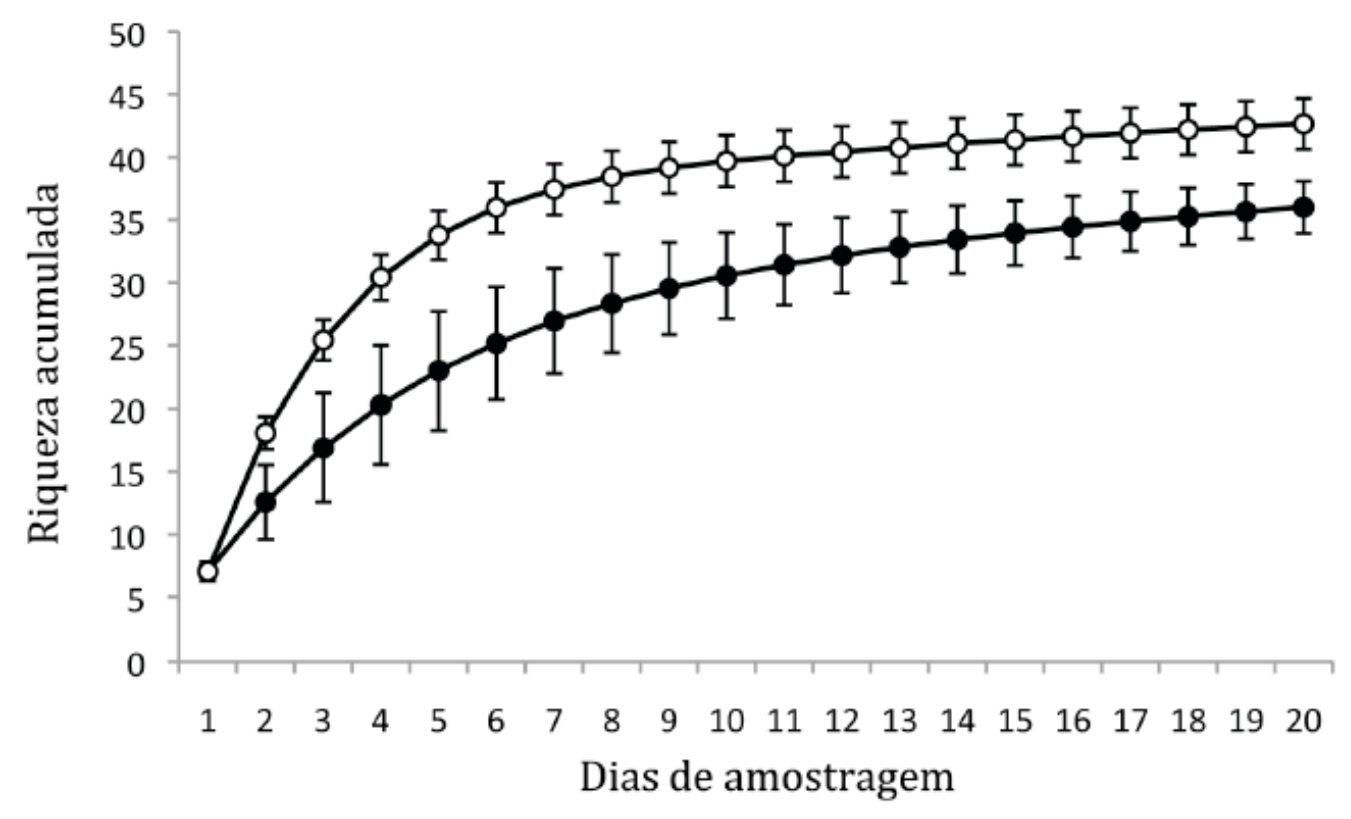




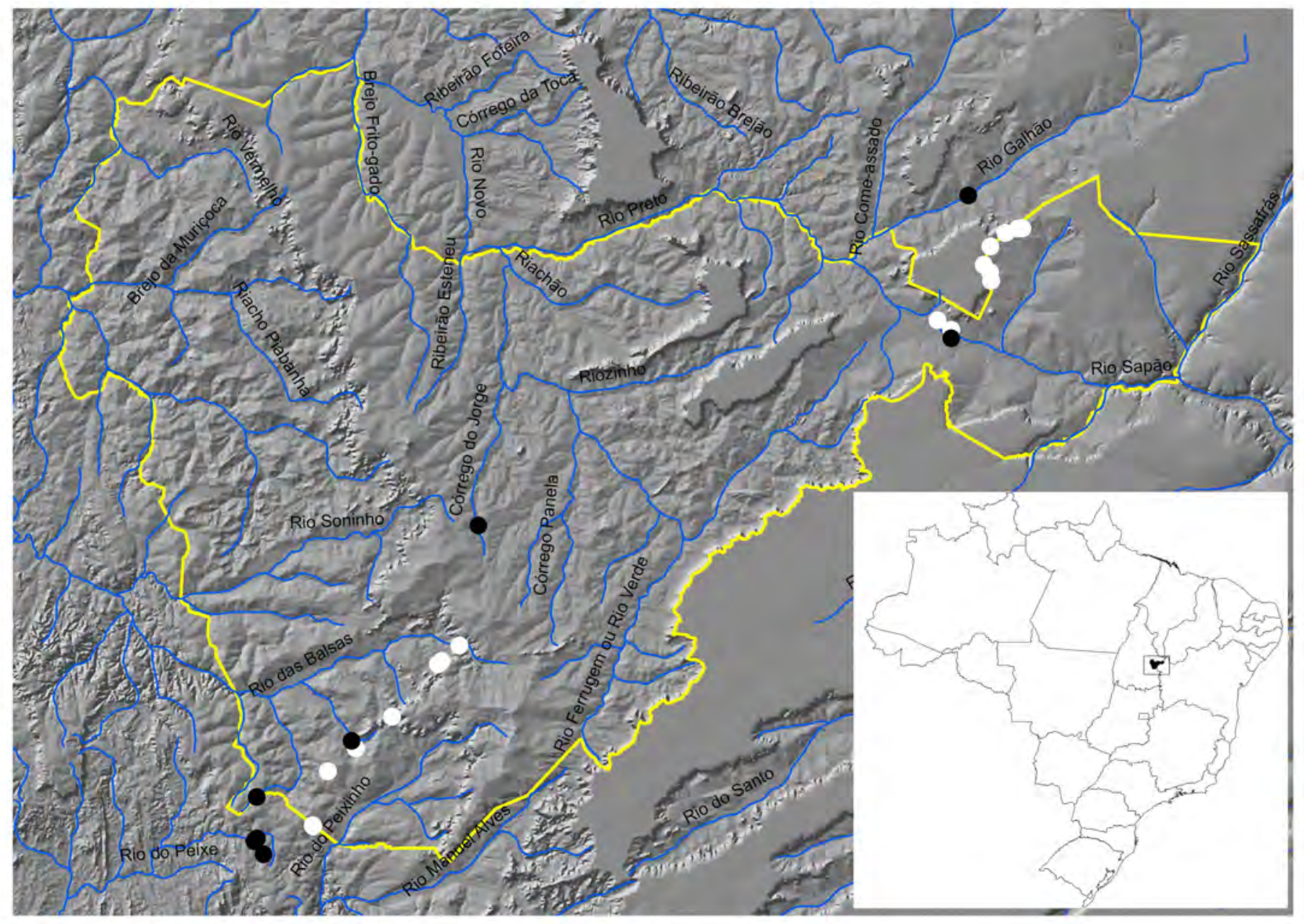



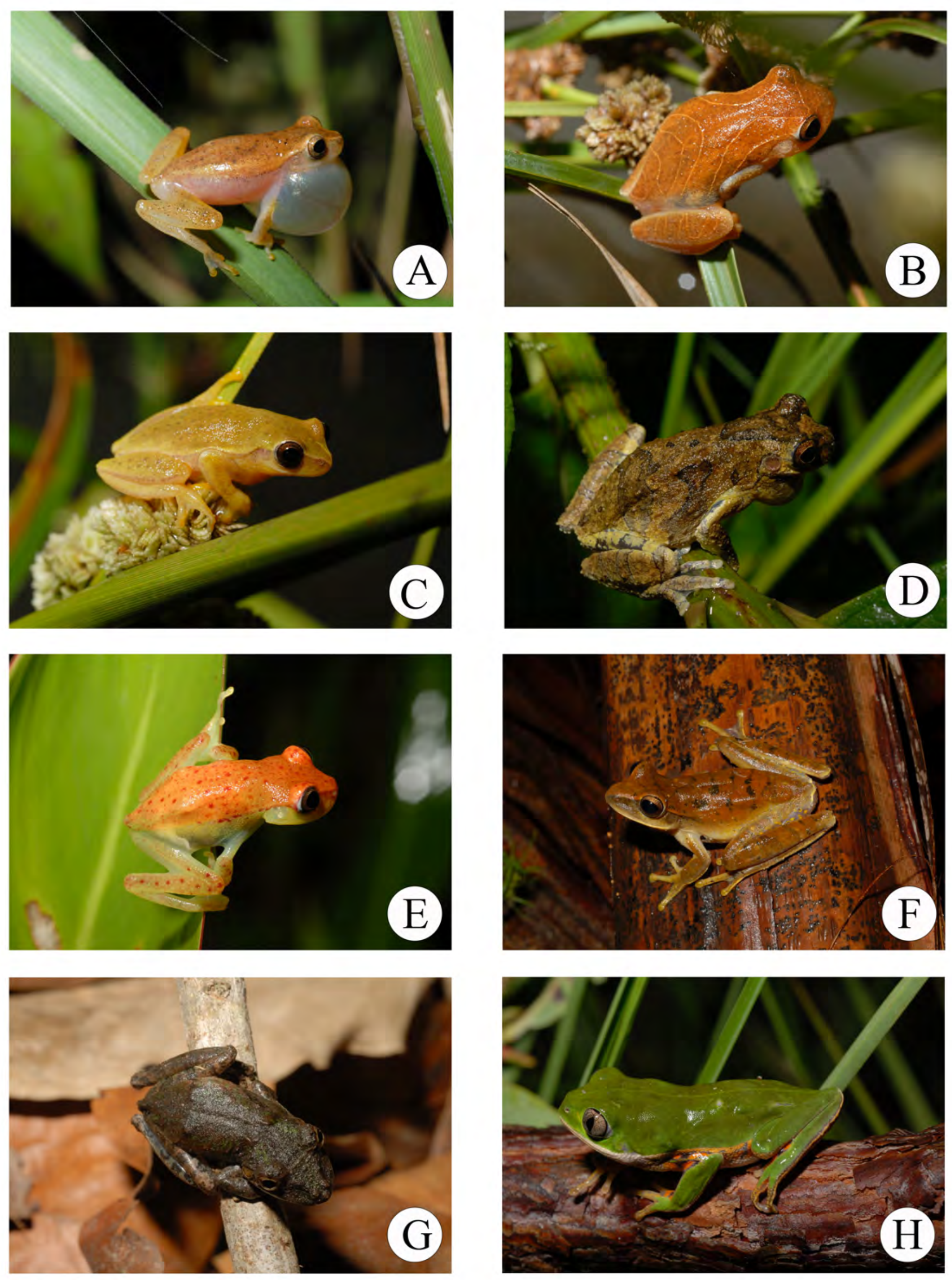

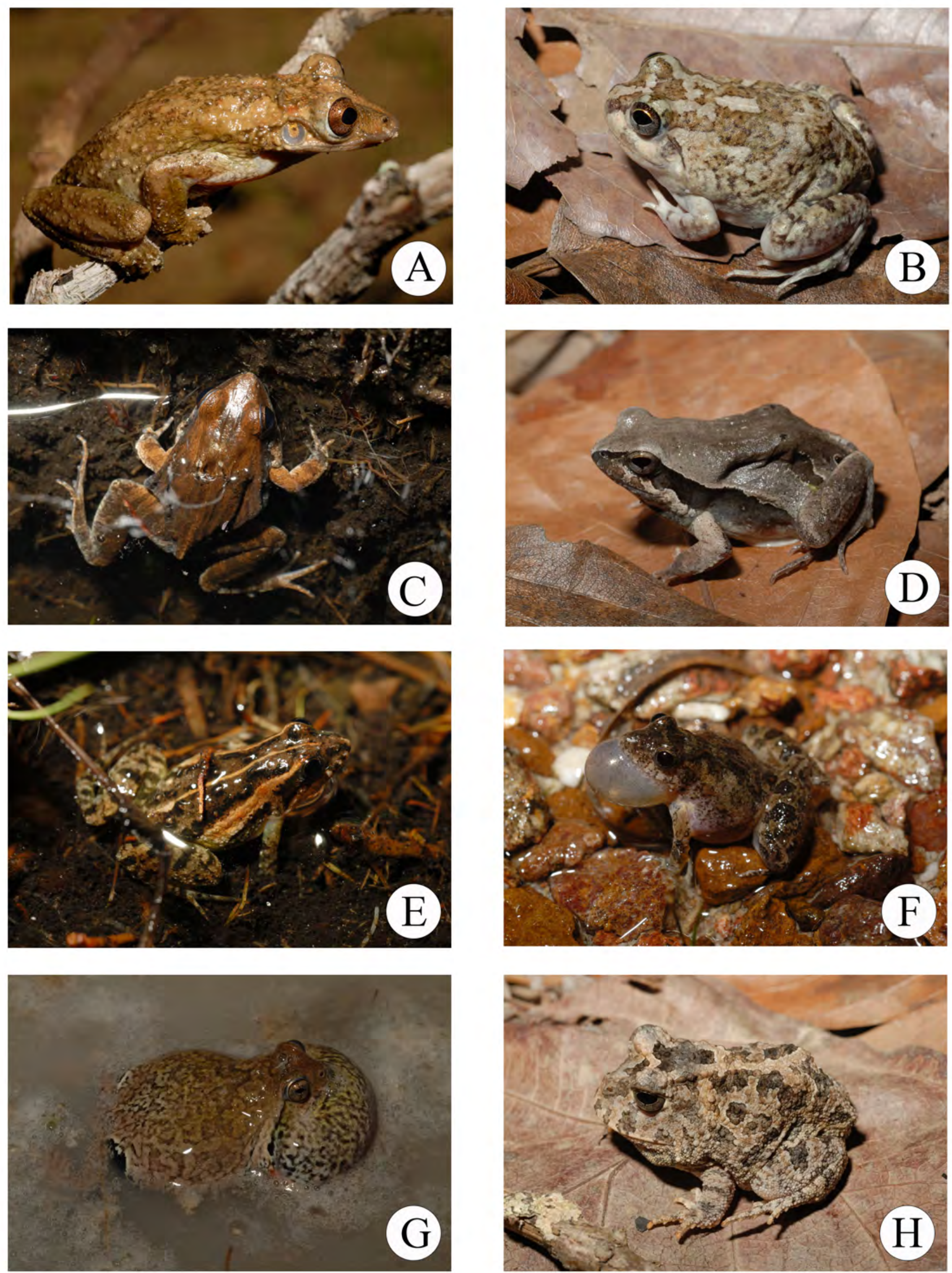

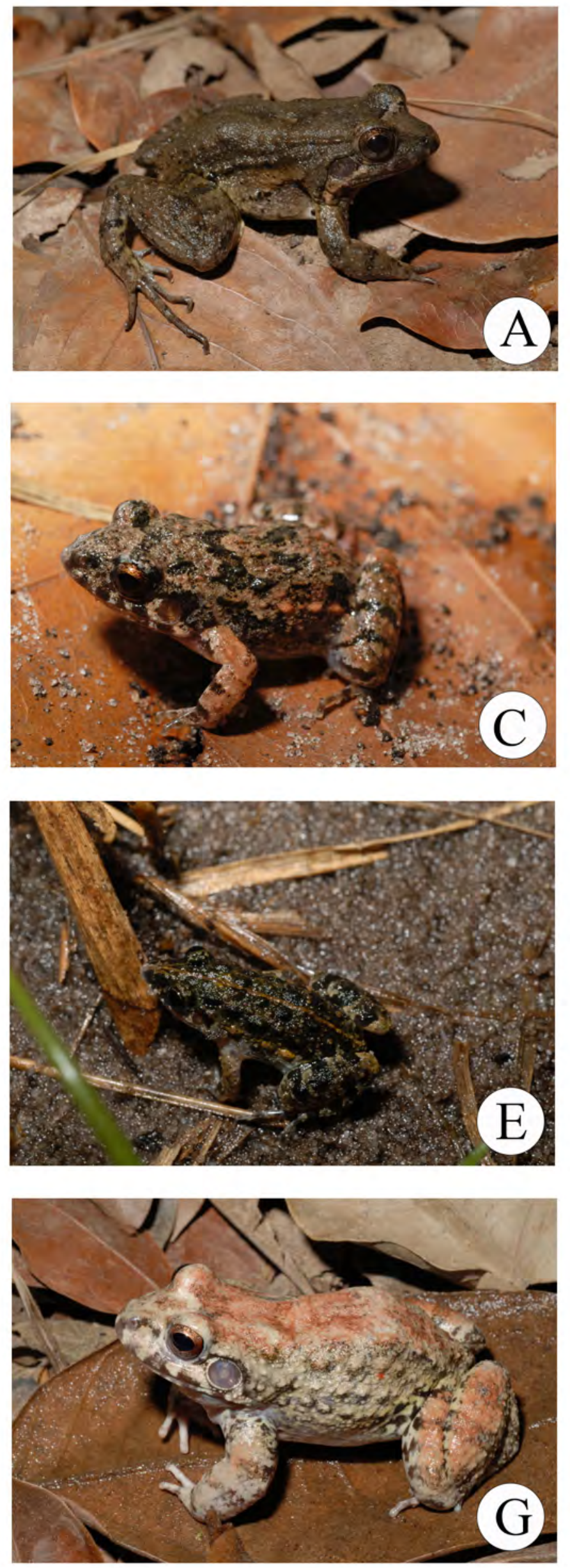
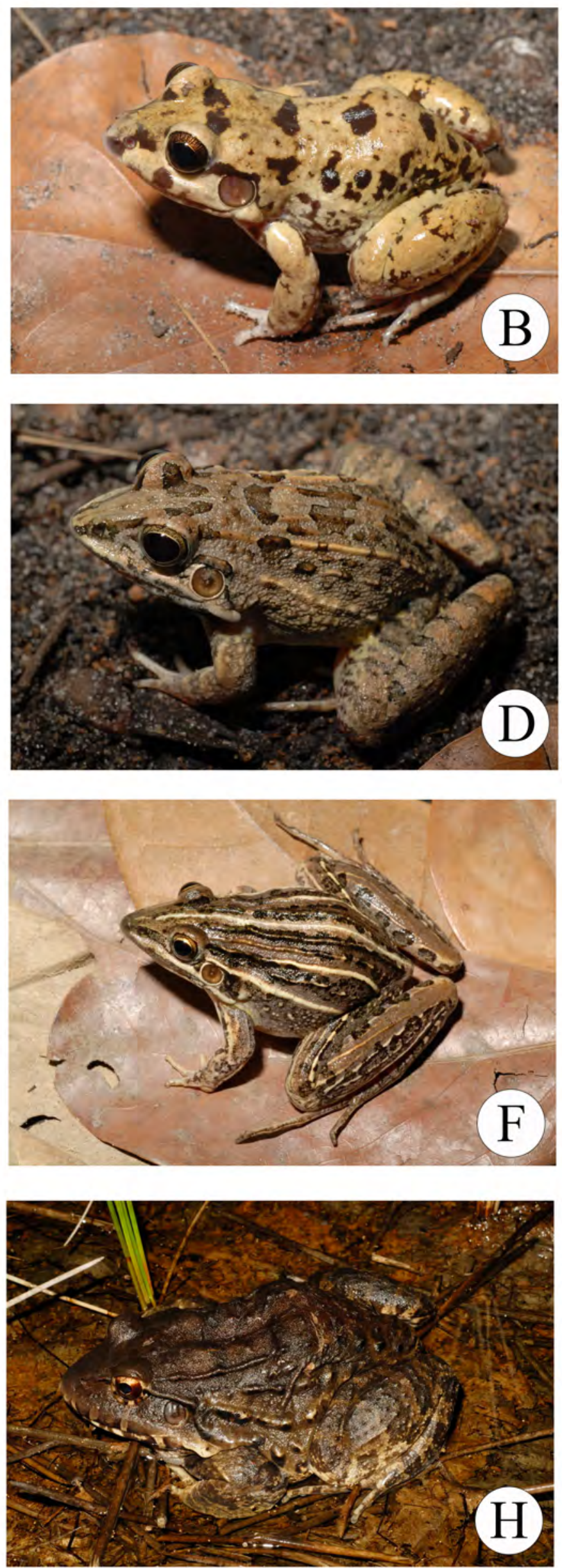

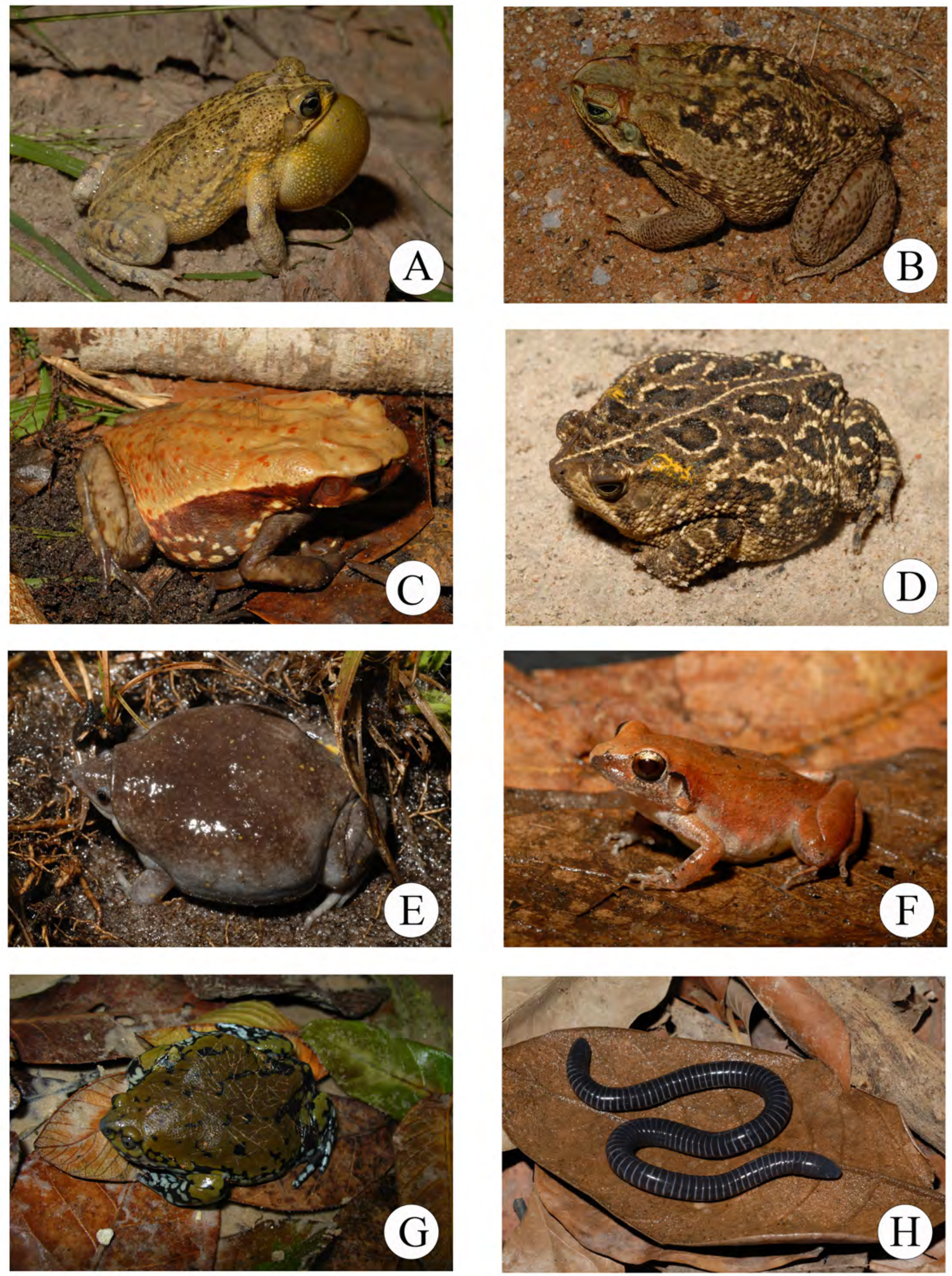